\title{
On generalized Newtonian fluids in curved pipes*
}

\author{
Nadir Arada ${ }^{\dagger}$
}

\begin{abstract}
This paper is concerned with steady, fully developed motion of a Navier-Stokes fluid with shear-dependent viscosity in a curved pipe under a given axial pressure gradient. We establish existence and uniqueness results, derive appropriate estimates and prove a characterization of the secondary flows. The approximation, with respect to the curvature ratio, of the full governing systems by some Dean-like equation is studied.
\end{abstract}

Key words. Navier-Stokes fluids, shear-dependent viscosity, shear-thinning flows, shearthickening flows, curved pipes.

AMS Subject Classification. 35J65, 76A05, 76D03.

\section{Introduction}

There is a great interest in the study of curved pipe flows due to its wide range of applications in engineering (e.g. hydraulic pipe systems related to corrosion failure) and in biofluid dynamics, such as blood flow in the vascular system. It is known since the pioneer experimental works of Eustice $(7],[8])$ that these flows are challenging and much more complex than flows in straight pipes. Among their distinguishing features is the existence of secondary flows induced by the centrifugal force and which appear even for the slightest curvature.

Fully developed viscous flow in a curved pipe with circular cross-section was first studied theoretically by Dean $([5,6]$ ) in the case of Newtonian fluids by applying regular perturbation methods, the perturbation parameter being the curvature ratio. He simplified the governing equations, by neglecting all the effects due to pipe curvature except the centrifugal forces, and showed that for small curvature ratio the flow depends only on a single parameter, the so-called Dean number. Following this fundamental work, the results based on perturbation solutions have been extended for a larger range of curvature ratio and Reynolds number, showing in particular the existence of additional pairs of vortices and multiple solutions (see e.g. [23, [4]). Different geometries including circular, elliptical and annular cross-sections have also been considered by several authors (see e.g. [2, [12], [19, 21], 22]).

Despite the great interest in curved pipes, a rigorous analysis of the solvability of the Dean's equations and the full Navier-Stokes equations was not available prior to the work of Galdi and Robertson [10, where existence and uniqueness results for steady, fully developed flows are established. Existence of secondary motions is also studied, as well as the approximation of the Navier-Stokes equations by the Dean's equation for small curvature ratios.

\footnotetext{
*This work was partially supported by FCT project PEst-OE/MAT/UI4032/2011

${ }^{\dagger}$ Departamento de Matemática, Faculdade de Ciências e Tecnologia da Universidade Nova de Lisboa, Quinta da Torre, 2829-516 Caparica, Portugal (naar@fct.unl.pt).
} 
Flows of non-Newtonian fluids in curved pipes have also been studied by several authors (see e.g. 3, 9, 213, 20]). Perturbation methods were used by Robertson and Muller 20, to study steady, fully developed flow of Oldroyd-B fluids, and to compare the results for creeping and non-creeping flows. For a second order model, Jitchote and Robertson [13] obtained analytical solutions to the perturbation equations and analyze the effects of non-zero second normal stress coefficient on the behaviour of the solution. Theoretical results regarding this problem were obtained by Coscia and Robertson in [3], where existence and uniqueness of a strong solution for small non-dimensional pressure drop is established.

The aim of the present paper is to extend the analysis carried-out in [10 to the class of quasiNewtonian fluids. This class is described by partial differential equations of the quasilinear type (Navier-Stokes equations with a non constant viscosity that decreases with increasing shear rate in the case of shear-thinning flows and increases with increasing shear rate in the case of shearthickening flows). It was first proposed and studied in bounded domains by Ladyzhenskaya in [14], [15] and [16] as a modification of the Navier-Stokes system, and was similarly suggested by Lions in [17. Existence of weak solutions was proved by both authors using compactness arguments and the theory of monotone operators. Much work has been done since these pioneering results and, without ambition for completeness, we cite Nečas et al. who established existence of weak solutions under less restrictive assumptions (see for example [18).

Since we are dealing with fully developed flows in curved pipes, the typical issues related to the nonlinear extra stress tensor and the convective term arise and can be handled as in the case of bounded domains. However, additional difficulties related with extra terms (depending on the curvature ratio) occur and need to be managed. The splitting method, consisting in two coupled formulations respectively associated to the secondary flows and to the axial flow and used in 10 for the study of the Newtonian case, cannot be applied. Because of the nonlinearity of the shear stress tensor, the coercivity property of the corresponding bilinear forms in the shear-thinning case, and the monotonicity property of these forms in both shear-thinning and shear thickening cases fail to be satisfied. To overcome these difficulties, we consider a global formulation in an appropriate functional setting and adapt some standard tools, such as the Korn inequality. An existence result is established for arbitrary values of the Reynolds number, of the pressure drop and for any curvature ratio of the pipe, and a uniqueness result for small Reynolds numbers. The global formulation allows also to derive uniform estimates independent of the Dean number. Using a posteriori the splitting method, we establish other estimates for the secondary flows that highlight the connection with the Dean number. Following [10], we also prove that there are no secondary flows if, and only if, the Dean number is equal to zero. Finally, we consider an approximation problem (that can be seen as a generalization of the Dean's equation), study its solvability, establish corresponding estimates and evaluate the relationship between its solutions and those of the full governing equations.

The plan of the paper is as follows. The governing equations are given in Section 2. Notation, and some preliminary results are given in Section 3. Section 4 is devoted to the statement and discussion of the main results. In Section 5, we consider the case of the shear-thickening flows. We establish a version of the Korn inequality more appropriate for our framework and derive some estimates for the convective term and the extra stress tensor. The existence and uniqueness results for the full governing equations are then established and the approximation analysis is carried out. The shear-thinning case is treated in a similar way in Section 6 . 


\section{Governing Equations}

We are concerned with steady flows of incompressible generalized Newtonian fluids. For these fluids, the Cauchy stress tensor $\widetilde{\boldsymbol{T}}$ is related to the kinematic variables through

$$
\widetilde{\boldsymbol{T}}=-\tilde{\pi} I+2 \mu\left(1+|\widetilde{D} \widetilde{\boldsymbol{u}}|^{2}\right)^{\frac{p-2}{2}} \widetilde{D} \widetilde{\boldsymbol{u}}
$$

where $\widetilde{\boldsymbol{u}}$ is the velocity field, $\widetilde{D} \widetilde{\boldsymbol{u}}=\frac{1}{2}\left(\widetilde{\nabla} \widetilde{\boldsymbol{u}}+\widetilde{\nabla} \widetilde{\boldsymbol{u}}^{\top}\right)$ denotes the symmetric part of the velocity gradient, $\mu>0$ is the kinematic viscosity, and $\widetilde{\pi}$ represents the pressure. The notation $\sim$ denotes a dimensional quantity. The equations of conservation of momentum and mass, relative to a rectangular coordinate system, are

$$
\left\{\begin{array}{l}
\rho\left(\frac{\partial \widetilde{\boldsymbol{u}}}{\partial \widetilde{t}}+\widetilde{\boldsymbol{u}} \cdot \widetilde{\nabla} \widetilde{\boldsymbol{u}}\right)+\widetilde{\nabla} \widetilde{\pi}=\widetilde{\nabla} \cdot\left(2 \mu\left(1+|\widetilde{D} \widetilde{\boldsymbol{u}}|^{2}\right)^{\frac{p-2}{2}} \widetilde{D} \widetilde{\boldsymbol{u}}\right), \\
\widetilde{\nabla} \cdot \widetilde{\boldsymbol{u}}=0
\end{array}\right.
$$

where $\rho>0$ is the constant density of the fluid. In this work, we consider steady flow of generalized Newtonian fluids through a curved pipe of arbitrary shaped cross-section $\Sigma$ with constant centerline radius $R$. Due to the geometric characteristics of the curved pipe, it is convenient to write system (2.1) in the rectangular toroidal coordinates $\left(\widetilde{x}_{i}\right)$ defined with respect to the rectangular Cartesian coordinates $\left(\widetilde{y}_{i}\right)$ through the relations

$$
\widetilde{x}_{1}=\widetilde{y}_{3}, \quad \widetilde{x}_{2}=\sqrt{\widetilde{y}_{1}^{2}+\widetilde{y}_{2}^{2}}-R, \quad \widetilde{x}_{3}=R \arctan \frac{\widetilde{y}_{2}}{\widetilde{y}_{1}}
$$

and inverse relations

$$
\widetilde{y}_{1}=\left(R+\widetilde{x}_{2}\right) \cos \left(\frac{\widetilde{x}_{3}}{R}\right), \quad \widetilde{y}_{2}=\left(R+\widetilde{x}_{2}\right) \sin \left(\frac{\widetilde{x}_{3}}{R}\right), \quad \widetilde{y}_{3}=\widetilde{x}_{1} .
$$

More details on the toroidal coordinate system, and on the corresponding formulation of the operators involved in (2.1), can be found in Appendix A.

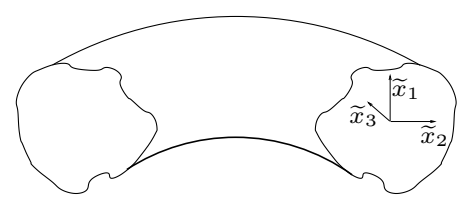

Figure 1: Rectangular toroidal coordinates in a curved pipe

We restrict our study to curved pipes with cross section independent of $\tilde{x}_{3}$, and consider flows which are steady and fully developed, i.e. the components of the velocity vector with respect to the new basis are independent of both time and axial variable $x_{3}$. For such flows

$$
\frac{\partial \widetilde{u}_{i}}{\partial \widetilde{x}_{3}}=0 \quad i=1,2,3
$$

and the axial component of the pressure gradient

$$
\frac{\partial \widetilde{\pi}}{\partial \widetilde{x}_{3}}=-\widetilde{G}
$$


is a constant. We consider the non-dimensional form of this system by introducing the following quantities

$$
r_{0}=\sup _{\widetilde{x} \in \bar{\Sigma}}|\widetilde{x}|, \quad x_{i}=\frac{\widetilde{x}_{i}}{r_{0}}, \quad \boldsymbol{u}=\frac{\widetilde{u}}{U_{0}}, \quad \pi=\frac{\widetilde{\pi} r_{0}}{\mu U_{0}}, \quad G=\frac{\widetilde{G} r_{0}^{2}}{\mu U_{0}},
$$

where $\delta \in\left[0,1\left[\right.\right.$ is the pipe curvature ratio, and $U_{0}$ represents a characteristic velocity of the flow. We also introduce the Reynolds number $\mathcal{R} e=\frac{\rho U_{0} r_{0}}{\mu}$ and the pipe curvature ratio $\delta=\frac{r_{0}}{R} \in[0,1[$. The governing equations can then be written with respect to the non-dimensional quantities as

$$
\begin{cases}-\nabla^{\star} \cdot\left(\boldsymbol{\tau}\left(D^{\star} \boldsymbol{u}\right)\right)+\mathcal{R} e \boldsymbol{u} \cdot \nabla^{\star} \boldsymbol{u}+\nabla^{\star} \pi=0 & \text { in } \Sigma, \\ \nabla^{\star} \cdot \boldsymbol{u}=0 & \text { in } \Sigma, \\ \boldsymbol{u}=0 & \text { on } \partial \Sigma\end{cases}
$$

with

$$
\boldsymbol{\tau}\left(D^{\star} \boldsymbol{u}\right)=2\left(1+\dot{\gamma}^{2}\left|D^{\star} \boldsymbol{u}\right|^{2}\right)^{\frac{p-2}{2}} D^{\star} \boldsymbol{u}
$$

where $\dot{\gamma}=\frac{U_{0}}{r_{0}}$ is a caracteristic shear-rate. In order to simplify the redaction, we will assume without lost of generality that $\dot{\gamma}=1$. The operators involved in the definition of (2.7), are defined by

with

$$
\begin{aligned}
& \nabla^{\star} \cdot \boldsymbol{\tau}=\nabla \cdot \boldsymbol{\tau}+\frac{\delta}{B}\left(\tau_{12} \boldsymbol{a}_{1}+\left(\tau_{22}-\tau_{33}\right) \boldsymbol{a}_{2}+2 \tau_{23} \boldsymbol{a}_{3}\right), \\
& \nabla^{\star} \boldsymbol{u}=\nabla \boldsymbol{u}+\frac{\delta}{B}\left(u_{2} \boldsymbol{a}_{3} \otimes \boldsymbol{a}_{3}-u_{3} \boldsymbol{a}_{3} \otimes \boldsymbol{a}_{2}\right), \\
& D^{\star} \boldsymbol{u}=\frac{1}{2}\left(\nabla^{\star} \boldsymbol{u}+\nabla^{\star} \boldsymbol{u}^{\top}\right), \\
& \boldsymbol{u} \cdot \nabla^{\star} \boldsymbol{u}=\boldsymbol{u} \cdot \nabla \boldsymbol{u}+\frac{\delta}{B}\left(u_{3} u_{2} \boldsymbol{a}_{3}-u_{3}^{2} \boldsymbol{a}_{2}\right), \\
& \nabla^{\star} \pi=\nabla \pi-\frac{G}{B} \boldsymbol{a}_{3} \\
& \nabla^{\star} \cdot \boldsymbol{u}=\frac{\partial u_{1}}{\partial x_{1}}+\frac{\partial u_{2}}{\partial x_{2}}+\frac{\delta}{B} u_{2}=\frac{1}{B} \nabla \cdot(B \boldsymbol{u})
\end{aligned}
$$

$$
\nabla=\left(\frac{\partial}{\partial x_{1}}, \frac{\partial}{\partial x_{2}}, 0\right), \quad \nabla \cdot=\frac{\partial}{\partial x_{1}}+\frac{\partial}{\partial x_{2}}, \quad B=1+\delta x_{2},
$$

and where $\left(\boldsymbol{a}_{1}, \boldsymbol{a}_{2}, \boldsymbol{a}_{3}\right)$ denotes the orthonormal basis in the toroidal coordinates. (For a detailed derivation of the dimensionless equation (2.7), see Appendix B],

\section{Notation, assumptions and preliminary results}

\subsection{Algebraic results}

Throughout the paper, if $\boldsymbol{u}=\left(u_{1}, u_{2}, u_{3}\right)$ in the rectangular toroidal coordinates, we denote by $u$ the vector with toroidal components $\left(u_{1}, u_{2}, 0\right)$. Similarly, if $\boldsymbol{S}=\left(S_{i j}\right)_{i, j=1,2,3}$ is a tensor in $\mathbb{R}^{3 \times 3}$, we denote by $S$ the tensor in $\mathbb{R}^{2 \times 2}$ with toroidal components $S_{i j}, i=1,2$.

For $\eta, \zeta \in \mathbb{R}^{d \times d}$, we define the scalar product and the corresponding norm by

$$
\eta: \zeta=\sum_{i, j=1}^{d} \eta_{i j} \zeta_{i j} \quad \text { and } \quad|\eta|=(\eta: \eta)^{\frac{1}{2}}
$$

In the next two results, we state well known continuity, coercivity and monotonicity properties for $\boldsymbol{\tau}$. The corresponding proof is standard (see e.g. [18]). We first consider the case $p \geq 2$ corresponding to the shear-thickening flows. 
Lemma 3.1 Assume that $p \geq 2$ and let $\eta \in \mathbb{R}_{\mathrm{sym}}^{3 \times 3}$. Then the tensor $\boldsymbol{\tau}$ satisfies the following properties

Continuity.

$$
|\boldsymbol{\tau}(\eta)-\boldsymbol{\tau}(\zeta)| \leq(p-1)\left(1+|\eta|^{2}+|\zeta|^{2}\right)^{\frac{p-2}{2}}|\eta-\zeta|
$$

Coercivity.

$$
\boldsymbol{\tau}(\eta): \eta \geq 2|\eta|^{2}, \quad \boldsymbol{\tau}(\eta): \eta \geq 2|\eta|^{p},
$$

Monotonicity.

$$
\left\{\begin{array}{l}
(\boldsymbol{\tau}(\eta)-\boldsymbol{\tau}(\zeta)):(\eta-\zeta) \geq|\eta-\zeta|^{2} \\
(\boldsymbol{\tau}(\eta)-\boldsymbol{\tau}(\zeta)):(\eta-\zeta) \geq \frac{1}{2^{p-1}(p-1)}|\eta-\zeta|^{p}
\end{array}\right.
$$

Next we consider the case $1<p<2$ corresponding to the shear-thinning flows.

Lemma 3.2 Assume that $1<p<2$ and let $\eta \in \mathbb{R}_{\mathrm{sym}}^{3 \times 3}$. Then the tensor $\boldsymbol{\tau}$ satisfies the following properties

Continuity.

$$
|\boldsymbol{\tau}(\eta)-\boldsymbol{\tau}(\zeta)| \leq C_{p}|\eta-\zeta|^{p-1} \quad \text { with } C_{p}=1+2^{\frac{2-p}{2}}
$$

Coercivity.

$$
\boldsymbol{\tau}(\eta): \eta \geq 2\left(1+|\eta|^{2}\right)^{\frac{p-2}{2}}|\eta|^{2}
$$

Monotonicity.

$$
(\boldsymbol{\tau}(\eta)-\boldsymbol{\tau}(\zeta)):(\eta-\zeta) \geq 2(p-1)\left(1+|\eta|^{2}+|\zeta|^{2}\right)^{\frac{p-2}{2}}|\eta-\zeta|^{2}
$$

\subsection{Functional setting}

Throughout the paper $\Sigma$ is a bounded domain in $\mathbb{R}^{2}$, with a boundary $\partial \Sigma$. Even though several of our results are valid for an arbitrary bounded domain, we will assume without loss of generality that $\Sigma$ is of class $C^{2}$. By $\boldsymbol{W}^{k, p}(\Sigma)(k \in \mathbb{N}$ and $1<p<\infty)$, we denote the standard Sobolev spaces and we denote the associated norms by $\|\cdot\|_{k, p}$. We set $\boldsymbol{W}^{0, p}(\Sigma) \equiv \boldsymbol{L}^{p}(\Sigma),\|\cdot\|_{L^{p}} \equiv\|\cdot\|_{p}$, $\boldsymbol{L}_{0}^{p}(\Sigma)=\left\{\boldsymbol{u} \in L^{p}(\Sigma) \mid \int_{\Sigma} \boldsymbol{u}(x) d x=0\right\}$, and

$$
\|\cdot\|_{p, B}=\left\|B^{\frac{1}{p}} \cdot\right\|_{p}, \quad m=\left\|\frac{1}{B}\right\|_{\infty} \quad \text { and } \quad n=\|B\|_{\infty}
$$

The dual space of $\boldsymbol{W}_{0}^{1, p}(\Sigma)$ is denoted by $\boldsymbol{W}^{-1, p^{\prime}}(\Sigma)$, where $p^{\prime}=\frac{p}{p-1}$ is the dual exponent to $p$, its norm is denoted by $\|\cdot\|_{-1, p^{\prime}}$ and the duality pairing between these spaces by $\langle\cdot, \cdot\rangle$. We will also use the following notation

$$
\begin{gathered}
(\boldsymbol{u}, \boldsymbol{v})=\int_{\Sigma} \boldsymbol{u}(x) \cdot \boldsymbol{v}(x) d x, \quad \boldsymbol{u} \in L^{p}(\Sigma), \boldsymbol{v} \in \boldsymbol{L}^{p^{\prime}}(\Sigma) \\
(\eta, \zeta)=\int_{\Sigma} \eta(x): \zeta(x) d x, \quad \eta \in \boldsymbol{L}^{p}\left(\Sigma, \mathbb{R}^{3 \times 3}\right), \zeta \in \boldsymbol{L}^{p^{\prime}}\left(\Sigma, \mathbb{R}^{3 \times 3}\right) .
\end{gathered}
$$


The space of infinitely differentiable functions with compact support in $\Sigma$ will be denoted by $\mathcal{D}(\Sigma)$. In order to eliminate the pressure in the weak formulation of our equation, we will work in divergence-free spaces. Consider

$$
\begin{gathered}
\mathcal{V}=\{\boldsymbol{\varphi} \in \mathcal{D}(\Sigma) \mid \nabla \cdot \boldsymbol{\varphi}=0\}, \\
\mathcal{V}_{B}=\{\boldsymbol{\varphi} \in \mathcal{D}(\Sigma) \mid \nabla \cdot(B \boldsymbol{\varphi})=0\},
\end{gathered}
$$

and denote by $\boldsymbol{V}^{p}$ and $\boldsymbol{V}_{B}^{p}$ the closure of $\mathcal{V}$ and $\mathcal{V}_{B}$ in the $L^{p}$-norm of gradients, i.e.

$$
\begin{gathered}
\boldsymbol{V}^{p}=\left\{\boldsymbol{\varphi} \in \boldsymbol{W}_{0}^{1, p}(\Sigma) \mid \nabla \cdot \boldsymbol{\varphi}=0\right\}, \\
\boldsymbol{V}_{B}^{p}=\left\{\boldsymbol{\varphi} \in \boldsymbol{W}_{0}^{1, p}(\Sigma) \mid \nabla \cdot(B \boldsymbol{\varphi})=0\right\} .
\end{gathered}
$$

Next, we recall the Sobolev inequality.

Lemma 3.3 Let $r$ and $q$ be such that $r \geq q$ if $q \geq 2$ and $1<r \leq q^{*}=\frac{2 q}{2-q}$ if $1<q<2$. Then for all all $\boldsymbol{u} \in \boldsymbol{W}_{0}^{1, q}(\Sigma)$, we have

$$
\|\boldsymbol{u}\|_{r} \leq S_{q, r}\|\nabla \boldsymbol{u}\|_{q}
$$

where

$$
S_{q, r}= \begin{cases}\frac{\max \left(q, \frac{r}{2}\right)}{2 \sqrt{2}}|\Sigma|^{\frac{1}{2}+\frac{1}{r}-\frac{1}{q}} & \text { if } r \geq q \text { and } q \geq 2, \\ \frac{q^{*}}{4 \sqrt{2}}|\Sigma|^{\frac{1}{2}+\frac{1}{r}-\frac{1}{q}} & \text { if } 1<r \leq q \text { and } 1<q<2, \\ \frac{\max \left(q, \frac{r}{2}\right)}{2 \sqrt{2}}|\Sigma|^{\frac{1}{2}+\frac{1}{r}-\frac{1}{q}} & \text { if } q<r \leq q^{*} \text { and } 1<q<2 .\end{cases}
$$

Proof. The Sobolev inequality (3.8) is classical and follows by combining the following interpolation (see Lemma II.3.2 in [11])

$$
\|\boldsymbol{u}\|_{r} \leq\left(\frac{\max \left(q, \frac{r}{2}\right)}{2 \sqrt{2}}\right)^{\lambda}\|\boldsymbol{u}\|_{q}^{1-\lambda}\|\nabla \boldsymbol{u}\|_{q}^{\lambda} \quad \text { with } \lambda=\frac{2(r-q)}{r q}
$$

with the inequality $\|\boldsymbol{u}\|_{q} \leq|\Sigma|^{\frac{1}{q}-\frac{1}{r}}\|\boldsymbol{u}\|_{r}$.

Next, we consider a particular version of the Poincaré inequality.

Lemma 3.4 For all $\boldsymbol{u} \in \boldsymbol{W}_{0}^{1, q}(\Sigma), 1 \leq q \leq+\infty$, the following estimate holds

$$
\|\boldsymbol{u}\|_{q} \leq\left\|\frac{\partial \boldsymbol{u}}{\partial x_{1}}\right\|_{q} .
$$

Proof. The result follows from Theorem II.5.1 in [11] by observing that $\Sigma \subset]-1,1[\times \mathbb{R}$.

As well known (see [11]), the standard Poincaré inequality is given by

$$
\|\boldsymbol{u}\|_{q} \leq S_{q, q}\|\nabla \boldsymbol{u}\|_{q}
$$

and consequently, the Poincaré constant in (3.9) is not necessarily optimal. However, since $B$ is independent of the variable $x_{1}$, a direct consequence of (3.9) is that

$$
\|\boldsymbol{u}\|_{q, B} \leq\left\|\frac{\partial \boldsymbol{u}}{\partial x_{1}}\right\|_{q, B} \leq\|\nabla \boldsymbol{u}\|_{q, B} .
$$

This property is particularly useful to establish the Korn inequality in the shear-thinning case.

Finally, we establish some estimates useful in the approximation analysis of the solutions with respect to the parameter $\delta$. 
Lemma 3.5 Let $u, v$ be in $W_{0}^{1, q}(\Sigma)$ with $q \geq 2$, and let $\sigma$ be a continous function such that

$$
|\sigma(\lambda)| \leq c_{0}|\lambda|^{\alpha} \quad \text { with } c_{0}>0 .
$$

Then for every $\alpha \geq 0$, we have

$$
\begin{gathered}
|(\sigma(u), v)| \leq c_{0} D_{q, \alpha}\|\nabla u\|_{q}^{\alpha}\|\nabla v\|_{q}, \\
\|\sigma(u)\|_{q^{\prime}} \leq c_{0} E_{q, \alpha}\|\nabla u\|_{2}^{\alpha},
\end{gathered}
$$

where $D_{q, \alpha}=\max \left(\frac{q}{2 \sqrt{2}}, \frac{\alpha q}{4 \sqrt{2}}\right)^{\alpha+1}|\Sigma|^{\frac{1}{q^{\prime}}+\frac{\alpha+1}{2}-\frac{\alpha}{q}}$ and $E_{q, \alpha}=\max \left(\frac{1}{\sqrt{2}}, \frac{\alpha}{2 \sqrt{2}}\right)^{\alpha}|\Sigma|^{\frac{1}{q^{\prime}}}$.

Proof. Let us first assume that $\alpha \geq 1$. Then, by using the Hölder inequality and the Sobolev inequality (3.8) we obtain

$$
\begin{aligned}
|(\sigma(u), v)| & \leq\|\sigma(u)\|_{q}\|v\|_{q^{\prime}} \leq c_{0}\|u\|_{\alpha q}^{\alpha}\|v\|_{q^{\prime}} \leq c_{0}|\Sigma|^{\frac{1}{q^{\prime}}-\frac{1}{\alpha q}}\|u\|_{\alpha q}^{\alpha}\|v\|_{\alpha q} \\
& \leq c_{0}|\Sigma|^{\frac{1}{q^{\prime}}-\frac{1}{\alpha q}}\left(S_{q, \alpha q}\right)^{\alpha+1}\|\nabla u\|_{q}^{\alpha}\|\nabla v\|_{q}
\end{aligned}
$$

which gives (3.13). Similarly,

$$
\begin{aligned}
\|\sigma(u)\|_{q^{\prime}} & \leq|\Sigma|^{\frac{1}{q^{\prime}}-\frac{1}{2}}\|\sigma(u)\|_{2} \leq c_{0}|\Sigma|^{\frac{1}{q^{\prime}}-\frac{1}{2}}\|u\|_{2 \alpha}^{\alpha} \\
& \leq c_{0}|\Sigma|^{\frac{1}{q^{\prime}}-\frac{1}{2}}\left(S_{2,2 \alpha}\right)^{\alpha}\|\nabla u\|_{2}^{\alpha}
\end{aligned}
$$

and we obtain (3.14). If $\alpha \leq 1$ then

$$
\begin{aligned}
|(\sigma(u), v)| & \leq\|\sigma(u)\|_{\frac{q}{\alpha}}\|v\|_{\left(\frac{q}{\alpha}\right)^{\prime}} \leq c_{0}\|u\|_{q}^{\alpha}\|v\|_{\frac{q}{q-\alpha}} \leq c_{0}|\Sigma|^{\frac{1}{q^{\prime}}-\frac{\alpha}{q}}\|u\|_{q}^{\alpha}\|v\|_{q} \\
& \leq c_{0}|\Sigma|^{\frac{1}{q^{\prime}}-\frac{\alpha}{q}}\left(S_{q, q}\right)^{\alpha+1}\|\nabla u\|_{q}^{\alpha}\|\nabla v\|_{q}
\end{aligned}
$$

and

and the claimed result is proved.

$$
\begin{aligned}
\|\sigma(u)\|_{q^{\prime}} & \leq|\Sigma|^{\frac{1}{q^{\prime}}-\frac{\alpha}{2}}\|\sigma(u)\|_{\frac{2}{\alpha}} \leq c_{0}|\Sigma|^{\frac{1}{q^{\prime}}-\frac{\alpha}{2}}\|u\|_{2}^{\alpha} \\
& \leq c_{0}|\Sigma|^{\frac{1}{q^{\prime}}-\frac{\alpha}{2}}\left(S_{2,2}\right)^{\alpha}\|\nabla u\|_{2}^{\alpha}
\end{aligned}
$$

Lemma 3.6 Let $u, v \in W_{0}^{1, q}(\Sigma)$ with $\frac{3}{2} \leq q<2$, and let $\sigma$ be a continous function satisfying (3.12). Then

$$
\begin{gathered}
|(\sigma(u), v)| \leq c_{0} D_{q, \alpha}\|\nabla u\|_{q}^{\alpha}\|\nabla v\|_{q} \quad \text { for every } 0 \leq \alpha \leq q^{*}-1, \\
\|\sigma(u)\|_{q^{\prime}} \leq c_{0} E_{q, \alpha}\|\nabla u\|_{q}^{\alpha} \quad \text { for every } \frac{1}{q^{\prime}}<\alpha \leq \frac{q^{*}}{q^{\prime}},
\end{gathered}
$$

where $D_{q, \alpha}=\left(S_{q, q^{*}}\right)^{\alpha+1}, E_{q, \alpha}=\left(S_{q, \alpha q^{\prime}}\right)^{\alpha}$ and $q^{*}=\frac{2 q}{2-q}$.

Proof. Notice first that the Sobolev inequality (3.8) is valid for $1<r \leq q^{*}$ if $1 \leq q<2$. If $0<\alpha \leq q^{*}-1$, then $\sigma(u) v$ belongs to $L^{1}(\Sigma)$ and by using (3.8), we obtain

$$
\begin{aligned}
|(\sigma(u), v)| & \leq c_{0}\left\|u^{\alpha}\right\|_{\frac{q^{*}}{\alpha}}\|v\|_{q^{*}} \leq c_{0}\|u\|_{q^{*}}^{\alpha}\|v\|_{q^{*}} \\
& \leq c_{0}\left(S_{q, q^{*}}\right)^{\alpha+1}\|\nabla u\|_{q}^{\alpha}\|\nabla v\|_{q}
\end{aligned}
$$

which gives (3.15). To prove the last estimate, we observe that if $\frac{1}{q^{\prime}}<\alpha \leq \frac{q^{*}}{q^{\prime}}$ then $1<\alpha q^{\prime} \leq q^{*}$. By using (3.8) we obtain

$$
\|\sigma(u)\|_{q^{\prime}} \leq c_{0}\|u\|_{\alpha q^{\prime}}^{\alpha} \leq c_{0}\left(S_{q, \alpha q^{\prime}}\right)^{\alpha}\|\nabla u\|_{q}^{\alpha}
$$

and the claimed result is proved. 


\section{Weak formulation and statement of the main results}

To give a sense to the weak solution of (2.7), let us recall that $B=1+\delta x_{2}$ does not depend on $x_{1}$ and notice that if $\boldsymbol{S}$ is a symmetric tensor, then we have

$$
\begin{aligned}
\left(\nabla^{\star} \cdot \boldsymbol{S}\right)_{1} & =\frac{\partial S_{11}}{\partial x_{1}}+\frac{\partial S_{21}}{\partial x_{2}}+\frac{\delta}{B} S_{21}=\frac{1}{B}\left(\frac{\partial\left(B S_{11}\right)}{\partial x_{1}}+\frac{\partial\left(B S_{21}\right)}{\partial x_{2}}\right) \\
& =\frac{1}{B}(\nabla \cdot(B \boldsymbol{S}))_{1} \\
\left(\nabla^{\star} \cdot \boldsymbol{S}\right)_{2} & =\frac{\partial S_{12}}{\partial x_{1}}+\frac{\partial S_{22}}{\partial x_{2}}+\frac{\delta}{B}\left(S_{22}-S_{33}\right)=\frac{1}{B}\left(\frac{\partial\left(B S_{12}\right)}{\partial x_{1}}+\frac{\partial\left(B S_{22}\right)}{\partial x_{2}}\right)-\frac{\delta}{B} S_{33} \\
& =\frac{1}{B}(\nabla \cdot(B \boldsymbol{S}))_{2}-\frac{\delta}{B} S_{33}, \\
\left(\nabla^{\star} \cdot \boldsymbol{S}\right)_{3} & =\frac{\partial S_{13}}{\partial x_{1}}+\frac{\partial S_{23}}{\partial x_{2}}+\frac{2 \delta}{B} S_{23}=\frac{1}{B}\left(\frac{\partial\left(B S_{13}\right)}{\partial x_{1}}+\frac{\partial\left(B S_{23}\right)}{\partial x_{2}}\right)+\frac{\delta}{B} S_{23} \\
& =\frac{1}{B}(\nabla \cdot(B \boldsymbol{S}))_{3}+\frac{\delta}{B} S_{32},
\end{aligned}
$$

that is

$$
\nabla^{\star} \cdot \boldsymbol{S}=\frac{1}{B} \nabla \cdot(B \boldsymbol{S})+\frac{\delta}{B}\left(S_{23} \mathbf{a}_{3}-S_{33} \mathbf{a}_{2}\right) .
$$

Therefore, if $\boldsymbol{S}$ belongs to in $\boldsymbol{L}^{p^{\prime}}(\Sigma)$ and $\boldsymbol{\varphi}=\left(\varphi_{1}, \varphi_{2}, \varphi_{3}\right) \in \boldsymbol{V}_{B}^{p}$, then an integration by parts shows that

$$
\begin{aligned}
-\left(\nabla^{\star} \cdot \boldsymbol{S}, B \boldsymbol{\varphi}\right) & =-\left(\frac{1}{B} \nabla^{\star} \cdot(B \boldsymbol{S}), B \boldsymbol{\varphi}\right)=-\left(\nabla^{\star} \cdot(B \boldsymbol{S}), \boldsymbol{\varphi}\right) \\
& =-(\nabla \cdot(B \boldsymbol{S}), \boldsymbol{\varphi})-\delta\left(S_{23}, \varphi_{3}\right)+\delta\left(S_{33}, \varphi_{2}\right) \\
& =-\sum_{i=1}^{3}\left(\frac{\partial\left(B S_{1 i}\right)}{\partial x_{1}}+\frac{\partial\left(B S_{2 i}\right)}{\partial x_{2}}, \varphi_{i}\right)-\delta\left(S_{23}, \varphi_{3}\right)+\delta\left(S_{33}, \varphi_{2}\right) \\
& =\sum_{i=1}^{3} \sum_{j=1}^{2}\left(B S_{i j}, \frac{\partial \varphi_{i}}{\partial x_{j}}\right)-\delta\left(S_{23}, \varphi_{3}\right)+\delta\left(S_{33}, \varphi_{2}\right)=\left(\boldsymbol{S}, B D^{\star} \boldsymbol{\varphi}\right) .
\end{aligned}
$$

By taking the inner product of $(2.7)_{1}$ and $B \varphi$ and integrating over $\Sigma$, we obtain the following weak formulation.

Definition 4.1 Assume that $p \geq \frac{3}{2}$. A function $\boldsymbol{u} \in \boldsymbol{V}_{B}^{p}$ is a weak solution of (2.7) if

$$
\left(\boldsymbol{\tau}\left(D^{\star} \boldsymbol{u}\right), B D^{\star} \boldsymbol{\varphi}\right)+\mathcal{R} e\left(B \boldsymbol{u} \cdot \nabla^{\star} \boldsymbol{u}, \boldsymbol{\varphi}\right)=\left(G, \varphi_{3}\right) \quad \text { for all } \boldsymbol{\varphi} \in \boldsymbol{V}_{B}^{p}
$$

As in the case of bounded domains, this definition in meaningful for $p \geq \frac{3}{2}$ and will be used when considering both shear-thinning and shear-thickening flows. The restriction on the exponent $p$ ensures that the convective term belongs to $\boldsymbol{L}^{1}$ when considering test functions in $\boldsymbol{V}_{B}^{p}$.

This formulation can be splitted into two coupled formulations, respectively associated to $u=$ $\left(u_{1}, u_{2}, 0\right)$ and to $\left(0,0, u_{3}\right)$. Indeed, by setting $\varphi=\left(\varphi_{1}, \varphi_{2}, 0\right)$ in (4.1), we can easily see that $u$ satisfies

$$
\left(\tau\left(D^{\star} \boldsymbol{u}\right), B D \varphi\right)+\delta\left(\tau_{33}\left(D^{\star} \boldsymbol{u}\right), \varphi_{2}\right)+\mathcal{R} e(B u \cdot \nabla u, \varphi)=\mathcal{R} e \delta\left(u_{3}^{2}, \phi_{2}\right),
$$

where $\tau=\left(\tau_{i, j}\right)_{i, j=1,2}$. Similarly, by setting $\varphi=\left(0,0, \varphi_{3}\right)$ in (4.1), we see that $u_{3}$ satisfies

$$
\begin{aligned}
& \left(\tau_{13}\left(D^{\star} \boldsymbol{u}\right), B \frac{\partial \varphi_{3}}{\partial x_{1}}\right)+\left(\tau_{23}\left(D^{\star} \boldsymbol{u}\right), B \frac{\partial \varphi_{3}}{\partial x_{2}}-\delta \varphi_{3}\right)+\mathcal{R} e\left(\left(B u \cdot \nabla u_{3}, \varphi_{3}\right)+\delta\left(u_{3} u_{2}, \varphi_{3}\right)\right) \\
& =\left(G, \varphi_{3}\right) .
\end{aligned}
$$

In the case of the Navier-Stokes equations, both formulations (4.1) and (4.2)-(4.3) can be used to establish the existence and uniqueness of a weak solution. In the present paper, the corresponding 
existence and uniqueness results are obtained as a particular case (by setting $p=2$ ) using the first formulation, while similar results were established in [10, by using the second formulation. One notable difference however is related with the corresponding estimates: involving $\boldsymbol{u}$ and $w_{3}$ and independent of the Reynolds number in the first case, involving $u$ and $w_{3}$ (and thus $\boldsymbol{u}$ ) and depending on $\delta \mathcal{R} e$ (at least for $u$ and $\boldsymbol{u}$ ) in the second one. This fact has no influence on the study of the solvability of our problem, but a different estimate on $u$ is missed if we do not consider (4.2). When dealing with the generalized Navier-Stokes equations (2.7), the global formulation proves much more suitable than the splitting one. Because of the nonlinearity of the shear-stress, the coercivity property in the case $p<2$ and the monotonicity property in both cases $p<2$ and $p>2$ fail to be satisfied separately for $u$ and $u_{3}$. We will use the formulation (4.2) a posteriori to derive an additional estimate for $u$ that highlights the dependance on the curvature ratio and the Reynolds number.

Existence and uniqueness of a weak solution in the shear-thickening case, together with associated estimates, is considered in the next result.

Theorem 4.2 Assume that $p \geq 2$. Then problem (2.7) admits at least a weak solution $\boldsymbol{u} \in \boldsymbol{V}_{B}^{p}$, and this solution satisfies

$$
\begin{aligned}
& \left\|D^{\star} \boldsymbol{u}\right\|_{2, B} \leq \kappa_{1}, \\
& \left\|D^{\star} \boldsymbol{u}\right\|_{p, B} \leq \kappa_{1}^{\frac{2}{p}}, \\
& \left\|\nabla u_{3}\right\|_{2, B} \leq 2^{\frac{1}{2}} \kappa_{1}, \\
& \|D u\|_{2, B} \leq \kappa_{2} \delta \mathcal{R} e, \\
& \|D u\|_{p, B} \leq\left(\kappa_{2} \delta \mathcal{R} e\right)^{\frac{2}{p}}
\end{aligned}
$$

with

$$
\kappa_{1}=\left(\frac{m}{2}\right)^{\frac{1}{2}}|G||\Sigma| \quad \text { and } \quad \kappa_{2}=\frac{m^{\frac{3}{2}}|\Sigma|}{2} \kappa_{1}^{2} .
$$

Moreover, if the Reynolds number $\mathcal{R} e$ is such that

$$
\mathcal{R} e<\frac{2}{\kappa_{1} \kappa_{3}} \quad \text { with } \kappa_{3}=n m^{\frac{3}{2}}|\Sigma|^{\frac{3}{4}}
$$

then problem (2.7) admits a unique weak solution.

Unlike the estimates given in (4.7)-(4.8), the estimates in (4.4)-(4.5), and consequently in (4.6), are uniformly bounded and neither depend on $\delta$ nor on $\mathcal{R} e$. On the other hand, the estimates in (4.7)-(4.8) show that the secondary shear-thickening flows, if they exist, depend simultaneously on the pipe curvature ratio and on the Reynolds number or equivalently, and after introducing the standard adimensionalization $\left(\tilde{u}, \tilde{u}_{3}\right)=\left(\sqrt{\delta} u, u_{3}\right)$, that they depend on the Dean number $\mathcal{D} e=\sqrt{\delta} \mathcal{R} e$. These results are specially useful when dealing with small Dean numbers and imply, in this situation, that the shear-thickening secondary flows are necessarily small.

Similar results are obtained in the shear-thinning case.

Theorem 4.3 Assume that $\frac{3}{2} \leq p<2$. Then problem (2.7) admits at least a weak solution $\boldsymbol{u} \in \boldsymbol{V}_{B}^{p}$, and this solution satisfies

$$
\begin{aligned}
& \left\|D^{\star} \boldsymbol{u}\right\|_{p, B} \leq \kappa_{2}, \\
& \left\|D^{\star} \boldsymbol{u}\right\|_{p, B}^{p} \leq \kappa_{3}, \\
& \left\|\nabla u_{3}\right\|_{p, B} \leq \kappa_{4}, \\
& \|D u\|_{p, B} \leq \kappa_{5} \delta \mathcal{R} e
\end{aligned}
$$


with

$$
\begin{aligned}
& \kappa_{1}=\frac{m^{\frac{1}{p}}}{2}|G|\left\|\left.\Sigma\right|^{\frac{1}{p^{\prime}}}, \quad \kappa_{2}=\kappa_{1}\left(\frac{\|B\|_{1}}{p-1}+\kappa_{1}^{p^{\prime}}\right)^{\frac{2-p}{p}}, \quad \kappa_{3}=p^{\prime}\right\| B \|_{1}+\left(2^{\frac{2-p}{2}} \kappa_{1}\right)^{p^{\prime}}, \\
& \kappa_{4}=2^{2-p}(1+\delta m) \kappa_{2},
\end{aligned}
$$

and where $\kappa_{5}$ is a positive constant only depending on $\Sigma, p, G$ and $m$. Moreover, there exists a positive constant $\kappa_{6}$ only depending on $\Sigma, p, G, m$ and $n$ such that if

$$
\mathcal{R} e<\frac{1}{2 \kappa_{1} \kappa_{6}}\left(\frac{\|B\|_{1}}{p-1}+\kappa_{1}^{p^{\prime}}\right)^{-\frac{2(2-p)}{p}},
$$

then problem (2.7) admits a unique weak solution.

As in the case of shear-thickening flows, the estimates in (4.10)-(4.12) are uniformly bounded and independent of $\delta$ and $\mathcal{R} e$ while estimate (4.13) depends simultaneously on these two parameters and shows that if the Dean number is small, then the shear-thinning secondary flows are necessarily small.

Remark 4.4 In the statement of Theorem 4.3, the dependence of the constants $\kappa_{5}$ and $\kappa_{6}$ on the parameters $\delta$ and $m$ is explicitely known. More precisely, we have

$$
\begin{aligned}
& \kappa_{5}=\frac{m^{\frac{3}{p}}}{C_{K, 1}}\left(S_{p, 2 p^{\prime}}\right)^{3} \kappa_{4}^{2}\left(\frac{\|B\|_{1}}{p-1}+\kappa_{1}^{p^{\prime}}\right)^{\frac{2-p}{p}}, \\
& \kappa_{6}=\frac{\frac{8 n^{\frac{p+1}{p}} m^{\frac{6}{p}}(1+\delta m)^{3}}{C_{K, 1}^{3}}\left(S_{p, 2 p^{\prime}}\right)^{2}}{},
\end{aligned}
$$

where $C_{K, 1}$ is the classical Korn inequality in $\boldsymbol{W}_{0}^{1, p}(\Sigma)$. Notice also that the constants $\kappa_{2}, \kappa_{3}$, $\kappa_{4}, \kappa_{5}$ and $\kappa_{6}$ depend on $\kappa_{1}$.

Having a weak solution, the corresponding term $\nabla \pi$ can be constructed by the same way as in the linear case. The pressure is determined up to a constant and becomes unique under the additional condition $\int_{\Sigma} \pi d x=0$.

Corollary 4.5 Assume that $p \geq 2$ and that $\boldsymbol{u} \in \boldsymbol{V}_{B}^{p}$ is a weak solution of (2.7). Then there exists a unique $\pi \in L_{0}^{p^{\prime}}(\Sigma)$ such that (2.7) 1 holds in $\boldsymbol{W}^{-1, p^{\prime}}(\Sigma)$. Moreover, we have the following estimate

$$
\|\pi\|_{p^{\prime}} \leq \kappa\left(\|D u\|_{p, B}+\delta^{2}\left\|u_{2}\right\|_{p, B}+\mathcal{R} e\left(\|D u\|_{p, B}^{2}+\delta\left\|\nabla u_{3}\right\|_{p, B}^{2}\right)\right)
$$

where $\kappa$ is a positive constant only depending on $\Sigma, p, m$ and $n$.

Similarly, existence of the pressure in the shear-thinning case is considered below.

Corollary 4.6 Assume that $\frac{3}{2} \leq p<2$ and that $\boldsymbol{u} \in \boldsymbol{V}_{B}^{p}$ is a weak solution of (2.7). Then there exists a unique $\pi \in L_{0}^{p^{\prime}}(\Sigma)$ such that (2.7) ${ }_{1}$ holds in $\boldsymbol{W}^{-1, p^{\prime}}(\Sigma)$. Moreover, we have the following estimate

$$
\|\pi\|_{p^{\prime}} \leq \kappa\left(\|D u\|_{p, B}^{p-1}+\delta^{p}\left\|u_{2}\right\|_{p, B}^{p-1}+\mathcal{R} e\left(\|D u\|_{p, B}^{2}+\delta\left\|\nabla u_{3}\right\|_{p, B}^{2}\right)\right),
$$

where $\kappa$ is a positive constant only depending on $\Sigma, p, m$ and $n$.

The next results deal with special properties of the solutions of (2.7). We recall that a solution $\left(\boldsymbol{u}=\left(u, u_{3}\right), \pi\right)$ is unidirectional flow if $u_{1}=u_{2}=0$. 
Proposition 4.7 Assume that $p \geq \frac{3}{2}$. If $\delta \mathcal{R} e=0$, then all the solutions of (2.7) are unidirectional flows.

Proof. Let us first assume that $p \geq 2$. Taking into account (4.8), we deduce that $\|\nabla u\|_{p}=0$ and since $u_{\mid \partial \Sigma}=0$, by using the Poincaré inequality it follows that $u=0$ and thus $\boldsymbol{u}=\left(0,0, u_{3}\right)$ with $u_{3}$ satisfying

$$
\begin{cases}-\nabla^{\star}\left(\left(1+\frac{1}{2}\left|\nabla^{\star} u_{3}\right|^{2}\right)^{\frac{p-2}{2}} \nabla^{\star} u_{3}\right)=\frac{G}{B} & \text { in } \Sigma \\ u_{3}=0 & \text { in } \partial \Sigma .\end{cases}
$$

The result corresponding to the shear-thinning case can be proved similarly using the estimate (4.13) instead of the estimate (4.8).

Proposition 4.8 Assume that $p \geq \frac{3}{2}$. If $\delta \mathcal{R} e>0$, then the solutions of (2.7) are not unidirectional flows.

Proof. Let us assume that $\left(u_{1}, u_{2}\right)=(0,0)$. System (2.7) reduces to

$$
\nabla \pi=\mathcal{R} e \frac{\delta}{B} u_{3} \boldsymbol{a}_{2} \quad \text { and } \quad u_{3} \text { satisfies (4.15). }
$$

If $\delta \mathcal{R} e>0$, the first equation implies that $\pi$ (and thus $u_{3}$ ) does not depend on the variable $x_{1}$. Following the arguments developed in [10, we can prove that

$$
u_{3}=0 \quad \text { in } \Sigma \text {. }
$$

Indeed, let $\left(x_{1}, x_{2}\right)$ be an arbitrary point in $\Sigma$ and denote by $\left(x_{0}, x_{2}\right)$ a point on $\partial \Sigma$ that is the intersection of the straight line with origin $\left(x_{1}, x_{2}\right)$, parallel to $\boldsymbol{a}_{1}$ and such that the segment $\operatorname{seg}=\cup_{\alpha \in[0,1]}$ lies in $\bar{\Sigma}$. Since $u_{3}$ is independent of $x_{1}$, it follows that $u\left(x_{1}^{\alpha}, x_{2}\right)$ is independent of $\alpha$ for $x_{1}^{\alpha} \in$ seg. Therefore, by taking into account the boundary condition $u_{\mid \Sigma}=0$, we obtain

$$
u_{3}\left(x_{1}, x_{2}\right)=u_{3}\left(x_{1}^{1}, x_{2}\right)=u_{3}\left(x_{1}^{0}, x_{2}\right)=u_{3}\left(x_{0}, x_{2}\right)=0 .
$$

The point $\left(x_{1}, x_{2}\right)$ being arbitrary in $\Sigma$, we deduce that (4.16) holds, and this contradicts (4.15) and completes the proof.

Let us now analyze the behavior of the weak solutions of (2.7) with respect to the parameter $\delta$. The objective would be to use these results when $\delta$ is small to approximate a solution $\boldsymbol{u}$ of (2.7) by a solution of a similar but simpler system. More precisely, we consider the following problem

$$
\left(E_{\sigma}\right) \quad \begin{cases}-\nabla \cdot(\boldsymbol{\tau}(D \boldsymbol{w}))+\mathcal{R} e \boldsymbol{w} \cdot \nabla \boldsymbol{w}+\nabla \pi=\frac{G}{B} \boldsymbol{a}_{3}+\delta \sigma\left(w_{3}\right) \boldsymbol{a}_{2} & \text { in } \Sigma, \\ \nabla \cdot \boldsymbol{w}=0 & \text { in } \Sigma, \\ \boldsymbol{w}=0 & \text { on } \partial \Sigma,\end{cases}
$$

where $\sigma$ is a non constant function and we aim to estimate the difference $\boldsymbol{u}-\boldsymbol{w}$. Obviously, the considerations concerning the monotonicity and coercivity properties of the global and the coupled formulations described above and the difficulties encountered in the treatment of the full governing equations arise in a similar way for $\left(E_{\sigma}\right)$. Moreover, in the derivation of the a priori estimate, the term involving $\sigma$ induces an additional difficulty that can be overcome by carrying out a careful analysis.

Begining with the shear-thickening case, we summarize the properties of the solutions of $\left(E_{\sigma}\right)$. 
Proposition 4.9 Assume that $p \geq 2$ and let $\sigma$ be a non constant continous function satisfying (3.12) for some $\alpha \geq 0$. Then problem $\left(E_{\sigma}\right)$ admits at least a weak solution $\boldsymbol{w} \in \boldsymbol{V}^{p}$, and this solution satisfies

$$
\begin{array}{ll}
\left\|\nabla w_{3}\right\|_{2} \leq c_{1}, & \left\|\nabla w_{3}\right\|_{p}^{p} \leq 2^{\frac{p-2}{2}} c_{1}^{2}, \\
\|D w\|_{2} \leq c_{0} c_{2} \delta, & \|D w\|_{p}^{p} \leq\left(c_{0} c_{2} \delta\right)^{2},
\end{array}
$$

where

$$
c_{1}=\frac{m|\Sigma||G|}{\sqrt{2}} \quad \text { and } \quad c_{2}=\frac{D_{2, \alpha} c_{1}^{\alpha}}{\sqrt{2}} \text { with } D_{2, \alpha} \text { given in Lemma } 3.5 \text {. }
$$

Moreover, if $\delta>0$ then the solutions of $\left(E_{\sigma}\right)$ are not unidirectional flows.

Next we state the corresponding approximation result.

Proposition 4.10 Assume that the assumptions of Proposition 4.9 are fulfilled and let $\boldsymbol{u}, \boldsymbol{w}$ be the solutions of (2.7) and $\left(E_{\sigma}\right)$. There exists $\mathcal{R} e_{0}>0$ such that if $\mathcal{R} e \leq \mathcal{R} e_{0}$ then

$$
\|D(\boldsymbol{u}-\boldsymbol{w})\|_{p}^{p}+\|D(\boldsymbol{u}-\boldsymbol{w})\|_{2}^{2} \leq \kappa \delta^{p^{\prime}}
$$

where $\kappa$ depends only on $p, \Sigma, m, n, c_{0}$ and $\alpha$.

Similarly, we consider the solvability of $\left(E_{\sigma}\right)$ in the shear-thinning case and the corresponding approximation result.

Proposition 4.11 Assume that $\frac{3}{2} \leq p<2$ and let $\sigma$ is a non constant continuous function satisfying (3.12) for some $\alpha$ such that $\frac{1}{p^{\prime}}<\alpha<\frac{p^{*}}{2 p^{\prime}}$. Then problem $\left(E_{\sigma}\right)$ admits at least a weak solution $\boldsymbol{w} \in \boldsymbol{V}^{p}$, and this solution satisfies

$$
\begin{aligned}
& \left\|\nabla w_{3}\right\|_{p} \leq c_{1}\left(|\Sigma|+c_{4}^{p}\right)^{\frac{2-p}{p}}, \\
& \|D w\|_{p} \leq c_{0} c_{1}^{\alpha} c_{2}\left(|\Sigma|+c_{4}^{p}\right)^{\frac{(2-p)(\alpha+1)}{p}} \delta
\end{aligned}
$$

where

$$
\begin{aligned}
& c_{1}=m|\Sigma|^{\frac{1}{p^{\prime}}}|G|, \quad c_{2}=\frac{D_{p, \alpha}}{2 C_{K, 1}} \quad \text { with } D_{p, \alpha} \text { given in Lemma 3.6. } \\
& c_{3}=\left(c_{1}+c_{0} c_{1}^{\alpha} c_{2}\right)(1+|\Sigma|)^{\frac{(2-p)(\alpha+1)}{p}}, \\
& c_{4}=c_{3}\left(\frac{1}{1-(2-p)(\alpha+1)}+c_{3}^{\frac{1}{1-(2-p)(\alpha+1)}}\right)^{\frac{(2-p)(\alpha+1)}{p}} .
\end{aligned}
$$

Moreover, if $\delta>0$ then the solutions of $\left(E_{\sigma}\right)$ are not unidirectional flows.

Proposition 4.12 Assume that the assumptions of Proposition 4.11 are fulfilled and let $\boldsymbol{u}, \boldsymbol{w}$ be the solutions of (2.7) and $\left(E_{\sigma}\right)$. There exists $\mathcal{R} e_{0}>0$ such that if $\mathcal{R} e \leq \mathcal{R} e_{0}$ then

$$
\|D(\boldsymbol{u}-\boldsymbol{w})\|_{p} \leq \kappa \delta^{p-1}
$$

where $\kappa$ depends only on $p, \Sigma, m, n, c_{0}$ and $\alpha$.

Propositions 4.9, 4.10, 4.11 and 4.12 show that for $\delta>0$ sufficiently small, a solution of (2.7) can be approximated by a solution of $\left(E_{\sigma}\right)$, whose secondary flows exist even if they are proportionately weak. It is worth observing that this result is valid for a relatively large class of functions $\sigma$ and raises an interesting question related with the possible choices for $c_{0}$ and $\alpha$ that would guarantee an optimal approximation, in a sense to be correctly and adequately defined. 
We finish this section by considering the case of Navier-Stokes equations obtained by setting $p=2$. Notice that the constants $\kappa_{1}, \kappa_{2}$ and $\kappa_{3}$ in the statement of Theorem 4.2 are independent of the exponent $p$ and that the condition that guarantees the uniqueness of weak solutions only depends on $\Sigma, G, m$ and $n$. As a consequence, the estimates and the sufficient condition on $\mathcal{R} e$ are identical in the particular case of Newtonian fluids.

Theorem 4.13 The Navier-Stokes problem

$$
\begin{cases}-\nabla^{\star} \cdot\left(2 D^{\star} \boldsymbol{u}\right)+\mathcal{R} e \boldsymbol{u} \cdot \nabla^{\star} \boldsymbol{u}+\nabla \pi=\frac{G}{B} \boldsymbol{a}_{3} & \text { in } \Sigma, \\ \nabla^{\star} \cdot \boldsymbol{u}=0 & \text { in } \Sigma, \\ \boldsymbol{u}=0 & \text { on } \partial \Sigma,\end{cases}
$$

admits at least a weak solution $\boldsymbol{u} \in \boldsymbol{V}_{B}^{2}$. This solution satisfies the estimates (4.4), (4.6), (4.7) and if $\mathcal{R} e$ satisfies (4.9), then the solution is unique. If $\delta \mathcal{R} e=0$, then all the solutions are unidirectional flows, otherwise they are not unidirectional flows. Finally, let $\sigma$ be a non constant continuous function satisfying (3.12) for some $\alpha \geq 0$. Then the following problem

$$
\begin{cases}-\Delta \boldsymbol{w}+\mathcal{R} e \boldsymbol{w} \cdot \nabla \boldsymbol{w}+\nabla \pi=\frac{G}{B} \boldsymbol{a}_{3}+\delta \sigma\left(w_{3}\right) \boldsymbol{a}_{2} & \text { in } \Sigma, \\ \nabla \cdot \boldsymbol{w}=0 & \text { in } \Sigma, \\ \boldsymbol{w}=0 & \text { on } \partial \Sigma,\end{cases}
$$

admits a weak solution in $\boldsymbol{V}^{2}$. Moreover, there exists $\mathcal{R} e_{0}>0$ such that if $\mathcal{R} e \leq \mathcal{R} e_{0}$ then

$$
\|D(\boldsymbol{u}-\boldsymbol{w})\|_{2} \leq \kappa \delta
$$

where $\kappa$ depends on $\Sigma, m, n, c_{0}$ and $\alpha$.

The case of Navier-Stokes equations has been fully studied in [10]. In the previous result, we recover similar results with some differences concerning the analysis with respect to $\delta$. On the one hand, our estimate is valid for a class of problems larger than the classical Dean problem obtained by setting

$$
\sigma(\lambda)=\mathcal{R} e \lambda^{2}
$$

On the other hand, the estimate corresponding to the secondary flows is less accurate. Indeed, after introducing the adimensionalization $\left(\tilde{u}, \tilde{u}_{3}\right)=\left(\sqrt{\delta} u, u_{3}\right)$, we obtain

$$
\|D(\tilde{u}-\tilde{w})\|_{2} \leq \kappa \sqrt{\delta}, \quad\left\|\nabla\left(\tilde{u}_{3}-\tilde{w}_{3}\right)\right\|_{2} \leq \kappa \delta
$$

while the estimate obtained in [10] reads as

$$
\|D(\tilde{u}-\tilde{w})\|_{2} \leq \kappa \delta, \quad\left\|\nabla\left(\tilde{u}_{3}-\tilde{w}_{3}\right)\right\|_{2} \leq \kappa \delta .
$$

This is due to some technical difficulties mainly related with the combined effect of $D u$ and $\nabla u_{3}$ in the shear-rate and its consequences on the monotonicity properties of the tensor $\boldsymbol{\tau}$. Indeed, in the case of Navier-Stokes equations with $\sigma$ given by (4.17), the corresponding coupled formulations allow to derive, in a first step, estimates for $\|D(\tilde{u}-\tilde{w})\|_{2}$ and $\left\|\nabla\left(\tilde{u}_{3}-\tilde{w}_{3}\right)\right\|_{2}$ dependent on one another. The combination of these estimates in a second step gives the result. In the case of a shear-dependent viscosity, and as already observed concerning the solvability of problem (2.7), the lack of monotonicity of the tensores $\tau=\left(\tau_{i j}\right)_{i, j=1,2}, \tau_{13}$ and $\tau_{23}$ prevents from using the same arguments, and the global estimates we obtain come with a cost. 


\section{$5 \quad$ Shear-thickening flows}

The aim of this section is to study the case of shear-thickening flows (corresponding to $p \geq 2$ ). To achieve this goal, we first establish a Korn inequality, and then estimate the convective term as well as the extra stress tensor in an adequate setting. We finally prove the corresponding main results given above.

\subsection{On the Korn inequality}

The next result deals with an inequality of Korn's type in $\boldsymbol{H}_{0}^{1}(\Sigma)$, very similar to the classical one but involving the operators $\nabla^{\star}$ and $D^{\star}$.

Lemma 5.1 Let $\boldsymbol{u}=\left(u_{1}, u_{2}, u_{3}\right) \in \boldsymbol{H}_{0}^{1}(\Sigma)$. Then

$$
\left\|\nabla^{\star} \boldsymbol{u}\right\|_{2, B}^{2}=2\left\|D^{\star} \boldsymbol{u}\right\|_{2, B}^{2}-\left\|\frac{1}{B} \nabla \cdot(B u)\right\|_{2, B}^{2} .
$$

Proof. The definition of $D^{\star}$ together with standard calculations show that

$$
\begin{aligned}
2\left\|D^{\star} \boldsymbol{u}\right\|_{2, B}^{2} & =2\|D u\|_{2, B}^{2}+2\left\|\frac{\delta}{\sqrt{B}} u_{2}\right\|_{2}^{2}+\left\|\frac{\partial u_{3}}{\partial x_{1}}\right\|_{2, B}^{2}+\left\|\sqrt{B} \frac{\partial u_{3}}{\partial x_{2}}-\frac{\delta}{\sqrt{B}} u_{3}\right\|_{2}^{2} \\
& =2\|D u\|_{2, B}^{2}+2\left\|\frac{\delta}{\sqrt{B}} u_{2}\right\|_{2}^{2}+\left\|\nabla u_{3}\right\|_{2, B}^{2}+\left\|\frac{\delta}{\sqrt{B}} u_{3}\right\|_{2}^{2} \\
& =2\|D u\|_{2, B}^{2}+2\left\|\frac{\delta}{B} u_{2}\right\|_{2, B}^{2}+\left\|\nabla u_{3}\right\|_{2, B}^{2}+\left\|\frac{\delta}{B} u_{3}\right\|_{2, B}^{2},
\end{aligned}
$$

where $u=\left(u_{1}, u_{2}, 0\right)$. Since

$$
\nabla \cdot(2 D u)-\Delta u=\nabla \cdot\left((\nabla u)^{T}\right)=\nabla(\nabla \cdot u),
$$

we deduce that for every $\varphi \in \boldsymbol{H}_{0}^{1}(\Sigma)$ we have

$$
(\nabla u, \nabla \varphi)=2(D u, \nabla \varphi)-(\nabla \cdot u, \nabla \cdot \varphi) .
$$

On the other hand, easy calculations show that $D u: \nabla \varphi=D u: D \varphi$. Combining these identities, we obtain

$$
(\nabla u, \nabla \varphi)=2(D u, D \varphi)-(\nabla \cdot u, \nabla \cdot \varphi)
$$

and thus

$$
\begin{aligned}
(\nabla u, \nabla(B \boldsymbol{\varphi})) & =(B \nabla u, \nabla \boldsymbol{\varphi})+\delta\left(\frac{\partial u}{\partial x_{2}}, \boldsymbol{\varphi}\right) \\
& =2(D u, D(B \boldsymbol{\varphi}))-(\nabla \cdot u, \nabla \cdot(B \boldsymbol{\varphi})) \\
& =2(B D u, D \boldsymbol{\varphi})+\delta\left(\frac{\partial u}{\partial x_{2}}+\nabla u_{2}, \boldsymbol{\varphi}\right)-(\nabla \cdot u, \nabla \cdot(B \boldsymbol{\varphi})) \\
& =2(B D u, D \boldsymbol{\varphi})+\delta\left(\frac{\partial u}{\partial x_{2}}, \boldsymbol{\varphi}\right)-\delta\left(u_{2}, \nabla \cdot \boldsymbol{\varphi}\right)-(\nabla \cdot u, \nabla \cdot(B \boldsymbol{\varphi})) \\
& =2(B D u, D \boldsymbol{\varphi})+\delta\left(\frac{\partial u}{\partial x_{2}}, \boldsymbol{\varphi}\right)+\left(\frac{\delta^{2}}{B} u_{2}, \varphi_{2}\right)-\left(\frac{1}{B} \nabla \cdot(B u), \nabla \cdot(B \boldsymbol{\varphi})\right) .
\end{aligned}
$$

The last equality implies that

$$
(B \nabla u, \nabla \boldsymbol{\varphi})=2(B D u, D \varphi)-\left(\frac{1}{B} \nabla \cdot(B u), \nabla \cdot(B \boldsymbol{\varphi})\right)+\left(\frac{\delta^{2}}{B} u_{2}, v_{2}\right)
$$


for all $\varphi \in \boldsymbol{H}_{0}^{1}(\Sigma)$. Setting $\varphi=u$, we obtain

$$
\begin{aligned}
\|\nabla u\|_{2, B}^{2} & =2\|D u\|_{2, B}^{2}+\left\|\frac{\delta}{\sqrt{B}} u_{2}\right\|_{2}^{2}-\left\|\frac{1}{\sqrt{B}} \nabla \cdot(B u)\right\|_{2}^{2} \\
& =2\|D u\|_{2, B}^{2}+\left\|\frac{\delta}{B} u_{2}\right\|_{2, B}^{2}-\left\|\frac{1}{B} \nabla \cdot(B u)\right\|_{2, B}^{2} .
\end{aligned}
$$

On the other hand, by taking into acount the definition of $\nabla^{\star}$ we have

$$
\left\|\nabla^{\star} \boldsymbol{u}\right\|_{2, B}^{2}=\|\nabla \boldsymbol{u}\|_{2, B}^{2}+\left\|\frac{\delta}{B} u_{2}\right\|_{2, B}^{2}+\left\|\frac{\delta}{B} u_{3}\right\|_{2, B}^{2} .
$$

The conclusion follows from (5.2), (5.3) and (5.4).

Remark 5.2 A direct consequence of Lemma 5.1 is that

$$
\left\|\nabla^{\star} \boldsymbol{u}\right\|_{2, B}^{2} \leq 2\left\|D^{\star} \boldsymbol{u}\right\|_{2, B}^{2} \quad \text { for all } \boldsymbol{u} \in \boldsymbol{H}_{0}^{1}(\Sigma)
$$

and the equality holds if $\nabla \cdot(B \boldsymbol{u})=0$.

\subsection{Estimates on the convective term}

We point out some notable facts related with the trilinear forms $a$ and $a_{\star}$ defined by

$$
\begin{aligned}
& a(\boldsymbol{u}, \boldsymbol{v}, \boldsymbol{w})=(\boldsymbol{u} \cdot \nabla \boldsymbol{v}, \boldsymbol{w}), \\
& a_{\star}(\boldsymbol{u}, \boldsymbol{v}, \boldsymbol{w})=\left(\boldsymbol{u} \cdot \nabla^{\star} \boldsymbol{v}, \boldsymbol{w}\right)=a(\boldsymbol{u}, \boldsymbol{v}, \boldsymbol{w})+\left(\frac{\delta}{B} v_{3} u_{2}, w_{3}\right)-\left(\frac{\delta}{B} v_{3} u_{3}, w_{2}\right) .
\end{aligned}
$$

Lemma 5.3 Assume that $p \geq \frac{3}{2}$. For every $\boldsymbol{u} \in \boldsymbol{V}_{B}^{p}$ and every $\boldsymbol{v}, \boldsymbol{w} \in \boldsymbol{W}_{0}^{1, p}(\Sigma)$, we have

$$
a_{\star}(B \boldsymbol{u}, \boldsymbol{v}, \boldsymbol{v})=0 \quad \text { and } \quad a_{\star}(B \boldsymbol{u}, \boldsymbol{v}, \boldsymbol{w})=-a_{\star}(B \boldsymbol{u}, \boldsymbol{w}, \boldsymbol{v}) .
$$

Proof. Taking into account the definition of $a_{\star}$ and the fact that $\nabla \cdot(B \boldsymbol{u})=0$, we deduce that

$$
a_{\star}(B \boldsymbol{u}, \boldsymbol{v}, \boldsymbol{v})=a(B \boldsymbol{u}, \boldsymbol{v}, \boldsymbol{v})+\delta\left(u_{3} v_{2}, v_{3}\right)-\delta\left(u_{3}, v_{3} v_{2}\right)=a(B \boldsymbol{u}, \boldsymbol{v}, \boldsymbol{v})=0 .
$$

Similarly,

$$
\begin{aligned}
a_{\star}(B \boldsymbol{u}, \boldsymbol{v}, \boldsymbol{w}) & =a(B \boldsymbol{u}, \boldsymbol{v}, \boldsymbol{w})+\delta\left(u_{3} v_{2}, w_{3}\right)-\delta\left(u_{3} v_{3}, w_{2}\right) \\
& =-a(B \boldsymbol{u}, \boldsymbol{w}, \boldsymbol{v})+\delta\left(u_{3} v_{2}, w_{3}\right)-\delta\left(u_{3} v_{3}, w_{2}\right)=-a_{\star}(B \boldsymbol{u}, \boldsymbol{w}, \boldsymbol{v})
\end{aligned}
$$

and the proof is complete.

Lemma 5.4 Let $\boldsymbol{u}, \boldsymbol{v}$ and $\boldsymbol{w}$ be in $\boldsymbol{H}_{0}^{1}(\Sigma)$. Then the following estimate holds

$$
\left|a_{\star}(\boldsymbol{u}, \boldsymbol{v}, B \boldsymbol{w})\right| \leq \kappa_{3}\left\|D^{\star} \boldsymbol{u}\right\|_{2, B}\left\|D^{\star} \boldsymbol{v}\right\|_{2, B}\left\|D^{\star} \boldsymbol{w}\right\|_{2, B},
$$

where $\kappa_{3}=n m^{\frac{3}{2}}|\Sigma|^{\frac{3}{4}}$.

Proof. Setting $r=4$ and $q=2$ in the Sobolev inequality (3.8), we obtain

$$
\begin{aligned}
\left|a_{\star}(\boldsymbol{u}, \boldsymbol{v}, B \boldsymbol{w})\right| & \leq n\|\boldsymbol{u}\|_{4}\left\|\nabla^{\star} \boldsymbol{v}\right\|_{2}\|\boldsymbol{w}\|_{4} \leq n\left(S_{2,4}\right)^{2}\|\nabla \boldsymbol{u}\|_{2}\left\|\nabla^{\star} \boldsymbol{v}\right\|_{2}\|\nabla \boldsymbol{w}\|_{2} \\
& \leq n m^{\frac{3}{2}}\left(S_{2,4}\right)^{2}\left\|\nabla^{\star} \boldsymbol{u}\right\|_{2, B}\left\|\nabla^{\star} \boldsymbol{v}\right\|_{2, B}\left\|\nabla^{\star} \boldsymbol{w}\right\|_{2, B} .
\end{aligned}
$$

The estimate follows then by taking into account Remark 5.2 . 


\subsection{Estimates on the extra stress tensor}

Our aim now is to establish some continuity, coercivity and monotonicity results for the stress tensor $\tau$.

Proposition 5.5 Assume that $p \geq 2$ and let $\boldsymbol{f}, \boldsymbol{g} \in \boldsymbol{L}^{p}\left(\Sigma, \mathbb{R}^{3 \times 3}\right)$. Then the following estimates hold

Continuity.

$$
\begin{gathered}
\left\|\left(1+|\boldsymbol{f}|^{2}\right)^{\frac{p-2}{2}} \boldsymbol{g}\right\|_{p^{\prime}, B} \leq \mathcal{F}_{B}\left(\|\boldsymbol{f}\|_{p, B}\right)\|\boldsymbol{g}\|_{p, B} \\
\|\boldsymbol{\tau}(\boldsymbol{f})-\boldsymbol{\tau}(\boldsymbol{g})\|_{p^{\prime}, B} \leq(p-1) \mathcal{F}_{B}\left(\|\boldsymbol{f}\|_{p, B}+\|\boldsymbol{g}\|_{p, B}\right)\|\boldsymbol{f}-\boldsymbol{g}\|_{p, B}
\end{gathered}
$$

Coercivity.

$$
(\boldsymbol{\tau}(\boldsymbol{f}), B \boldsymbol{f}) \geq 2\|\boldsymbol{f}\|_{2, B}^{2}, \quad(\boldsymbol{\tau}(\boldsymbol{f}), B \boldsymbol{f}) \geq 2\|\boldsymbol{f}\|_{p, B}^{p}
$$

Monotonicity.

$$
\begin{aligned}
& (\boldsymbol{\tau}(\boldsymbol{f})-\boldsymbol{\tau}(\boldsymbol{g}), B(\boldsymbol{f}-\boldsymbol{g})) \geq 2\|\boldsymbol{f}-\boldsymbol{g}\|_{2, B}^{2}, \\
& (\boldsymbol{\tau}(\boldsymbol{f})-\boldsymbol{\tau}(\boldsymbol{g}), B(\boldsymbol{f}-\boldsymbol{g})) \geq \frac{1}{2^{p-1}(p-1)}\|\boldsymbol{f}-\boldsymbol{g}\|_{p, B}^{p} .
\end{aligned}
$$

where $\mathcal{F}_{B}(\lambda)=\left(\left\|B^{\frac{1}{p}}\right\|_{p}+\lambda\right)^{p-2}$.

Proof. Assume that $p>2$. Standard calculation show that

$$
\left\|\left(1+|\boldsymbol{f}|^{2}\right)^{\frac{p-2}{2}} \boldsymbol{g}\right\|_{p^{\prime}, B} \leq\left\|\left(1+|\boldsymbol{f}|^{2}\right)^{\frac{p-2}{2}}\right\|_{\frac{p}{p-2}, B}\|\boldsymbol{g}\|_{p, B} \leq\left(\left\|B^{\frac{1}{p}}\right\|_{p}+\|\boldsymbol{f}\|_{p, B}\right)^{p-2}\|\boldsymbol{g}\|_{p, B}
$$

which gives (5.5). Estimates (5.7) and (5.8) are a direct consequence of the coercivity properties and the monotonicity properties in Lemma 3.1. Finally, observing that

$$
\begin{aligned}
\|\boldsymbol{\tau}(\boldsymbol{f})-\boldsymbol{\tau}(\boldsymbol{g})\|_{p^{\prime}, B} & \leq(p-1)\left\|\left(1+|\boldsymbol{f}|^{2}+|\boldsymbol{g}|^{2}\right)^{\frac{p-2}{2}}|\boldsymbol{f}-\boldsymbol{g}|\right\|_{p^{\prime}, B} \\
& \leq(p-1)\left\|\left(1+|\boldsymbol{f}|^{2}+|\boldsymbol{g}|^{2}\right)^{\frac{p-2}{2}}\right\|\left\|_{\frac{p}{p-2}, B}\right\| \boldsymbol{f}-\boldsymbol{g} \|_{p, B} \\
& \leq(p-1)\left(\left\|B^{\frac{1}{p}}\right\|_{p}+\|\boldsymbol{f}\|_{p, B}+\|\boldsymbol{g}\|_{p, B}\right)^{p-2}\|\boldsymbol{f}-\boldsymbol{g}\|_{p, B}
\end{aligned}
$$

we obtain (5.6). The case $p=2$ is direct.

We finish the section by a result that will be useful in the sequel.

Proposition 5.6 Assume that $p \geq 2$ and let $\boldsymbol{f} \in \boldsymbol{L}^{p}\left(\Sigma, \mathbb{R}^{3 \times 3}\right)$. Then the following estimate holds

$$
\left\|\nabla^{\star} \cdot \boldsymbol{\tau}(\boldsymbol{f})-\nabla \cdot \boldsymbol{\tau}(\boldsymbol{f})\right\|_{p^{\prime}} \leq 4 \delta m \mathcal{F}_{1}\left(\|\boldsymbol{f}\|_{p}\right)\left(\|f\|_{p}+\left\|f_{33}\right\|_{p}+\left\|f_{23}\right\|_{p}\right)
$$

where $\mathcal{F}_{1}$ is defined in Proposition 5.5 and $f=\left(f_{i j}\right)_{i, j=1,2}$.

Proof. Taking into account the definition of $\nabla^{\star}$, we obtain

$$
\begin{aligned}
\left\|\nabla^{\star} \cdot \boldsymbol{\tau}(\boldsymbol{f})-\nabla \cdot \boldsymbol{\tau}(\boldsymbol{f})\right\|_{p^{\prime}} & =\left\|\frac{\delta}{B}\left(\tau_{12}(\boldsymbol{f}) \boldsymbol{a}_{1}+\left(\tau_{22}(\boldsymbol{f})-\tau_{33}(\boldsymbol{f})\right) \boldsymbol{a}_{2}+2 \tau_{23}(\boldsymbol{f}) \boldsymbol{a}_{3}\right)\right\|_{p^{\prime}} \\
& \leq \delta m\left(\left\|\tau_{12}(\boldsymbol{f})\right\|_{p^{\prime}}+\left\|\tau_{22}(\boldsymbol{f})\right\|_{p^{\prime}}+\left\|\tau_{33}(\boldsymbol{f})\right\|_{p^{\prime}}+2\left\|\tau_{23}(\boldsymbol{f})\right\|_{p^{\prime}}\right) \\
& \leq 2 \delta m\left(\|\tau(\boldsymbol{f})\|_{p^{\prime}}+\left\|\tau_{33}(\boldsymbol{f})\right\|_{p^{\prime}}+\left\|\tau_{23}(\boldsymbol{f})\right\|_{p^{\prime}}\right)
\end{aligned}
$$

and the conclusion follows from estimate (5.5) . 


\subsection{Existence and uniqueness of shear-thickening flows}

The aim of this section is to prove the existence and uniqueness result Theorem 4.2 As usual, we first derive some estimates that hold not only for the exact solution $\boldsymbol{u}$ of (4.1), but also for corresponding standard Galerkin approximations $\boldsymbol{u}^{k}$.

Proposition 5.7 Assume that $p \geq 2$ and let $\boldsymbol{u}$ be a weak solution of (4.1). Then, estimates (4.4)-(4.8) hold.

Proof. The proof is split into two steps.

Step 1. Global estimates. Setting $\boldsymbol{\varphi}=\boldsymbol{u}$ in (4.1), and using Lemma 5.3 and estimate (5.7), we deduce that

$$
\begin{aligned}
& 2\left\|D^{\star} \boldsymbol{u}\right\|_{2, B}^{2} \leq\left(\boldsymbol{\tau}\left(D^{\star} \boldsymbol{u}\right), B D^{\star} \boldsymbol{u}\right)=\left(G, u_{3}\right), \\
& 2\left\|D^{\star} \boldsymbol{u}\right\|_{p, B}^{p} \leq\left(\boldsymbol{\tau}\left(D^{\star} \boldsymbol{u}\right), B D^{\star} \boldsymbol{u}\right)=\left(G, u_{3}\right) .
\end{aligned}
$$

Classical arguments together with (3.8) and (5.2) yield

$$
\begin{aligned}
\left(G, u_{3}\right) & =\left|\left(\frac{G}{\sqrt{B}}, \sqrt{B} u_{3}\right)\right| \\
& \leq|G|\left\|\frac{1}{\sqrt{B}}\right\|_{2}\left\|u_{3}\right\|_{2, B}=|G|\left\|B^{-1}\right\|_{1}^{\frac{1}{2}}\left\|u_{3}\right\|_{2, B} \\
& \leq \kappa_{1}\left\|\nabla\left(\sqrt{B} u_{3}\right)\right\|_{2} \leq \sqrt{2} \kappa_{1}\left(\left\|\nabla u_{3}\right\|_{2, B}^{2}+\left\|\frac{\delta}{B} u_{3}\right\|_{2, B}^{2}\right)^{\frac{1}{2}} \\
& \leq 2 \kappa_{1}\left\|D^{\star} \boldsymbol{u}\right\|_{2, B},
\end{aligned}
$$

where $\kappa_{1}=\left(\frac{m}{2}\right)^{\frac{1}{2}}|G||\Sigma|$. Due to (5.10) and (15.12), we obtain (4.4) and

$$
\left(G, u_{3}\right) \leq 2 \kappa_{1}^{2}
$$

Estimate (4.5) follows then from (5.11) and (5.13).

Step 2. Estimates for $u$ and $u_{3}$. Let us now prove estimates (4.6)-(4.8). Notice first that (4.6) is a direct consequence of (4.4) and (5.2). To derive the second estimate, we set $\varphi=u$ in the weak formulation (4.2) and get

$$
\left(\tau\left(D^{\star} \boldsymbol{u}\right), B D u\right)+\delta\left(\tau_{33}\left(D^{\star} \boldsymbol{u}\right), u_{2}\right)=\delta \mathcal{R} e\left(u_{3}^{2}, u_{2}\right) .
$$

Therefore, by using the coercivity properties, we obtain

$$
\begin{aligned}
& \|D u\|_{2, B}^{2}+\left\|\frac{\delta}{B} u_{2}\right\|_{2, B}^{2} \leq \frac{\delta \mathcal{R} e}{2}\left(u_{3}^{2}, u_{2}\right), \\
& \|D u\|_{p, B}^{p}+\left\|\frac{\delta}{B} u_{2}\right\|_{p, B}^{p} \leq \frac{\delta \mathcal{R} e}{2}\left(u_{3}^{2}, u_{2}\right) .
\end{aligned}
$$

On the other hand, taking into account (3.13) with $\alpha=q=2,(5.3)$ and (4.6), we have

$$
\begin{aligned}
\left|\left(u_{3}^{2}, u_{2}\right)\right| & \leq\left(S_{2,4}\right)^{3}|\Sigma|^{\frac{1}{4}}\left\|\nabla u_{3}\right\|_{2}^{2}\left\|\nabla u_{2}\right\|_{2} \\
& \leq \frac{m^{\frac{3}{2}}|\Sigma|}{2 \sqrt{2}}\left\|\nabla u_{3}\right\|_{2, B}^{2}\left\|\nabla u_{2}\right\|_{2, B} \\
& \leq \frac{m^{\frac{3}{2}}|\Sigma|}{2}\left\|\nabla u_{3}\right\|_{2, B}^{2}\|D u\|_{2, B} \\
& \leq m^{\frac{3}{2}}|\Sigma| \kappa_{1}^{2}\|D u\|_{2, B} .
\end{aligned}
$$


Combining (5.14) and (5.16), we obtain (4.7) and

$$
\left|\left(u_{3}^{2}, u_{2}\right)\right| \leq 2\left(\frac{m^{\frac{3}{2}}|\Sigma| \kappa_{1}^{2}}{2}\right)^{2} \delta \mathcal{R} e .
$$

Estimate (4.8) follows then from (5.15).

Proof of Theorem 4.2, The proof, based on classical compactness and monotonicity arguments, is split into two steps.

Step 1. Let us prove the existence of a weak solution for (2.7). Let $\boldsymbol{u}^{k}$ be a classical Galerkin approximation. Arguments similar to those used in the proof of Proposition 5.7 show that

$$
\left\|D^{\star} \boldsymbol{u}^{k}\right\|_{2, B} \leq \kappa_{1}, \quad\left\|D^{\star} \boldsymbol{u}^{k}\right\|_{p, B}^{p} \leq \kappa_{1}^{2}
$$

and imply that the sequences $\left(\sqrt{B} D^{\star} \boldsymbol{u}^{k}\right)_{k}$ and $\left(B^{\frac{1}{p}} D^{\star} \boldsymbol{u}^{k}\right)_{k}$ are bounded in $\boldsymbol{L}^{2}(\Sigma)$ and $\boldsymbol{L}^{p}(\Sigma)$, respectively. By taking into account (5.4), we deduce that $\left(\sqrt{B} \nabla \boldsymbol{u}^{k}\right)_{k}$ is bounded in $\boldsymbol{L}^{2}(\Sigma)$ and thus $\left(\nabla \boldsymbol{u}^{k}\right)_{k}$ is also bounded in $\boldsymbol{L}^{2}(\Sigma)$. Moreover, due to estimate (5.5) we have

$$
\left\|\boldsymbol{\tau}\left(D^{\star} \boldsymbol{u}^{k}\right)\right\|_{p^{\prime}, B} \leq 2 \mathcal{F}_{B}\left(\left\|D^{\star} \boldsymbol{u}^{k}\right\|_{p, B}\right)\left\|D^{\star} \boldsymbol{u}^{k}\right\|_{p, B}
$$

and thus $\left(B^{\frac{1}{p^{\prime}} \boldsymbol{\tau}}\left(D^{\star} \boldsymbol{u}^{k}\right)\right)_{k}$ is bounded in $\boldsymbol{L}^{p^{\prime}}(\Sigma)$. There then exist a subsequence, still indexed by $k, \boldsymbol{u} \in \boldsymbol{V}_{B}^{p}$ and $\tilde{\boldsymbol{\tau}} \in \boldsymbol{L}^{p^{\prime}}(\Sigma)$ such that

$$
\begin{gathered}
\nabla \boldsymbol{u}^{k} \longrightarrow \nabla \boldsymbol{u} \quad \text { weakly in } \boldsymbol{L}^{2}(\Sigma), \\
B^{\frac{1}{p^{\prime}} \boldsymbol{\tau}\left(D^{\star} \boldsymbol{u}^{k}\right) \longrightarrow \widetilde{\boldsymbol{\tau}}} \quad \text { weakly in } \boldsymbol{L}^{p^{\prime}}(\Sigma) .
\end{gathered}
$$

By using compactness results on Sobolev spaces, we deduce that $\left(\boldsymbol{u}^{k}\right)_{k}$ strongly converges to $\boldsymbol{u}$ in $L^{4}(\Sigma)$ and thus

$$
\sqrt{B} D^{\star} \boldsymbol{u}^{k} \longrightarrow \sqrt{B} D^{\star} \boldsymbol{u} \quad \text { weakly in } \boldsymbol{L}^{2}(\Sigma) \text {. }
$$

Therefore, by taking into account Lemma 5.3 , for all $\varphi \in \boldsymbol{V}_{B}^{p}$ we have

$$
\begin{aligned}
\left|a_{\star}\left(\boldsymbol{u}^{k}, \boldsymbol{u}^{k}, B \boldsymbol{\varphi}\right)-a_{\star}(\boldsymbol{u}, \boldsymbol{u}, B \boldsymbol{\varphi})\right| & \leq\left|a_{\star}\left(\boldsymbol{u}^{k}-\boldsymbol{u}, \boldsymbol{u}^{k}, B \boldsymbol{\varphi}\right)\right|+\left|a_{\star}\left(\boldsymbol{u}, \boldsymbol{u}^{k}-\boldsymbol{u}, B \boldsymbol{\varphi}\right)\right| \\
& =\left|a_{\star}\left(\boldsymbol{u}^{k}-\boldsymbol{u}, \boldsymbol{u}^{k}, B \boldsymbol{\varphi}\right)\right|+\left|a_{\star}\left(B \boldsymbol{u}, \boldsymbol{\varphi}, \boldsymbol{u}^{k}-\boldsymbol{u}\right)\right| \\
& \leq\left(\left\|\nabla^{\star} \boldsymbol{u}^{k}\right\|_{2, B}\|\boldsymbol{\varphi}\|_{4, B}+\|\boldsymbol{u}\|_{4, B}\left\|\nabla^{\star} \boldsymbol{\varphi}\right\|_{2, B}\right)\left\|\boldsymbol{u}^{k}-\boldsymbol{u}\right\|_{4, B} \\
& \longrightarrow 0 \quad \text { when } k \rightarrow+\infty .
\end{aligned}
$$

By passing to the limit in

$$
\left(\boldsymbol{\tau}\left(D^{\star} \boldsymbol{u}^{k}\right), B D^{\star} \boldsymbol{\varphi}\right)+\mathcal{R} e a_{\star}\left(\boldsymbol{u}^{k}, \boldsymbol{u}^{k}, B \boldsymbol{\varphi}\right)=\left(G, \varphi_{3}\right) \quad \text { for all } \boldsymbol{\varphi} \in \boldsymbol{V}_{B}^{p},
$$

we obtain

$$
\left(\widetilde{\boldsymbol{\tau}}, B^{\frac{1}{p}} D^{\star} \boldsymbol{\varphi}\right)+\mathcal{R} e a_{\star}(\boldsymbol{u}, \boldsymbol{u}, B \boldsymbol{\varphi})=\left(G, \varphi_{3}\right) \quad \text { for all } \boldsymbol{\varphi} \in \boldsymbol{V}_{B}^{p} .
$$

In particular, by settin $\varphi=\boldsymbol{u}$ and using Lemma $[5.3$ we deduce that

$$
\left(\widetilde{\boldsymbol{\tau}}, B^{\frac{1}{p}} D^{\star} \boldsymbol{u}\right)=\left(G, u_{3}\right) .
$$

On the other hand, $(\overline{5.8})_{1}$ implies

$$
\left(\boldsymbol{\tau}\left(D^{\star} \boldsymbol{u}^{k}\right)-\boldsymbol{\tau}\left(D^{\star} \boldsymbol{\varphi}\right), B D^{\star}\left(\boldsymbol{u}^{k}-\boldsymbol{\varphi}\right)\right) \geq 0 \quad \text { for all } \boldsymbol{\varphi} \in \boldsymbol{V}_{B}^{p} .
$$


Since

$$
\left(\boldsymbol{\tau}\left(D^{\star} \boldsymbol{u}^{k}\right), B D^{\star} \boldsymbol{u}^{k}\right)=\left(G, u_{3}^{k}\right),
$$

by substituing in (5.19), we obtain

$$
\left(G, u_{3}^{k}\right)-\left(\boldsymbol{\tau}\left(D^{\star} \boldsymbol{u}^{k}\right), B D^{\star} \boldsymbol{\varphi}\right)-\left(\boldsymbol{\tau}\left(D^{\star} \boldsymbol{\varphi}\right), B D^{\star}\left(\boldsymbol{u}^{k}-\boldsymbol{\varphi}\right)\right) \geq 0 .
$$

By passing to the limit, it follows that

$$
\left(G, u_{3}\right)-\left(B^{\frac{1}{p}} \widetilde{\boldsymbol{\tau}}, D^{\star} \boldsymbol{\varphi}\right)-\left(B \boldsymbol{\tau}\left(D^{\star} \boldsymbol{\varphi}\right), D^{\star}(\boldsymbol{u}-\boldsymbol{\varphi})\right) \geq 0 \quad \text { for all } \boldsymbol{\varphi} \in \boldsymbol{V}_{B}^{p} .
$$

This inequality together with (5.18) implies that

$$
\left(B^{\frac{1}{p}} \widetilde{\boldsymbol{\tau}}-B \boldsymbol{\tau}\left(D^{\star} \boldsymbol{\varphi}\right), D^{\star}(\boldsymbol{u}-\boldsymbol{\varphi})\right) \geq 0 \quad \text { for all } \boldsymbol{\varphi} \in \boldsymbol{V}_{B}^{p}
$$

and by setting $\boldsymbol{\varphi}=\boldsymbol{u}-t \boldsymbol{\psi}$ with $t>0$, we obtain

$$
\left(B^{\frac{1}{p}} \widetilde{\boldsymbol{\tau}}-B \boldsymbol{\tau}\left(D^{\star} \boldsymbol{u}-t D \boldsymbol{\psi}\right), D^{\star} \boldsymbol{\psi}\right) \geq 0 \quad \text { for all } \boldsymbol{\psi} \in \boldsymbol{V}_{B}^{p} .
$$

Letting $t$ tend to zero and using the continuity of $\tau$, we get

$$
\left(B^{\frac{1}{p}} \widetilde{\boldsymbol{\tau}}-B \boldsymbol{\tau}\left(D^{\star} \boldsymbol{u}\right), D^{\star} \boldsymbol{\psi}\right) \geq 0 \quad \text { for all } \boldsymbol{\psi} \in \boldsymbol{V}_{B}^{p}
$$

and thus

$$
\left(B^{\frac{1}{p}} \widetilde{\boldsymbol{\tau}}, D^{\star} \boldsymbol{\psi}\right)=\left(B \boldsymbol{\tau}\left(D^{\star} \boldsymbol{u}\right), D^{\star} \boldsymbol{\psi}\right) \quad \text { for all } \boldsymbol{\psi} \in \boldsymbol{V}_{B}^{p} .
$$

Combining (5.17) and (5.20), we deduce that

$$
\left(B \boldsymbol{\tau}\left(D^{\star} \boldsymbol{u}\right), D^{\star} \boldsymbol{\varphi}\right)+\mathcal{R} e a_{\star}(\boldsymbol{u}, \boldsymbol{u}, B \boldsymbol{\varphi})=\left(G, \varphi_{3}\right) \quad \text { for all } \boldsymbol{\varphi} \in \boldsymbol{V}_{B}^{p} .
$$

Hence $\boldsymbol{u}$ is a solution of (4.1)

Step 2. To prove the uniqueness result, let us assume that $\boldsymbol{u}$ and $\boldsymbol{v}$ are two weak solutions of (2.7). Substituting in the weak formulation of (4.1), setting $\boldsymbol{\varphi}=\boldsymbol{u}-\boldsymbol{v}$ and taking into account Lemma [5.3, Lemma 5.4 and Proposition 5.7, we obtain

$$
\begin{aligned}
\frac{1}{\mathcal{R} e}\left(\boldsymbol{\tau}\left(D^{\star} \boldsymbol{u}\right)-\boldsymbol{\tau}\left(D^{\star} \boldsymbol{v}\right), B D^{\star}(\boldsymbol{u}-\boldsymbol{v})\right) & =-a_{\star}(\boldsymbol{u}, \boldsymbol{u}, B(\boldsymbol{u}-\boldsymbol{v}))+a_{\star}(\boldsymbol{v}, \boldsymbol{v}, B(\boldsymbol{u}-\boldsymbol{v})) \\
& =-a_{\star}(\boldsymbol{u}, \boldsymbol{u}-\boldsymbol{v}, B(\boldsymbol{u}-\boldsymbol{v}))-a_{\star}(\boldsymbol{u}-\boldsymbol{v}, \boldsymbol{v}, B(\boldsymbol{u}-\boldsymbol{v})) \\
& =-a_{\star}(\boldsymbol{u}-\boldsymbol{v}, \boldsymbol{v}, B(\boldsymbol{u}-\boldsymbol{v})) \\
& \leq \kappa_{3}\left\|D^{\star}(\boldsymbol{u}-\boldsymbol{v})\right\|_{2, B}^{2}\left\|D^{\star} \boldsymbol{v}\right\|_{2, B} \\
& \leq \kappa_{1} \kappa_{3}\left\|D^{\star}(\boldsymbol{u}-\boldsymbol{v})\right\|_{2, B}^{2} .
\end{aligned}
$$

Combining (5.21) and (5.7) 1 , we deduce that

$$
\left(2-\kappa_{1} \kappa_{3} \mathcal{R} e\right)\left\|D^{\star}(\boldsymbol{u}-\boldsymbol{v})\right\|_{2, B}^{2} \leq 0
$$

and thus $\boldsymbol{u} \equiv \boldsymbol{v}$ if $\mathcal{R} e<\frac{2}{\kappa_{1} \kappa_{3}}$.

Proof of Corollary 4.5. To simplify the redaction, let us set $\boldsymbol{\tau}\left(D^{\star} \boldsymbol{u}\right)=\boldsymbol{\tau}$. Notice first that if $\boldsymbol{u}$ is a weak solution of (2.7) then

$$
(\boldsymbol{\tau}, D \boldsymbol{\varphi})-\left(\nabla^{\star} \cdot \boldsymbol{\tau}-\nabla \cdot \boldsymbol{\tau}, \boldsymbol{\varphi}\right)+\mathcal{R} e a_{\star}(\boldsymbol{u}, \boldsymbol{u}, \boldsymbol{\varphi})=\left(\frac{G}{B}, \varphi_{3}\right) \quad \text { for all } \boldsymbol{\varphi} \in \boldsymbol{V}^{p} .
$$


It follows that $u=\left(u_{1}, u_{2}, 0\right)$ satisfies

$$
(\tau, D \varphi)-\left(\nabla^{\star} \cdot \tau-\nabla \cdot \tau, \varphi\right)+\mathcal{R} e\left((u \cdot \nabla u, \varphi)-\left(\frac{\delta}{B} u_{3}^{2}, \varphi_{2}\right)\right)=0
$$

for all $\varphi=\left(\varphi_{1}, \varphi_{2}\right) \in \boldsymbol{W}_{0}^{1, p}\left(\Sigma, \mathbb{R}^{2}\right)$ such that $\nabla \cdot \varphi=0$, with $\tau=\left(\tau_{i j}\right)_{i, j=1,2}$. Taking into account (5.5), (5.9) and using standard arguments, we can prove that the mapping

$$
\mathcal{G}: \varphi \mapsto(\tau, D \varphi)-\left(\nabla^{\star} \cdot \tau-\nabla \cdot \tau, \varphi\right)+\mathcal{R} e\left((u \cdot \nabla u, \varphi)-\left(\frac{\delta}{B} u_{3}^{2}, \varphi_{2}\right)\right)
$$

is a linear continuous functional on $\boldsymbol{W}_{0}^{1, p}\left(\Sigma, \mathbb{R}^{2}\right)$. By using a classical result (see [1]), we deduce that there exists $\pi \in \boldsymbol{L}_{0}^{p^{\prime}}(\Sigma)$ such that

$$
\mathcal{G}(\varphi)=-(\nabla \pi, \varphi)=(\pi, \nabla \cdot \varphi) \quad \text { for all } \varphi \in \boldsymbol{W}_{0}^{1, p}\left(\Sigma, \mathbb{R}^{2}\right) .
$$

Moreover, there exists a positive constant $C$ depending only on $p$ and $\Sigma$ such that

$$
C\|\pi\|_{p^{\prime}} \leq\|\nabla \pi\|_{-1, p^{\prime}}=\sup _{\varphi \in \boldsymbol{W}_{0}^{1, p}\left(\Sigma, \mathbb{R}^{2}\right)} \frac{|(\pi, \nabla \cdot \varphi)|}{\|\nabla \varphi\|_{p}} .
$$

On the other hand, using (3.8), (5.5), (5.9) and (4.5) we obtain

$$
\begin{aligned}
\left|(\tau, D \varphi)-\left(\nabla^{\star} \cdot \tau-\nabla \cdot \tau, \varphi\right)\right| & \leq\|\tau\|_{p^{\prime}}\|D \varphi\|_{p}+\left\|\nabla^{\star} \cdot \tau-\nabla \cdot \tau\right\|_{p^{\prime}}\|\varphi\|_{p} \\
& \leq\left(\|\tau\|_{p^{\prime}}+S_{p, p}\left\|\nabla^{\star} \cdot \tau-\nabla \cdot \tau\right\|_{p^{\prime}}\right)\|\nabla \varphi\|_{p} \\
& \leq \mathcal{F}_{1}\left(\left\|D^{\star} \boldsymbol{u}\right\|_{p}\right)\left(\left(1+4 S_{p, p} \delta m\right)\|D u\|_{p}+4 S_{p, p} \delta m\left\|\frac{\delta}{B} u_{2}\right\|_{p}\right)\|\nabla \varphi\|_{p} \\
& \leq \tilde{\kappa}\left(\|D u\|_{p, B}+\delta^{2}\left\|u_{2}\right\|_{p, B}\right)\|\nabla \varphi\|_{p},
\end{aligned}
$$

where $\tilde{\kappa}$ only depends on $p, \Sigma, m$ and $n$. Similarly, we can easily see that

$$
\begin{aligned}
\left|(u \cdot \nabla u, \varphi)-\left(\frac{\delta}{B} u_{3}^{2}, \varphi_{2}\right)\right| & =\left|-(u \otimes u, \nabla \varphi)-\left(\frac{\delta}{B} u_{3}^{2}, \varphi_{2}\right)\right| \\
& \leq\|u \otimes u\|_{p^{\prime}}\|\nabla \varphi\|_{p}+\delta m\left\|u_{3}^{2}\right\|_{p^{\prime}}\left\|\varphi_{2}\right\|_{p} \\
& \leq \hat{\kappa}\left(\|D u\|_{p, B}^{2}+\delta\left\|\nabla u_{3}\right\|_{p, B}^{2}\right)\|\nabla \varphi\|_{p},
\end{aligned}
$$

where $\hat{\kappa}$ only depends on $p, \Sigma$ and $m$. Combining (5.23)-(5.25), we deduce that

$$
\|\pi\|_{p^{\prime}} \leq \kappa\left(\|D u\|_{p, B}+\delta^{2}\left\|u_{2}\right\|_{p, B}+\mathcal{R} e\left(\|D u\|_{p, B}^{2}+\delta\left\|\nabla u_{3}\right\|_{p, B}^{2}\right)\right),
$$

where $\kappa$ is a positive constant only depending on $\Sigma, p, m$ and $n$.

\section{$5.5 \delta$-approximation}

Proof of Proposition 4.9, Based on a standard Galerkin approximation of the corresponding global formulation, compactness and monotonicity arguments, the existence of a weak solution for $\left(E_{\sigma}\right)$ can be established once suitable a priori estimates are derived. However, because of the term involving $\sigma\left(w_{3}\right)$, the global formulation does not seem appropriate unless we restrict strongly the exponent $\alpha$ in (3.12). To overcome this difficulty, we consider the coupled formulations. Arguing as in the proof of Proposition 5.7, by setting $\varphi=\left(0,0, w_{3}\right)$ in the corresponding weak formulation we obtain

$$
\int_{\Sigma}\left(1+|D \boldsymbol{w}|^{2}\right)^{\frac{p-2}{2}}\left|\nabla w_{3}\right|^{2} d x=\left(\frac{G}{B}, w_{3}\right)
$$


and thus

$$
\left\|\nabla w_{3}\right\|_{2}^{2} \leq\left(\frac{G}{B}, w_{3}\right) \leq c_{1}\left\|\nabla w_{3}\right\|_{2} \quad \text { with } c_{1}=\frac{m|G||\Sigma|}{\sqrt{2}}
$$

Hence

$$
\left\|\nabla w_{3}\right\|_{2} \leq c_{1}, \quad\left(\frac{G}{B}, w_{3}\right) \leq c_{1}^{2}
$$

and

$$
\left(\frac{1}{2}\right)^{\frac{p-2}{2}}\left\|\nabla w_{3}\right\|_{p}^{p} \leq\left(\frac{G}{B}, w_{3}\right) \leq c_{1}^{2} .
$$

Similarly, by setting $\boldsymbol{\varphi}=(w, 0)=\left(w_{1}, w_{2}, 0\right)$ in the corresponding weak formulation, we obtain

$$
2 \int_{\Sigma}\left(1+|D \boldsymbol{w}|^{2}\right)^{\frac{p-2}{2}}|D w|^{2} d x=\delta\left(\sigma\left(w_{3}\right), w_{2}\right)
$$

and thus

$$
\|D w\|_{2}^{2} \leq \frac{\delta}{2}\left(\sigma\left(w_{3}\right), w_{2}\right), \quad\|D w\|_{p}^{p} \leq \frac{\delta}{2}\left(\sigma\left(w_{3}\right), w_{2}\right) .
$$

Estimate (3.13) together with the Korn inequality yield

$$
\|D w\|_{2}^{2} \leq \frac{c_{0} D_{2, \alpha}}{2} \delta\left\|\nabla w_{3}\right\|_{2}^{\alpha}\left\|\nabla w_{2}\right\|_{2} \leq \frac{c_{0} D_{2, \alpha}}{2} \delta\left\|\nabla w_{3}\right\|_{2}^{\alpha}\|\nabla w\|_{2} \leq c_{0} c_{2} \delta\|D w\|_{2}
$$

and consequently

$$
\|D w\|_{2} \leq c_{0} c_{2} \delta, \quad\left(\sigma\left(w_{3}\right), w_{2}\right) \leq 2 \delta\left(c_{0} c_{2}\right)^{2}
$$

Therefore

$$
\|D w\|_{p}^{p} \leq\left(c_{0} c_{2} \delta\right)^{2}
$$

and the a priori estimates are derived. The proof may be completed using arguments similar to those in the proof of Theorem 4.2 .

Remark 5.8 Arguing as in the proof of Corollary 4.5 and using (3.13), we can prove the existence of $\pi \in L^{p^{\prime}}(\Sigma)$ such that $\left(E_{\sigma}\right)_{1}$ holds in $\boldsymbol{W}^{-1, p^{\prime}}(\Sigma)$. Moreover, the following estimate holds

$$
\|\pi\|_{p^{\prime}} \leq \kappa\left(\|D w\|_{p}+\mathcal{R} e\|D w\|_{p}^{2}+\delta\left\|\nabla w_{3}\right\|_{p}^{\alpha}\right)
$$

where $\kappa$ is a positive constant only depending on $p, \Sigma, m, n$ and $\alpha$.

Proof of Proposition 4.10, Let us first recall that $\boldsymbol{u}$ satisfies (5.22) and that $\boldsymbol{w}$ satisfies

$$
(\boldsymbol{\tau}(D \boldsymbol{w}), D \boldsymbol{\varphi})+\mathcal{R} e a(\boldsymbol{w}, \boldsymbol{w}, \boldsymbol{\varphi})-\left(\pi_{2}, \nabla \cdot \boldsymbol{\varphi}\right)=\left(\frac{G}{B}, \varphi_{3}\right)+\delta\left(\sigma\left(w_{3}\right), \varphi_{2}\right)
$$

for all $\varphi \in \boldsymbol{W}_{0}^{1, p}(\Sigma)$. Therefore

$$
\begin{aligned}
(\boldsymbol{\tau}(D \boldsymbol{u})-\boldsymbol{\tau}(D \boldsymbol{w}), D \boldsymbol{\varphi}) & =\left(\boldsymbol{\tau}(D \boldsymbol{u})-\boldsymbol{\tau}\left(D^{\star} \boldsymbol{u}\right), D \boldsymbol{\varphi}\right)+\left(\nabla^{\star} \cdot \boldsymbol{\tau}\left(D^{\star} \boldsymbol{u}\right)-\nabla \cdot \boldsymbol{\tau}\left(D^{\star} \boldsymbol{u}\right), \boldsymbol{\varphi}\right) \\
& -\mathcal{R} e\left(a_{\star}(\boldsymbol{u}, \boldsymbol{u}, \boldsymbol{\varphi})-a(\boldsymbol{w}, \boldsymbol{w}, \boldsymbol{\varphi})\right)+\left(\pi_{1}-\pi_{2}, \nabla \cdot \boldsymbol{\varphi}\right)-\delta\left(\sigma\left(w_{3}\right), \varphi_{2}\right) \\
& =I_{1}+I_{2}+I_{3}+I_{4}+I_{5} .
\end{aligned}
$$

○ Let us estimate the first term. By taking into account (5.6), we have

$$
\begin{aligned}
\left|I_{1}\right| & =\left|\left(\boldsymbol{\tau}\left(D^{\star} \boldsymbol{u}\right)-\boldsymbol{\tau}(D \boldsymbol{u}), D \boldsymbol{\varphi}\right)\right| \leq\left\|\boldsymbol{\tau}\left(D^{\star} \boldsymbol{u}\right)-\boldsymbol{\tau}(D \boldsymbol{u})\right\|_{p^{\prime}}\|D \boldsymbol{\varphi}\|_{p} \\
& \leq(p-1) \mathcal{F}_{1}\left(\left\|D^{\star} \boldsymbol{u}\right\|_{p}+\|D \boldsymbol{u}\|_{p}\right)\left\|D^{\star} \boldsymbol{u}-D \boldsymbol{u}\right\|_{p}\|D \boldsymbol{\varphi}\|_{p}
\end{aligned}
$$

Since

$$
\left\|D^{\star} \boldsymbol{u}-D \boldsymbol{u}\right\|_{p} \leq \delta m\left(\left\|u_{2}\right\|_{p}+\left\|u_{3}\right\|_{p}\right),
$$


we deduce that

$$
\left|I_{1}\right| \leq F_{1} \delta\|D \varphi\|_{p}
$$

where $F_{1}=m(p-1) \mathcal{F}_{1}\left(\left\|D^{\star} \boldsymbol{u}\right\|_{p}+\|D \boldsymbol{u}\|_{p}\right)\left(\left\|u_{2}\right\|_{p}+\left\|u_{3}\right\|_{p}\right)$.

$\circ$ Estimate (5.9) together with the Sobolev inequality (3.8) and the Korn inequality (5.1) yield

$$
\begin{aligned}
\left|I_{2}\right| & \leq\left\|\nabla^{\star} \cdot \boldsymbol{\tau}\left(D^{\star} \boldsymbol{u}\right)-\nabla \cdot \boldsymbol{\tau}\left(D^{\star} \boldsymbol{u}\right)\right\|_{p^{\prime}}\|\boldsymbol{\varphi}\|_{p} \\
& \leq 4 S_{2, p} m \mathcal{F}_{1}\left(\left\|D^{\star} \boldsymbol{u}\right\|_{p}\right)\left(\|D u\|_{p}+\left\|D_{33}^{\star} \boldsymbol{u}\right\|_{p}+\left\|D_{23}^{\star} \boldsymbol{u}\right\|_{p}\right) \delta\|\nabla \boldsymbol{\varphi}\|_{2} \leq F_{2} \delta\|D \boldsymbol{\varphi}\|_{p},
\end{aligned}
$$

where $F_{2}=4 \sqrt{6} m|\Sigma|^{\frac{1}{2}-\frac{1}{p}} S_{2, p} \mathcal{F}_{1}\left(\left\|D^{\star} \boldsymbol{u}\right\|_{p}\right)\left\|D^{\star} \boldsymbol{u}\right\|_{p}$.

○ Similarly,

$$
\begin{aligned}
\frac{1}{\mathcal{R} e}\left|I_{3}\right| & =\left|a_{\star}(\boldsymbol{u}, \boldsymbol{u}, \boldsymbol{\varphi})-a(\boldsymbol{w}, \boldsymbol{w}, \boldsymbol{\varphi})\right| \\
& =\left|a_{\star}(\boldsymbol{u}-\boldsymbol{w}, \boldsymbol{u}, \boldsymbol{\varphi})+a(\boldsymbol{w}, \boldsymbol{u}-\boldsymbol{w}, \boldsymbol{\varphi})+\left(\boldsymbol{w} \cdot \nabla^{\star} \boldsymbol{u}-\boldsymbol{w} \cdot \nabla \boldsymbol{u}, \boldsymbol{\varphi}\right)\right| \\
& \leq\left|a_{\star}(\boldsymbol{u}-\boldsymbol{w}, \boldsymbol{u}, \boldsymbol{\varphi})\right|+|a(\boldsymbol{w}, \boldsymbol{u}-\boldsymbol{w}, \boldsymbol{\varphi})|+\left|\left(\boldsymbol{w} \cdot \nabla^{\star} \boldsymbol{u}-\boldsymbol{w} \cdot \nabla \boldsymbol{u}, \boldsymbol{\varphi}\right)\right| \\
& =\left|a_{\star}(\boldsymbol{u}-\boldsymbol{w}, \boldsymbol{u}, \boldsymbol{\varphi})\right|+|a(\boldsymbol{w}, \boldsymbol{u}-\boldsymbol{w}, \boldsymbol{\varphi})|+\left|\left(\frac{\delta}{B} w_{2} u_{2}, \varphi_{3}\right)-\left(\frac{\delta}{B} w_{3} u_{3}, \varphi_{2}\right)\right| \\
& \leq\left|a_{\star}(\boldsymbol{u}-\boldsymbol{w}, \boldsymbol{u}, \boldsymbol{\varphi})\right|+|a(\boldsymbol{w}, \boldsymbol{u}-\boldsymbol{w}, \boldsymbol{\varphi})|+\left\|\frac{\delta}{B} \mid \boldsymbol{w}\right\| \boldsymbol{u}\|\boldsymbol{\varphi}\|_{1} \\
& \leq\|\boldsymbol{u}-\boldsymbol{w}\|_{4}\left\|\nabla^{\star} \boldsymbol{u}\right\|_{2}\|\boldsymbol{\varphi}\|_{4}+\|\boldsymbol{w}\|_{4}\|\nabla(\boldsymbol{u}-\boldsymbol{w})\|_{2}\|\boldsymbol{\varphi}\|_{4}+\delta m\|\boldsymbol{w}\|_{4}\|\boldsymbol{u}\|_{4}\|\boldsymbol{\varphi}\|_{2} \\
& \leq\left(1+S_{2,2}\right)\left(S_{2,4}\right)^{2}\left(\|\nabla(\boldsymbol{u}-\boldsymbol{w})\|_{2}\left(\left\|\nabla^{\star} \boldsymbol{u}\right\|_{2}+\|\nabla \boldsymbol{w}\|_{2}\right)+\delta m\|\nabla \boldsymbol{w}\|_{2}\|\nabla \boldsymbol{u}\|_{2}\right)\|\nabla \boldsymbol{\varphi}\|_{2} \\
& \leq F_{3}\left(\|D(\boldsymbol{u}-\boldsymbol{w})\|_{2}\|D \boldsymbol{\varphi}\|_{2}+\delta\|D \boldsymbol{\varphi}\|_{p}\right)
\end{aligned}
$$

with $F_{3}=(1+m) \sqrt{8}\left(1+S_{2,2}\right)\left(S_{2,4}\right)^{2}\left(\left\|D^{\star} \boldsymbol{u}\right\|_{2}+\|D \boldsymbol{w}\|_{2}+\|D \boldsymbol{u}\|_{2}\|D \boldsymbol{w}\|_{2}\right)$.

o Let us now consider the term involving the pressure

$$
\left|I_{4}\right|=\left|\left(\pi_{1}-\pi_{2}, \nabla \cdot \boldsymbol{\varphi}\right)\right| \leq\left\|\pi_{1}-\pi_{2}\right\|_{p^{\prime}}\|\nabla \cdot \varphi\|_{p} .
$$

Arguing as in the first part of the proof of Corollary 4.5 we can see that

$$
\begin{aligned}
\left\|\pi_{1}-\pi_{2}\right\|_{p^{\prime}} & \leq \tilde{\kappa}\left(\left\|\tau\left(D^{\star} \boldsymbol{u}\right)-\tau(D \boldsymbol{w})\right\|_{p^{\prime}}+\left\|\nabla^{\star} \cdot \tau\left(D^{\star} \boldsymbol{u}\right)-\nabla \cdot \tau\left(D^{\star} \boldsymbol{u}\right)\right\|_{p^{\prime}}\right) \\
& +\tilde{\kappa} \mathcal{R} e\left(\|u \otimes u-w \otimes w\|_{p^{\prime}}+\delta\left\|u_{3}^{2}\right\|_{p^{\prime}}\right)+\tilde{\kappa} \delta\left\|\sigma\left(w_{3}\right)\right\|_{p^{\prime}},
\end{aligned}
$$

where $\tilde{\kappa}$ depends only on $\Sigma, p, m$ and $n$. Taking into account (5.6) and (5.27), we have

$$
\begin{aligned}
\left\|\tau\left(D^{\star} \boldsymbol{u}\right)-\tau(D \boldsymbol{w})\right\|_{p^{\prime}} & \leq\left\|\boldsymbol{\tau}\left(D^{\star} \boldsymbol{u}\right)-\boldsymbol{\tau}(D \boldsymbol{w})\right\|_{p^{\prime}} \leq F_{4,1}\left\|D^{\star} \boldsymbol{u}-D \boldsymbol{w}\right\|_{p} \\
& \leq F_{4,1}\left(\left\|D^{\star} \boldsymbol{u}-D \boldsymbol{u}\right\|_{p}+\|D \boldsymbol{u}-D \boldsymbol{w}\|_{p}\right) \\
& \leq F_{4,2}\left(\delta+\|D \boldsymbol{u}-D \boldsymbol{w}\|_{p}\right),
\end{aligned}
$$

where $F_{4,1}=(p-1) \mathcal{F}_{1}\left(\left\|D^{\star} \boldsymbol{u}\right\|_{p}+\|D \boldsymbol{w}\|_{p}\right)$ and $F_{4,2}=F_{4,1}\left(1+m\left(\left\|u_{2}\right\|_{p}+\left\|u_{3}\right\|_{p}\right)\right)$. Moreover, by using (5.9), we obtain

$$
\left\|\nabla^{\star} \cdot \tau\left(D^{\star} \boldsymbol{u}\right)-\nabla \cdot \tau\left(D^{\star} \boldsymbol{u}\right)\right\|_{p^{\prime}} \leq F_{4,3} \delta,
$$


where $F_{4,3}=4 \sqrt{3} m \mathcal{F}_{1}\left(\left\|D^{\star} \boldsymbol{u}\right\|_{p}\right)\left\|D^{\star} \boldsymbol{u}\right\|_{p}$. Similarly, by using the Sobolev inequality (3.8) and the Korn inequality (5.1)

$$
\begin{aligned}
\|u \otimes u-w \otimes w\|_{2}+\delta\left\|u_{3}^{2}\right\|_{2} & \leq\|(u+w) \otimes(u-w)\|_{2}+\delta\left\|u_{3}\right\|_{4}^{2} \\
& \leq\|u+w\|_{4}\|u-w\|_{4}+\delta\left\|u_{3}\right\|_{4}^{2} \\
& \leq S_{2,4}\|\nabla(u-w)\|_{2}\|u+w\|_{4}+\delta\left\|u_{3}\right\|_{4}^{2} \\
& \leq S_{2,4} \sqrt{2}\|D(\boldsymbol{u}-\boldsymbol{w})\|_{2}\|u+w\|_{4}+\delta\left\|u_{3}\right\|_{4}^{2}
\end{aligned}
$$

and thus

$$
\begin{aligned}
\|u \otimes u-w \otimes w\|_{p^{\prime}}+\delta\left\|u_{3}^{2}\right\|_{p^{\prime}} & \leq|\Sigma|^{\frac{1}{p^{\prime}}-\frac{1}{2}}\left(\|u \otimes u-w \otimes w\|_{2}+\delta\left\|u_{3}^{2}\right\|_{2}\right) \\
& \leq F_{4,4}\left(\|D(\boldsymbol{u}-\boldsymbol{w})\|_{p}+\delta\right),
\end{aligned}
$$

where $F_{4,4}=|\Sigma|^{\frac{1}{p^{\prime}}-\frac{1}{2}}\left(\sqrt{2} S_{2,4}|\Sigma|^{\frac{1}{2}-\frac{1}{p}}\|u+w\|_{4}+\left\|u_{3}\right\|_{4}^{2}\right)$. On the other hand, due to (3.14) we have

$$
\left\|\sigma\left(w_{3}\right)\right\|_{p^{\prime}} \leq E_{\alpha, p}\left\|\nabla w_{3}\right\|_{2}^{\alpha}=F_{4,5}
$$

Combining these estimates, we deduce that

$$
\begin{aligned}
\left|I_{4}\right| & \leq \tilde{\kappa}\left(\left(F_{4,2}+\mathcal{R} e F_{4,4}\right)\|D \boldsymbol{u}-D \boldsymbol{w}\|_{p}+\left(F_{4,2}+F_{4,3}+\mathcal{R} e F_{4,4}+F_{4,5}\right) \delta\right)\|\nabla \cdot \boldsymbol{\varphi}\|_{p} \\
& \leq F_{4}\left(\|D(\boldsymbol{u}-\boldsymbol{w})\|_{p}+\delta\right)\|\nabla \cdot \boldsymbol{\varphi}\|_{p} .
\end{aligned}
$$

○ Finally, taking into account (3.13) we obtain

$$
\begin{aligned}
\left|I_{5}\right| & =\delta\left|\left(\sigma\left(w_{3}\right), \varphi_{2}\right)\right| \leq D_{2, \alpha} \delta\left\|\nabla w_{3}\right\|_{2}^{\alpha}\left\|\nabla \varphi_{2}\right\|_{2} \\
& \leq D_{2, \alpha} \delta\left\|\nabla w_{3}\right\|_{2}^{\alpha}\|\nabla \varphi\|_{2} \leq \sqrt{2} D_{2, \alpha} \delta\left\|\nabla w_{3}\right\|_{2}^{\alpha}\|D \varphi\|_{2} \\
& \leq F_{5} \delta\|D \varphi\|_{p},
\end{aligned}
$$

where $F_{5}=\sqrt{2} D_{2, \alpha}|\Sigma|^{\frac{1}{2}-\frac{1}{p}}\left\|\nabla w_{3}\right\|_{2}^{\alpha}$.

$\circ$ Combining (5.26), (5.28)-(15.32) yields

$$
\begin{aligned}
|(\boldsymbol{\tau}(D \boldsymbol{u})-\boldsymbol{\tau}(D \boldsymbol{w}), D \boldsymbol{\varphi})| & \leq\left(F_{1}+F_{2}+\mathcal{R} e F_{3}+F_{5}\right) \delta\|D \boldsymbol{\varphi}\|_{p}+\mathcal{R} e F_{3}\|D(\boldsymbol{u}-\boldsymbol{w})\|_{2}\|D \boldsymbol{\varphi}\|_{2} \\
& +F_{4}\left(\|D(\boldsymbol{u}-\boldsymbol{w})\|_{p}+\delta\right)\|\nabla \cdot \boldsymbol{\varphi}\|_{p} .
\end{aligned}
$$

Setting $\boldsymbol{\varphi}=\boldsymbol{u}-\boldsymbol{w}$, taking into account (5.8) and the estimates associated to $\boldsymbol{u}$ and $\boldsymbol{w}$, and using the Young inequality, it follows that for every $\varepsilon>0$,

$$
\begin{aligned}
\frac{1}{2^{p}(p-1)}\|D(\boldsymbol{u}-\boldsymbol{w})\|_{p}^{p}+\frac{1}{2}\|D(\boldsymbol{u}-\boldsymbol{w})\|_{2}^{2} & \leq(\boldsymbol{\tau}(D \boldsymbol{u})-\boldsymbol{\tau}(D \boldsymbol{w}), D(\boldsymbol{u}-\boldsymbol{w})) \\
\leq & \left(F_{1}+F_{2}+\mathcal{R} e F_{3}+F_{5}\right) \delta\|D(\boldsymbol{u}-\boldsymbol{w})\|_{p} \\
& +\mathcal{R} e F_{3}\|D(\boldsymbol{u}-\boldsymbol{w})\|_{2}^{2} \\
& +F_{4}\left(\|D(\boldsymbol{u}-\boldsymbol{w})\|_{p}+\delta\right)\left\|\frac{\delta}{B} u_{2}\right\|_{p} \\
\leq & C_{1}\left(\delta\|D(\boldsymbol{u}-\boldsymbol{w})\|_{p}+\mathcal{R} e\|D(\boldsymbol{u}-\boldsymbol{w})\|_{2}^{2}+\delta^{2}\right) \\
\leq & \varepsilon\|D(\boldsymbol{u}-\boldsymbol{w})\|_{p}^{p}+C_{2}(\varepsilon) \delta^{p^{\prime}} \\
& +C_{1}\left(\mathcal{R} e\|D(\boldsymbol{u}-\boldsymbol{w})\|_{2}^{2}+\delta^{2}\right),
\end{aligned}
$$


where $C_{1}$ is a positive constant only depending on $\Sigma, p$ and $m$. Observing that $\delta^{2}<\delta^{p^{\prime}}$, choosing $\varepsilon=\frac{1}{2^{p+1}(p-1)}$ and assuming that $C_{1} \mathcal{R} e<\frac{1}{4}$, we deduce that

$$
\frac{1}{2^{p+1}(p-1)}\|D(\boldsymbol{u}-\boldsymbol{w})\|_{p}^{p}+\frac{1}{4}\|D(\boldsymbol{u}-\boldsymbol{w})\|_{2}^{2} \leq C_{3} \delta^{p^{\prime}}
$$

and the claimed result is proved.

\section{Shear-thinning flows}

Let us now consider the case of shear-thinning fluids (corresponding to $p<2$ ). As for the shear-thickening fluids, we derive a Korn inequality, establish some estimates on the convective term and on the extra stress tensor and prove existence and uniqueness results. As previously observed in Section 4, we will restrict the exponent $p$ in order to ensure the uniqueness of the solution and carry out the approximation analysis with respect to $\delta$.

\subsection{On the Korn inequality}

Let us notice that if the classical Korn inequality can be applied to the tensor $D^{\star} \boldsymbol{u}$ with $\delta=0$ or to $D u=\left(D_{i j}^{\star} \boldsymbol{u}\right)_{i=1,2}$ with $\delta>0$, this is no more necessarily the case if we consider $D^{\star} \boldsymbol{u}$ with $\delta>0$. The difficulty, basically related with the term $D_{23}^{\star} \boldsymbol{u}=\frac{\partial u_{3}}{\partial x_{2}}-\frac{\delta}{B} u_{3}$, is overcome in the case $p=2$ by using the Hilbert setting and the fact that $u_{3 \mid \partial \Sigma}=0$. Indeed, since

$$
\left(\frac{\partial u_{3}}{\partial x_{2}}, u_{3}\right)=0
$$

we obtain

$$
\left\|D_{23}^{\star} \boldsymbol{u}\right\|_{2, B}^{2}=\left\|\frac{\partial u_{3}}{\partial x_{2}}-\frac{\delta}{B} u_{3}\right\|_{2, B}^{2}=\left\|\frac{\partial u_{3}}{\partial x_{2}}\right\|_{2, B}^{2}+\left\|\frac{\delta}{B} u_{3}\right\|_{2, B}^{2} .
$$

This argument is one of the key points in the proof of the corresponding Korn inequality (see Section 5.1) but does not apply in the $L^{p}$ setting. The issue is overcome by using the Poincaré inequality (3.11) that involves only the first component $\frac{\partial u_{3}}{\partial x_{1}}$ of the gradient $\nabla u_{3}$.

Lemma 6.1 Let $\boldsymbol{u}=\left(u_{1}, u_{2}, u_{3}\right) \in \boldsymbol{W}_{0}^{1, p}(\Sigma)$ with $1<p<\infty$. Then

$$
C_{K}\left\|\nabla^{\star} \boldsymbol{u}\right\|_{p, B} \leq\left\|D^{\star} \boldsymbol{u}\right\|_{p, B}
$$

with $C_{K}=\frac{C_{K, 1}(n m)^{-\frac{1}{p}}}{2(1+\delta m)}$, where $C_{K, 1}$ is the classical Korn constant in $\boldsymbol{W}_{0}^{1, p}(\Sigma)$.

Proof. Let us first observe that due to (3.11), we have

$$
\left\|\frac{\delta}{B} u_{3}\right\|_{p, B} \leq\left\|\frac{\delta}{B} \frac{\partial u_{3}}{\partial x_{1}}\right\|_{p, B} \leq 2 \delta m\left\|D_{13}^{\star} \boldsymbol{u}\right\|_{p, B} .
$$

It follows that

$$
\begin{aligned}
\left\|D_{23} \boldsymbol{u}\right\|_{p, B}^{p} & \leq\left(\left\|D_{23}^{\star} \boldsymbol{u}\right\|_{p, B}+\frac{1}{2}\left\|\frac{\delta}{B} u_{3}\right\|_{p, B}\right)^{p} \\
& \leq\left(\left\|D_{23}^{\star} \boldsymbol{u}\right\|_{p, B}+\delta m\left\|D_{13}^{\star} \boldsymbol{u}\right\|_{p, B}\right)^{p} \\
& \leq 2^{p-1}\left(\left\|D_{23}^{\star} \boldsymbol{u}\right\|_{p, B}^{p}+(\delta m)^{p}\left\|D_{13}^{\star} \boldsymbol{u}\right\|_{p, B}^{p}\right)
\end{aligned}
$$


and thus

$$
\begin{aligned}
\|D \boldsymbol{u}\|_{p, B}^{p} & =\|D u\|_{p, B}^{p}+2\left\|D_{13} \boldsymbol{u}\right\|_{p, B}^{p}+2\left\|D_{23} \boldsymbol{u}\right\|_{p, B}^{p} \\
& \leq\|D u\|_{p, B}^{p}+2\left\|D_{13}^{\star} \boldsymbol{u}\right\|_{p, B}^{p}+2^{p}\left\|D_{23}^{\star} \boldsymbol{u}\right\|_{p, B}^{p}+(2 \delta m)^{p}\left\|D_{13}^{\star} \boldsymbol{u}\right\|_{p, B}^{p} .
\end{aligned}
$$

On the other hand, taking into account the definition of $\nabla^{\star}$ and using the classical Korn inequality, we can easily see that

$$
\begin{aligned}
\left\|\nabla^{\star} \boldsymbol{u}\right\|_{p, B}^{p} & =\|\nabla \boldsymbol{u}\|_{p, B}^{p}+\left\|\frac{\delta}{B} u_{2}\right\|_{p, B}^{p}+\left\|\frac{\delta}{B} u_{3}\right\|_{p, B}^{p} \\
& \leq n\|\nabla \boldsymbol{u}\|_{p}^{p}+\left\|\frac{\delta}{B} u_{2}\right\|_{p, B}^{p}+\left\|\frac{\delta}{B} u_{3}\right\|_{p, B}^{p} \\
& \leq \frac{n}{C_{K, 1}^{p}}\|D \boldsymbol{u}\|_{p}^{p}+\left\|\frac{\delta}{B} u_{2}\right\|_{p, B}^{p}+\left\|\frac{\delta}{B} u_{3}\right\|_{p, B}^{p} \\
& \leq \frac{m n}{C_{K, 1}^{p}}\|D \boldsymbol{u}\|_{p, B}^{p}+\left\|\frac{\delta}{B} u_{2}\right\|_{p, B}^{p}+\left\|\frac{\delta}{B} u_{3}\right\|_{p, B}^{p} .
\end{aligned}
$$

Combining (6.2)-6.4), we deduce that

$$
\begin{aligned}
& \left\|\nabla^{\star} \boldsymbol{u}\right\|_{p, B}^{p} \\
& \leq \frac{m n}{C_{K, 1}^{p}}\left(\|D u\|_{p, B}^{p}+2\left\|D_{13}^{\star} \boldsymbol{u}\right\|_{p, B}^{p}+2^{p}\left\|D_{23}^{\star} \boldsymbol{u}\right\|_{p, B}^{p}+(2 \delta m)^{p}\left\|D_{13}^{\star} \boldsymbol{u}\right\|_{p, B}^{p}\right) \\
& +(2 \delta m)^{p}\left\|D_{13}^{\star} \boldsymbol{u}\right\|_{p, B}^{p}+\left\|D_{33}^{\star} \boldsymbol{u}\right\|_{p, B}^{p} \\
& \leq \frac{m n}{C_{K, 1}^{p}}\left(\|D u\|_{p, B}^{p}+\left(2+(2 \delta m)^{p}\left(1+\frac{C_{K, 1}^{p}}{m n}\right)\right)\left\|D_{13}^{\star} \boldsymbol{u}\right\|_{p, B}^{p}+2^{p}\left\|D_{23}^{\star} \boldsymbol{u}\right\|_{p, B}^{p}+\left\|D_{33}^{\star} \boldsymbol{u}\right\|_{p, B}^{p}\right) \\
& \leq \frac{(2+2 \delta m)^{p} m n}{C_{K, 1}^{p}}\left(\|D u\|_{p, B}^{p}+2\left\|D_{13}^{\star} \boldsymbol{u}\right\|_{p, B}^{p}+2\left\|D_{23}^{\star} \boldsymbol{u}\right\|_{p, B}^{p}+\left\|D_{33}^{\star} \boldsymbol{u}\right\|_{p, B}^{p}\right) \\
& =\frac{1}{C_{K}^{p}}\left\|D^{\star} \boldsymbol{u}\right\|_{p, B}^{p}
\end{aligned}
$$

and the claimed result is proved.

\subsection{Estimates on the convective term and extra stress tensor}

We begin by a continuity property in $\boldsymbol{W}_{0}^{1, p}(\Sigma)$ of the trilinear form $a_{\star}$.

Lemma 6.2 Let $\boldsymbol{u}, \boldsymbol{v}$ and $\boldsymbol{w}$ be in $\boldsymbol{W}_{0}^{1, p}(\Sigma)$ with $\frac{3}{2} \leq p<2$. Then the following estimate holds

$$
\left|a_{\star}(\boldsymbol{u}, \boldsymbol{v}, B \boldsymbol{w})\right| \leq \kappa_{6}\left\|D^{\star} \boldsymbol{u}\right\|_{p, B}\left\|D^{\star} \boldsymbol{v}\right\|_{p, B}\left\|D^{\star} \boldsymbol{w}\right\|_{p, B}
$$

with $\kappa_{6}=\frac{n m^{\frac{3}{p}}}{C_{K}^{3}}\left(S_{p, 2 p^{\prime}}\right)^{2}$ and where $C_{K}$ is the Korn constant given in (6.1).

Proof. Hölder's inequality and Sobolev's inequality with $r=2 p^{\prime}$ and $q=p$ show that

$$
\begin{aligned}
\left|a_{\star}(\boldsymbol{u}, \boldsymbol{v}, B \boldsymbol{w})\right| & \leq n\|\boldsymbol{u}\|_{2 p^{\prime}}\left\|\nabla^{\star} \boldsymbol{v}\right\|_{p}\|\boldsymbol{w}\|_{2 p^{\prime}} \\
& \leq n\left(S_{p, 2 p^{\prime}}\right)^{2}\|\nabla \boldsymbol{u}\|_{p}\left\|\nabla^{\star} \boldsymbol{v}\right\|_{p}\|\nabla \boldsymbol{w}\|_{p} \\
& \leq n m^{\frac{3}{p}}\left(S_{p, 2 p^{\prime}}\right)^{2}\|\nabla \boldsymbol{u}\|_{p, B}\left\|\nabla^{\star} \boldsymbol{v}\right\|_{p, B}\|\nabla \boldsymbol{w}\|_{p, B} .
\end{aligned}
$$

The estimate follows by using the Korn inequality (6.1).

In the remaining part of this section, we study some properties of the extra stress tensor and derive some associated estimates. We begin by an auxiliary result that will be useful in the sequel. 
Lemma 6.3 Let $1<p<2$ and let $H_{1} \in L^{\frac{p}{2-p}}(\Sigma), H_{2} \in L^{1}(\Sigma)$ and $H_{3} \in L^{p}(\Sigma)$ be non negative functions satisfying

$$
H_{3}(x)^{2} \leq H_{1}(x) H_{2}(x) \quad \text { for a.e. } x \in \Sigma \text {. }
$$

Then,

$$
\left\|H_{3}\right\|_{p}^{2} \leq\left\|H_{1}\right\|_{\frac{p}{2-p}}\left\|H_{2}\right\|_{1} .
$$

Proof. Taking into account the condition satisfied by $H_{1}, H_{2}, H_{3}$, integrating and using the Hölder inequality, we obtain

$$
\begin{aligned}
\left\|H_{3}\right\|_{p}^{p} & =\int_{\Sigma}\left(H_{3}(x)^{2}\right)^{\frac{p}{2}} d x \leq \int_{\Sigma} H_{1}(x)^{\frac{p}{2}} H_{2}(x)^{\frac{p}{2}} d x \\
& \leq\left\|H_{1}^{\frac{p}{2}}\right\|_{\frac{2}{2-p}}\left\|H_{2}^{\frac{p}{2}}\right\|_{\frac{2}{p}}=\left\|H_{1}\right\|_{\frac{p}{2-p}}^{\frac{p}{2}}\left\|H_{2}\right\|_{1}^{\frac{p}{2}}
\end{aligned}
$$

and the proof is complete.

The next result deals with continuity, coercivity and monotonicity results for the extra stress tensor $\boldsymbol{\tau}$.

Proposition 6.4 Assume that $1<p<2$ and let $\boldsymbol{f}, \boldsymbol{g} \in \boldsymbol{L}^{p}\left(\Sigma, \mathbb{R}^{3 \times 3}\right)$. Then the following estimates hold

Continuity.

$$
\begin{gathered}
\left\|\left(1+|\boldsymbol{f}|^{2}\right)^{\frac{p-2}{2}} \boldsymbol{g}\right\|_{p^{\prime}, B} \leq\|\boldsymbol{g}\|_{p, B}^{p-1} \quad \text { if }|\boldsymbol{g}| \leq|\boldsymbol{f}|, \\
\|\boldsymbol{\tau}(\boldsymbol{f})-\boldsymbol{\tau}(\boldsymbol{g})\|_{p^{\prime}, B} \leq 2 C_{p}\|\boldsymbol{f}-\boldsymbol{g}\|_{p, B}^{p-1} \quad \text { with } C_{p}=1+2^{\frac{2-p}{2}},
\end{gathered}
$$

Coercivity.

$$
(\boldsymbol{\tau}(\boldsymbol{f}), B \boldsymbol{f}) \geq \frac{2\|\boldsymbol{f}\|_{p, B}^{2}}{\left(\|B\|_{1}+\|\boldsymbol{f}\|_{p, B}^{p}\right)^{\frac{2-p}{p}}},
$$

Monotonicity.

$$
(\boldsymbol{\tau}(\boldsymbol{f})-\boldsymbol{\tau}(\boldsymbol{g}), B(\boldsymbol{f}-\boldsymbol{g})) \geq \frac{2(p-1)\|\boldsymbol{f}-\boldsymbol{g}\|_{p, B}^{2}}{\left(\|B\|_{1}+\|\boldsymbol{f}\|_{p, B}^{p}+\|\boldsymbol{g}\|_{p, B}^{p}\right)^{\frac{2-p}{p}}} .
$$

Proof. Standard calculation show that if $|\boldsymbol{g}| \leq|\boldsymbol{f}|$, then

$$
\begin{aligned}
\left\|\left(1+|\boldsymbol{f}|^{2}\right)^{\frac{p-2}{2}} \boldsymbol{g}\right\|_{p^{\prime}, B}^{p^{\prime}} & =\int_{\Sigma} B\left(\left(1+|\boldsymbol{f}|^{2}\right)^{\frac{p-2}{2}}|\boldsymbol{g}|\right)^{p^{\prime}} d x \\
& \leq \int_{\Sigma} B\left(\left(1+|\boldsymbol{g}|^{2}\right)^{\frac{p-2}{2}}|\boldsymbol{g}|\right)^{p^{\prime}} d x \\
& \leq \int_{\Sigma} B\left(\left(|\boldsymbol{g}|^{2}\right)^{\frac{p-2}{2}}|\boldsymbol{g}|\right)^{p^{\prime}} d x=\|\boldsymbol{g}\|_{p, B}^{p}
\end{aligned}
$$

which gives (6.5). On the other hand, due to the monotonicity property in Lemma 3.2, we have

$$
\begin{aligned}
\frac{1}{2(p-1)}(\boldsymbol{\tau}(f)-\boldsymbol{\tau}(\boldsymbol{g})): B(\boldsymbol{f}-\boldsymbol{g}) & \geq B\left(1+|\boldsymbol{f}|^{2}+|\boldsymbol{g}|^{2}\right)^{\frac{2-p}{2}}|\boldsymbol{f}-\boldsymbol{g}|^{2} \\
& =\left(B^{\frac{2}{p}}+\left|B^{\frac{1}{p}} \boldsymbol{f}\right|^{2}+\left|B^{\frac{1}{p}} \boldsymbol{g}\right|^{2}\right)^{\frac{2-p}{2}}\left|B^{\frac{1}{p}}(\boldsymbol{f}-\boldsymbol{g})\right|^{2} .
\end{aligned}
$$


Set

$$
\begin{aligned}
& H_{1}=\left(B^{\frac{2}{p}}+\left|B^{\frac{1}{p}} \boldsymbol{f}\right|^{2}+\left|B^{\frac{1}{p}} \boldsymbol{g}\right|^{2}\right)^{\frac{2-p}{2}}, \\
& H_{2}=\frac{1}{2(p-1)}(\boldsymbol{\tau}(\boldsymbol{f})-\boldsymbol{\tau}(\boldsymbol{g}): B(\boldsymbol{f}-\boldsymbol{g})), \quad H_{3}=\left|B^{\frac{1}{p}}(\boldsymbol{f}-\boldsymbol{g})\right| .
\end{aligned}
$$

Since $\boldsymbol{f}$ and $\boldsymbol{g}$ belong to $\boldsymbol{L}^{p}\left(\Sigma, \mathbb{R}^{3 \times 3}\right)$, it is easy to see that $H_{1} \in L^{\frac{p}{2-p}}(\Sigma), H_{2} \in L^{1}(\Sigma)$ and $H_{3} \in L^{p}(\Sigma)$ and that due to (6.9), we have

$$
H_{3}(x)^{2} \leq H_{1}(x) H_{2}(x) \quad \text { for a.e. } x \in \Sigma .
$$

Due to Lemma 6.3, we obtain

$$
\begin{aligned}
2(p-1)\|\boldsymbol{f}-\boldsymbol{g}\|_{p, B}^{2} & \leq\left\|\left(B^{\frac{2}{p}}+\left|B^{\frac{1}{p}} \boldsymbol{f}\right|^{2}+\left|B^{\frac{1}{p}} \boldsymbol{g}\right|^{2}\right)^{\frac{2-p}{2}}\right\|\left\|_{\frac{p}{2-p}}\right\|(\boldsymbol{\tau}(\boldsymbol{f})-\boldsymbol{\tau}(\boldsymbol{g})): B(\boldsymbol{f}-\boldsymbol{g}) \|_{1} \\
& \leq\left(\|B\|_{1}+\|\boldsymbol{f}\|_{p, B}^{p}+\|\boldsymbol{g}\|_{p, B}^{p}\right)^{\frac{2-p}{p}}(\boldsymbol{\tau}(\boldsymbol{f})-\boldsymbol{\tau}(\boldsymbol{g}), B(\boldsymbol{f}-\boldsymbol{g}))
\end{aligned}
$$

which gives (6.8). Estimate (6.7) can be obtained very similarly by using the coercivity condition in Lemma 3.2. Finally, by taking into account Lemma 3.2, we have

$$
\|\boldsymbol{\tau}(\boldsymbol{f})-\boldsymbol{\tau}(\boldsymbol{g})\|_{p^{\prime}, B} \leq 2 C_{p}\left\||\boldsymbol{f}-\boldsymbol{g}|^{p-1}\right\|_{p^{\prime}, B}=2 C_{p}\|\boldsymbol{f}-\boldsymbol{g}\|_{p, B}^{p-1}
$$

which gives estimate (6.6) .

Proposition 6.5 Assume that $\frac{3}{2} \leq p<2$ and let $\boldsymbol{f} \in \boldsymbol{L}^{p}\left(\Sigma, \mathbb{R}^{3 \times 3}\right)$. Then the following estimate holds

$$
\left\|\nabla^{\star} \cdot \boldsymbol{\tau}(\boldsymbol{f})-\nabla \cdot \boldsymbol{\tau}(\boldsymbol{f})\right\|_{p^{\prime}} \leq 4 \delta m\left(\|f\|_{p}^{p-1}+\left\|f_{33}\right\|_{p}^{p-1}+\left\|f_{23}\right\|_{p}^{p-1}\right) .
$$

where $f=\left(f_{i j}\right)_{i j=1,2}$.

Proof. Taking into account (6.5), the estimate can be derived by following step by step the proof of Proposition 5.6.

\subsection{Existence and uniqueness of shear-thinning flows}

Proposition 6.6 Assume that $\frac{3}{2} \leq p<2$ and let $\boldsymbol{u}$ be a weak solution of (4.1). Then, estimates (4.10)-(4.13) hold.

Proof. The proof is split into three steps.

Step 1. Let us set $\boldsymbol{\varphi}=\boldsymbol{u}$ in the weak formulation (4.1) and use Lemma 5.3 and (6.7) to obtain

$$
\frac{2\left\|D^{\star} \boldsymbol{u}\right\|_{p, B}^{2}}{\left(\|B\|_{1}+\left\|D^{\star} \boldsymbol{u}\right\|_{p, B}^{p}\right)^{\frac{2-p}{p}}} \leq\left(\boldsymbol{\tau}\left(D^{\star} \boldsymbol{u}\right), B D^{\star} \boldsymbol{u}\right)=\left(G, u_{3}\right) .
$$

On the other hand, classical arguments together with Poincaré inequality (3.9) give

$$
\left(G, u_{3}\right) \leq 2 \kappa_{1}\left\|u_{3}\right\|_{p, B} \leq 2 \kappa_{1}\left\|\frac{\partial u_{3}}{\partial x_{1}}\right\|_{p, B},
$$

where $\kappa_{1}=\frac{m^{\frac{1}{p}}}{2}|G||\Sigma|^{\frac{1}{p^{\prime}}}$. Observing that

$$
\left\|D^{\star} \boldsymbol{u}\right\|_{p, B}^{p}=\int_{\Sigma} B\left(\left|D^{\star} \boldsymbol{u}\right|^{2}\right)^{\frac{p}{2}} d x \geq\left\|\frac{\partial u_{3}}{\partial x_{1}}\right\|_{p, B}^{p},
$$


we deduce that

$$
\left(G, u_{3}\right) \leq 2 \kappa_{1}\left\|D^{\star} \boldsymbol{u}\right\|_{p, B}
$$

Due to (6.11) and (6.12), we have

$$
\left\|D^{\star} \boldsymbol{u}\right\|_{p, B} \leq \kappa_{1}\left(\|B\|_{1}+\left\|D^{\star} \boldsymbol{u}\right\|_{p, B}^{p}\right)^{\frac{2-p}{p}}
$$

and thus

$$
\left\|D^{\star} \boldsymbol{u}\right\|_{p, B}^{\frac{p}{2-p}} \leq \kappa_{1}^{\frac{p}{2-p}}\left(\|B\|_{1}+\left\|D^{\star} \boldsymbol{u}\right\|_{p, B}^{p}\right) .
$$

The Young inequality yields

$$
\kappa_{1}^{\frac{p}{2-p}}\left\|D^{\star} \boldsymbol{u}\right\|_{p, B}^{p} \leq(2-p)\left\|D^{\star} \boldsymbol{u}\right\|_{p, B}^{\frac{p}{2-p}}+(p-1) \kappa_{1}^{\frac{p}{(2-p)(p-1)}}
$$

and by combining (6.13) and (6.14), we deduce that

$$
(p-1)\left\|D^{\star} \boldsymbol{u}\right\|_{p, B}^{\frac{p}{2-p}} \leq \kappa_{1}^{\frac{p}{2-p}}\|B\|_{1}+(p-1) \kappa_{1}^{\frac{p}{(2-p)(p-1)}} .
$$

Consequently

$$
\left\|D^{\star} \boldsymbol{u}\right\|_{p, B} \leq \kappa_{1}\left(\frac{\|B\|_{1}}{p-1}+\kappa_{1}^{p^{\prime}}\right)^{\frac{2-p}{p}}
$$

and estimate (4.10) is proved.

Step 2. Let us now prove (4.11). Similar arguments together with the coercivity property and (6.12) show that

$$
\begin{aligned}
\left\|D^{\star} \boldsymbol{u}\right\|_{p, B}^{p} & =\int_{\Sigma_{u}} B\left|D^{\star} \boldsymbol{u}\right|^{p} d x+\int_{\Sigma \backslash \Sigma_{\boldsymbol{u}}} B\left|D^{\star} \boldsymbol{u}\right|^{p} d x \\
& \leq \int_{\Sigma_{\boldsymbol{u}}} \frac{\left|B^{\frac{1}{p}} D^{\star} \boldsymbol{u}\right|^{2}}{\left|B^{\frac{1}{p}} D^{\star} \boldsymbol{u}\right|^{2-p}} d x+\|B\|_{1} \\
& \leq 2^{\frac{2-p}{2}} \int_{\Sigma_{\boldsymbol{u}}} \frac{\left|B^{\frac{1}{p}} D^{\star} \boldsymbol{u}\right|^{2}}{\left(B^{\frac{2}{p}}+\left|B^{\frac{1}{p}} D^{\star} \boldsymbol{u}\right|^{2}\right)^{\frac{2-p}{2}}} d x+\|B\|_{1} \\
& \leq 2^{\frac{2-p}{2}} \int_{\Sigma_{\boldsymbol{u}}} \boldsymbol{\tau}\left(D^{\star} \boldsymbol{u}\right): B D^{\star} \boldsymbol{u} d x+\|B\|_{1} \\
& \leq 2^{\frac{2-p}{2}}\left(\boldsymbol{\tau}\left(D^{\star} \boldsymbol{u}\right), B D^{\star} \boldsymbol{u}\right)+\|B\|_{1}=2^{\frac{2-p}{2}}\left(G, u_{3}\right)+\|B\|_{1} \\
& \leq 2^{\frac{2-p}{2}} \kappa_{1}\left\|D^{\star} \boldsymbol{u}\right\|_{p, B}+\|B\|_{1},
\end{aligned}
$$

where $\Sigma_{\boldsymbol{u}}=\left\{x \in \Sigma\left|B^{\frac{1}{p}}\right| D^{\star} \boldsymbol{u}(x) \mid \geq 1\right\}$. The Young inequality yields

$$
2^{\frac{2-p}{2}} \kappa_{1}\left\|D^{\star} \boldsymbol{u}\right\|_{p, B} \leq \frac{1}{p^{\prime}}\left(2^{\frac{2-p}{2}} \kappa_{1}\right)^{p^{\prime}}+\frac{1}{p}\left\|D^{\star} \boldsymbol{u}\right\|_{p, B}^{p}
$$

and the claimed result follows by combining (6.15) and (6.16).

Step 3. By taking into account (6.2), we have

$$
\begin{aligned}
\left\|\nabla u_{3}\right\|_{p, B} & \leq\left\|\frac{\partial u_{3}}{\partial x_{1}}\right\|_{p, B}+\left\|\frac{\partial u_{3}}{\partial x_{2}}-\frac{\delta}{B} u_{3}\right\|_{p, B}+\left\|\frac{\delta}{B} u_{3}\right\|_{p, B} \\
& \leq 2(1+\delta m)\left\|D_{13}^{\star} \boldsymbol{u}\right\|_{p, B}+2\left\|D_{23}^{\star} \boldsymbol{u}\right\|_{p, B} \\
& \leq 2^{2-p}(1+\delta m)\left\|D^{\star} \boldsymbol{u}\right\|_{p, B}
\end{aligned}
$$


and estimate (4.12) is then a consequence of (4.10). Let us finally prove (4.13). Setting $\varphi=u$ in (4.1) we obtain

$$
\int_{\Sigma}\left(B^{\frac{2}{p}}+\left|B^{\frac{1}{p}} D^{\star} \boldsymbol{u}\right|^{2}\right)^{\frac{p-2}{2}}\left|B^{\frac{1}{p}} D u\right|^{2} d x \leq \frac{1}{2} \mathcal{R} e \delta\left(u_{3}^{2}, u_{2}\right) .
$$

Unlike the proof of estimates (4.10) and (4.11), the coercivity property is not immediat and cannot be used. Let us then set

$$
\begin{aligned}
& H_{1}=\left(B^{\frac{2}{p}}+\left|B^{\frac{1}{p}} D^{\star} \boldsymbol{u}\right|^{2}\right)^{\frac{2-p}{2}}, \\
& H_{2}=\left(B^{\frac{2}{p}}+\left|B^{\frac{1}{p}} D^{\star} \boldsymbol{u}\right|^{2}\right)^{\frac{p-2}{2}}\left|B^{\frac{1}{p}} D u\right|^{2}, \quad H_{3}=\left|B^{\frac{1}{p}} D u\right| .
\end{aligned}
$$

It is easy to see that $H_{1} \in \boldsymbol{L}^{\frac{p}{2-p}}(\Sigma)$ and that $H_{3} \in \boldsymbol{L}^{p}(\Sigma)$. Moreover, since $\left|D^{\star} \boldsymbol{u}\right| \geq|D u|$, we have

$$
H_{2} \leq\left(B^{\frac{2}{p}}+\left|B^{\frac{1}{p}} D u\right|^{2}\right)^{\frac{p-2}{2}}\left|B^{\frac{1}{p}} D u\right|^{2} \leq\left|B^{\frac{1}{p}} D u\right|^{p} \in L^{1}(\Sigma) .
$$

Using Lemma 6.3, we deduce that

$$
\begin{aligned}
\|D u\|_{p, B}^{2} & \leq\left\|\left(B^{\frac{2}{p}}+\left|B^{\frac{1}{p}} D^{\star} \boldsymbol{u}\right|^{2}\right)^{\frac{2-p}{2}}\right\|_{\frac{p}{2-p}}\left\|\left(B^{\frac{2}{p}}+\left|B^{\frac{1}{p}} D^{\star} \boldsymbol{u}\right|^{2}\right)^{\frac{p-2}{2}}\left|B^{\frac{1}{p}} D u\right|^{2}\right\|_{1} \\
& \leq\left(\|B\|_{1}+\left\|D^{\star} \boldsymbol{u}\right\|_{p, B}^{p}\right)^{\frac{2-p}{p}}\left\|\left(B^{\frac{2}{p}}+\left|B^{\frac{1}{p}} D^{\star} \boldsymbol{u}\right|^{2}\right)^{\frac{p-2}{2}}\left|B^{\frac{1}{p}} D u\right|^{2}\right\|_{1} .
\end{aligned}
$$

On the other hand, taking into account (3.15) with $\alpha=2$ and $q=p$ and the classical Korn inequality, we have

$$
\begin{aligned}
\left|\left(u_{3}^{2}, u_{2}\right)\right| & \leq\left(S_{p, 2 p^{\prime}}\right)^{3}\left\|\nabla u_{3}\right\|_{p}^{2}\left\|\nabla u_{2}\right\|_{p} \leq \frac{\left(S_{p, 2 p^{\prime}}\right)^{3}}{C_{K, 1}}\left\|\nabla u_{3}\right\|_{p}^{2}\|D u\|_{p} \\
& \leq \tilde{\kappa}\left\|\nabla u_{3}\right\|_{p, B}^{2}\|D u\|_{p, B},
\end{aligned}
$$

where $\tilde{\kappa}=\frac{1}{C_{K, 1}}\left(m^{\frac{1}{p}} S_{p, 2 p^{\prime}}\right)^{3}$. Combining (6.17)-(16.19) and taking into account (4.11), we get

$$
\begin{aligned}
\|D u\|_{p, B} & \leq \frac{\tilde{\kappa}}{2}\left\|\nabla u_{3}\right\|_{p, B}^{2}\left(\|B\|_{1}+\left\|D^{\star} \boldsymbol{u}\right\|_{p, B}^{p}\right)^{\frac{2-p}{p}} \delta \mathcal{R} e \\
& \leq \frac{\tilde{\kappa}}{2}\left\|\nabla u_{3}\right\|_{p, B}^{2}\left(\frac{2 p-1}{p-1}\|B\|_{1}+\left(2^{\frac{2-p}{2}} \kappa_{1}\right)^{p^{\prime}}\right)^{\frac{2-p}{p}} \delta \mathcal{R} e \\
& \leq \tilde{\kappa}\left\|\nabla u_{3}\right\|_{p, B}^{2}\left(\frac{\|B\|_{1}}{p-1}+\kappa_{1}^{p^{\prime}}\right)^{\frac{2-p}{p}} \delta \mathcal{R} e
\end{aligned}
$$

and the conclusion follows from estimate (4.12).

Proof of Theorem 4.3. Let $\boldsymbol{u}^{k}$ be a standard Galerkin approximation. Arguments similar to those used in the proof of Proposition [6.6 show that

$$
\left\|D^{\star} \boldsymbol{u}^{k}\right\|_{p, B}^{p} \leq p^{\prime}\|B\|_{1}+\left(2^{\frac{2-p}{2}} \kappa_{1}\right)^{p^{\prime}}
$$


and the sequence $\left(D^{\star} \boldsymbol{u}^{k}\right)_{k}$ is then bounded in $\boldsymbol{L}^{p}(\Sigma)$. Taking into account the Korn inequality (6.1), we deduce that $\left(\nabla^{\star} \boldsymbol{u}^{k}\right)_{k}$ is bounded in $\boldsymbol{L}^{p}(\Sigma)$ and thus $\left(\nabla \boldsymbol{u}^{k}\right)_{k}$ is bounded in $\boldsymbol{L}^{p}(\Sigma)$. Moreover, the continuity property (6.5) implies that

$$
\left\|\boldsymbol{\tau}\left(D^{\star} \boldsymbol{u}^{k}\right)\right\|_{p^{\prime}, B} \leq 2\left\|D^{\star} \boldsymbol{u}^{k}\right\|_{p, B}^{p-1}
$$

and the sequence $\left(B^{\frac{1}{p^{\prime}}} \boldsymbol{\tau}\left(D^{\star} \boldsymbol{u}^{k}\right)\right)_{k}$ is bounded in $\boldsymbol{L}^{p^{\prime}}(\Sigma)$. There then exist a subsequence, still indexed by $k, \boldsymbol{u} \in \boldsymbol{V}_{B}^{p}$ and $\widetilde{\boldsymbol{\tau}} \in \boldsymbol{L}^{p^{\prime}}(\Sigma)$ such that $\left(\nabla \boldsymbol{u}^{k}\right)_{k}$ converges to $\boldsymbol{u}$ weakly in $\boldsymbol{L}^{p}(\Sigma)$ and $\left(B^{\frac{1}{p^{\prime}} \boldsymbol{\tau}}\left(D^{\star} \boldsymbol{u}^{k}\right)\right)_{k}$ converges to $\widetilde{\boldsymbol{\tau}}$ weakly in $\boldsymbol{L}^{p^{\prime}}(\Sigma)$. Moreover, since $p>\frac{4}{3}$, by using compactness results on Sobolev spaces, we deduce that $\left(\boldsymbol{u}^{k}\right)_{k}$ strongly converges to $\boldsymbol{u}$ in $L^{p^{\prime}}(\Sigma)$. Taking into account these convergence results, we deduce that for every $\varphi \in \mathcal{V}_{B}$, we have

$$
\begin{aligned}
\left|a_{\star}\left(\boldsymbol{u}^{k}, \boldsymbol{u}^{k}, B \boldsymbol{\varphi}\right)-a_{\star}(\boldsymbol{u}, \boldsymbol{u}, B \boldsymbol{\varphi})\right| & \leq\left|a\left(\boldsymbol{u}^{k}-\boldsymbol{u}, \boldsymbol{u}^{k}, B \boldsymbol{\varphi}\right)\right|+\left|a_{\star}\left(\boldsymbol{u}, \boldsymbol{u}^{k}-\boldsymbol{u}, B \boldsymbol{\varphi}\right)\right| \\
& =\left|a_{\star}\left(\boldsymbol{u}^{k}-\boldsymbol{u}, \boldsymbol{u}^{k}, B \boldsymbol{\varphi}\right)\right|+\left|a_{\star}\left(B \boldsymbol{u}, \boldsymbol{\varphi}, \boldsymbol{u}^{k}-\boldsymbol{u}\right)\right| \\
& \leq\left(\left\|\nabla^{\star} \boldsymbol{u}^{k}\right\|_{p, B}\|\boldsymbol{\varphi}\|_{\infty}+\|\boldsymbol{u}\|_{p, B}\|\nabla \boldsymbol{\varphi}\|_{\infty}\right)\left\|\boldsymbol{u}^{k}-\boldsymbol{u}\right\|_{p^{\prime}, B} \\
& \longrightarrow 0 \quad \text { when } k \rightarrow+\infty .
\end{aligned}
$$

Moreover, by passing to the limit in

$$
\left(\boldsymbol{\tau}\left(D^{\star} \boldsymbol{u}^{k}\right), B D^{\star} \boldsymbol{\varphi}\right)+\mathcal{R} e a_{\star}\left(\boldsymbol{u}^{k}, \boldsymbol{u}^{k}, B \boldsymbol{\varphi}\right)=\left(G, \varphi_{3}\right) \quad \text { for all } \boldsymbol{\varphi} \in \mathcal{V}_{B},
$$

we obtain

$$
\left(\widetilde{\boldsymbol{\tau}}, B^{\frac{1}{p}} D^{\star} \boldsymbol{\varphi}\right)+\mathcal{R} e a_{\star}(\boldsymbol{u}, \boldsymbol{u}, B \boldsymbol{\varphi})=\left(G, \varphi_{3}\right) \quad \text { for all } \boldsymbol{\varphi} \in \mathcal{V}_{B}
$$

and by using the fact that $\mathcal{V}_{B}$ is dense in $\boldsymbol{V}_{B}^{p}$, we deduce that

$$
\left(\widetilde{\boldsymbol{\tau}}, B^{\frac{1}{p}} D^{\star} \boldsymbol{\varphi}\right)+\mathcal{R} e a_{\star}(\boldsymbol{u}, \boldsymbol{u}, B \boldsymbol{\varphi})=\left(G, \varphi_{3}\right) \quad \text { for all } \boldsymbol{\varphi} \in \boldsymbol{V}_{B}^{p} .
$$

The rest of the proof for the existence of a weak solution is very similar to the first step in the proof of Theorem 4.2 and is omitted. To prove the uniqueness result, let us assume that $\boldsymbol{u}$ and $\boldsymbol{v}$ are two weak solutions of (2.7). Setting $\boldsymbol{\varphi}=\boldsymbol{u}-\boldsymbol{v}$ in the corresponding weak formulation and taking into account Lemma 5.3 and (6.8), we obtain

$$
\begin{aligned}
\frac{\left\|D^{\star}(\boldsymbol{u}-\boldsymbol{v})\right\|_{p, B}^{2}}{\left(\|B\|_{1}+\left\|D^{\star} \boldsymbol{u}\right\|_{p, B}^{p}+\left\|D^{\star} \boldsymbol{v}\right\|_{p, B}^{p}\right)^{\frac{2-p}{p}}} & \leq\left(\boldsymbol{\tau}\left(D^{\star} \boldsymbol{u}\right)-\boldsymbol{\tau}\left(D^{\star} \boldsymbol{v}\right), B D^{\star}(\boldsymbol{u}-\boldsymbol{v})\right) \\
& =\mathcal{R} e a_{\star}(\boldsymbol{v}, \boldsymbol{v}, B(\boldsymbol{u}-\boldsymbol{v}))-\mathcal{R} e a_{\star}(\boldsymbol{u}, \boldsymbol{u}, B(\boldsymbol{u}-\boldsymbol{v})) \\
& =-\mathcal{R e} a(\boldsymbol{u}-\boldsymbol{v}, \boldsymbol{v}, B(\boldsymbol{u}-\boldsymbol{v})) .
\end{aligned}
$$

Lemma 6.2 and estimate (4.10) then yield

$$
\begin{aligned}
\left|a_{\star}(\boldsymbol{u}-\boldsymbol{v}, \boldsymbol{v}, \boldsymbol{u}-\boldsymbol{v})\right| & \leq \kappa_{6}\left\|D^{\star}(\boldsymbol{u}-\boldsymbol{v})\right\|_{p, B}^{2}\left\|D^{\star} \boldsymbol{v}\right\|_{p, B} \\
& \leq \kappa_{1} \kappa_{6}\left(\frac{\|B\|_{1}}{p-1}+\kappa_{1}^{p^{\prime}}\right)^{\frac{2-p}{p}}\left\|D^{\star}(\boldsymbol{u}-\boldsymbol{v})\right\|_{p, B}^{2} .
\end{aligned}
$$

On the other hand, by taking into account estimate (4.11), we have

$$
\begin{aligned}
\left(\|B\|_{1}+\left\|D^{\star} \boldsymbol{u}\right\|_{p, B}^{p}+\left\|D^{\star} \boldsymbol{v}\right\|_{p, B}^{p}\right)^{\frac{2-p}{p}} & \leq\left(\frac{3 p-1}{p-1}\|B\|_{1}+\left(2^{\frac{2-p}{2}} \kappa_{1}\right)^{p^{\prime}}\right)^{\frac{2-p}{p}} \\
& \leq 2\left(\frac{\|B\|_{1}}{p-1}+\kappa_{1}^{p^{\prime}}\right)^{\frac{2-p}{p}} .
\end{aligned}
$$


By combining (6.20), 6.21) and (6.22), we deduce that

$$
\left(\frac{1}{2\left(\frac{\|B\|_{1}}{p-1}+\kappa_{1}^{p^{\prime}}\right)^{\frac{2-p}{p}}}-\mathcal{R} e \kappa_{1} \kappa_{6}\left(\frac{\|B\|_{1}}{p-1}+\kappa_{1}^{p^{\prime}}\right)^{\frac{2-p}{p}}\right)\left\|D^{\star}(\boldsymbol{u}-\boldsymbol{v})\right\|_{p, B}^{2} \leq 0
$$

and thus $\boldsymbol{u}=\boldsymbol{v}$ if condition (4.14) is satisfied.

Proof of Corollary 4.6. Arguing as in the shear-thickening case (cf. the proof of Corollary 4.5), we can prove that

$$
\|\pi\|_{p^{\prime}} \leq C\left(\|D u\|_{p, B}^{2}+\delta\left\|\nabla u_{3}\right\|_{p, B}^{2}+\left\|\tau\left(D^{\star} \boldsymbol{u}\right)\right\|_{p^{\prime}}+\left\|\nabla^{\star} \cdot \tau\left(D^{\star} \boldsymbol{u}\right)-\nabla \cdot \tau\left(D^{\star} \boldsymbol{u}\right)\right\|_{p^{\prime}}\right)
$$

where $\tau=\left(\tau_{i j}\right)_{i, j=1,2}$. On the other hand, by taking into account (6.5) and (6.10), we obtain

$$
\left\|\tau\left(D^{\star} \boldsymbol{u}\right)\right\|_{p^{\prime}}=2\left\|\left(1+\left|D^{\star} \boldsymbol{u}\right|^{2}\right)^{\frac{p-2}{2}} D u\right\|_{p^{\prime}} \leq 2\|D u\|_{p}^{p-1}
$$

and

$$
\left\|\nabla^{\star} \cdot \tau\left(D^{\star} \boldsymbol{u}\right)-\nabla \cdot \tau\left(D^{\star} \boldsymbol{u}\right)\right\|_{p^{\prime}} \leq 4 \delta m\left(2\|D u\|_{p}^{p-1}+\left\|\frac{\delta}{B} u_{2}\right\|_{p}^{p-1}\right) .
$$

The conclusion follows by combining the three inequalities.

\section{$6.4 \delta$-approximation}

Proof of Proposition 4.11. Even though the idea is similar to the one used in the shearthickening case, the lack of coercivity of the stress tensor when splitting the system and considering the equations for $\left(0,0, w_{3}\right)$ and $\left(w_{1}, w_{2}, 0\right)$ generates an additional difficulty. Setting $\phi=\left(0,0, w_{3}\right)$ in the corresponding weak formulation, we obtain

$$
\int_{\Sigma}\left(1+|D \boldsymbol{w}|^{2}\right)^{\frac{p-2}{2}}\left|\nabla w_{3}\right|^{2} d x=\left(\frac{G}{B}, w_{3}\right)
$$

Let

$$
H_{1}=\left(1+|D \boldsymbol{w}|^{2}\right)^{\frac{2-p}{2}}, \quad H_{2}=\left(1+|D \boldsymbol{w}|^{2}\right)^{\frac{p-2}{2}}\left|\nabla w_{3}\right|^{2}, \quad H_{3}=\left|\nabla w_{3}\right| .
$$

Due to Lemma 6.3, we have

$$
\frac{\left\|\nabla w_{3}\right\|_{p}^{2}}{\left(|\Sigma|+\|D \boldsymbol{w}\|_{p}^{p}\right)^{\frac{2-p}{p}}} \leq\left(\frac{G}{B}, w_{3}\right) .
$$

On the other hand, standard arguments together with the Poincaré inequality (3.9) and the Korn inequality give

$$
\left|\left(\frac{G}{B}, w_{3}\right)\right| \leq c_{1}\left\|\nabla w_{3}\right\|_{p},
$$

where $c_{1}=m|G||\Sigma|^{\frac{1}{p^{\prime}}}$. Combining these inequalities yields

$$
\frac{\left\|\nabla w_{3}\right\|_{p}}{\left(|\Sigma|+\|D \boldsymbol{w}\|_{p}^{p}\right)^{\frac{2-p}{p}}} \leq c_{1} .
$$

Similarly, by setting $\boldsymbol{\varphi}=(w, 0)=\left(w_{1}, w_{2}, 0\right)$ in the corresponding weak formulation, we obtain

$$
2 \int_{\Sigma}\left(1+|D \boldsymbol{w}|^{2}\right)^{\frac{p-2}{2}}|D w|^{2} d x=\delta\left(\sigma\left(w_{3}\right), w_{2}\right)
$$


and thus

$$
\frac{\|D w\|_{p}^{2}}{\left(|\Sigma|+\|D \boldsymbol{w}\|_{p}^{p}\right)^{\frac{2-p}{p}}} \leq \frac{\delta}{2}\left(\sigma\left(w_{3}\right), w_{2}\right) .
$$

Estimate (3.15) together with the Korn inequality give

$$
\begin{aligned}
\frac{\|D w\|_{p}^{2}}{\left(|\Sigma|+\|D \boldsymbol{w}\|_{p}^{p}\right)^{\frac{2-p}{p}}} & \leq \frac{D_{p, \alpha}}{2} c_{0} \delta\left\|\nabla w_{3}\right\|_{p}^{\alpha}\left\|\nabla w_{2}\right\|_{p} \\
& \leq \frac{D_{p, \alpha}}{2} c_{0} \delta\left\|\nabla w_{3}\right\|_{p}^{\alpha}\|\nabla w\|_{p} \leq c_{2} c_{0} \delta\left\|\nabla w_{3}\right\|_{p}^{\alpha}\|D w\|_{p}
\end{aligned}
$$

where $c_{2}=\frac{D_{p, \alpha}}{2 C_{K, 1}}$. Consequently, we have

$$
\frac{\|D w\|_{p}}{\left(|\Sigma|+\|D \boldsymbol{w}\|_{p}^{p}\right)^{\frac{2-p}{p}}} \leq c_{0} c_{2} \delta\left\|\nabla w_{3}\right\|_{p}^{\alpha}
$$

Combining (6.24) and (6.25), it follows that

$$
\begin{aligned}
\frac{\|D \boldsymbol{w}\|_{p}^{p}}{\left(|\Sigma|+\|D \boldsymbol{w}\|_{p}^{p}\right)^{2-p}} & \leq c_{1}^{p}+\left(c_{0} c_{2}\right)^{p}\left\|\nabla w_{3}\right\|_{p}^{\alpha p} \\
& \leq c_{1}^{p}+\left(c_{0} c_{1}^{\alpha} c_{2}\right)^{p}\left(|\Sigma|+\|D \boldsymbol{w}\|_{p}^{p}\right)^{(2-p) \alpha}
\end{aligned}
$$

yielding to

$$
\begin{aligned}
\|D \boldsymbol{w}\|_{p}^{p} & \leq c_{1}^{p}\left(|\Sigma|+\|D \boldsymbol{w}\|_{p}^{p}\right)^{2-p}+\left(c_{0} c_{1}^{\alpha} c_{2}\right)^{p}\left(|\Sigma|+\|D \boldsymbol{w}\|_{p}^{p}\right)^{(2-p)(\alpha+1)} \\
& \leq c_{3}^{p}\left(1+\|D \boldsymbol{w}\|_{p}^{p}\right)^{(2-p)(\alpha+1)}
\end{aligned}
$$

and

$$
\|D \boldsymbol{w}\|_{p}^{\frac{p}{(2-p)(\alpha+1)}} \leq c_{3}^{\frac{p}{(2-p)(\alpha+1)}}\left(1+\|D \boldsymbol{w}\|_{p}^{p}\right),
$$

where $c_{3}=\left(c_{1}+c_{0} c_{1}^{\alpha} c_{2}\right)(1+|\Sigma|)^{\frac{(2-p)(\alpha+1)}{p}}$. By using the Young inequality, we deduce that for $\alpha<\frac{p-1}{2-p}$ we have

$$
\begin{aligned}
c_{3}^{\frac{p}{(2-p)(\alpha+1)}}\|D \boldsymbol{w}\|_{p}^{p} & \leq(2-p)(\alpha+1)\|D \boldsymbol{u}\|_{p}^{\frac{p}{(2-p)(\alpha+1)}} \\
& +(1-(2-p)(\alpha+1)) c_{3}^{\frac{p}{(1-(2-p)(\alpha+1)(2-p)(\alpha+1)}} .
\end{aligned}
$$

Combining (6.26) and (6.27), we obtain

$$
\|D \boldsymbol{w}\|_{p} \leq c_{3}\left(\frac{1}{1-(2-p)(\alpha+1)}+c_{3}^{\frac{1}{1-(2-p)(\alpha+1)}}\right)^{\frac{(2-p)(\alpha+1)}{p}} .
$$

The conclusion follows from (6.24), (6.25) and (6.28).

Proof of Proposition 4.12, The ideas of the proof are similar to the ones used in the shearthickening case. Indeed, by arguing as in the proof of Proposition 4.10, we obtain

$$
\begin{aligned}
(\boldsymbol{\tau}(D \boldsymbol{u})-\boldsymbol{\tau}(D \boldsymbol{w}), D \boldsymbol{\varphi}) & =\left(\boldsymbol{\tau}(D \boldsymbol{u})-\boldsymbol{\tau}\left(D^{\star} \boldsymbol{u}\right), D \boldsymbol{\varphi}\right)+\left(\nabla^{\star} \cdot \boldsymbol{\tau}\left(D^{\star} \boldsymbol{u}\right)-\nabla \cdot \boldsymbol{\tau}\left(D^{\star} \boldsymbol{u}\right), \boldsymbol{\varphi}\right) \\
& -\mathcal{R} e\left(a_{\star}(\boldsymbol{u}, \boldsymbol{u}, \boldsymbol{\varphi})-a(\boldsymbol{w}, \boldsymbol{w}, \boldsymbol{\varphi})\right)+\left(\pi_{1}-\pi_{2}, \nabla \cdot \boldsymbol{\varphi}\right)-\delta\left(\sigma\left(w_{3}\right), \varphi_{2}\right) \\
& =I_{1}+I_{2}+I_{3}+I_{4}+I_{5} .
\end{aligned}
$$


○ By taking into account (6.6), we have

$$
\begin{aligned}
\left|I_{1}\right| & \leq\left\|\boldsymbol{\tau}\left(D^{\star} \boldsymbol{u}\right)-\boldsymbol{\tau}(D \boldsymbol{u})\right\|_{p^{\prime}}\|D \boldsymbol{\varphi}\|_{p} \\
& \leq 2 C_{p}\left\|D^{\star} \boldsymbol{u}-D \boldsymbol{u}\right\|_{p}^{p-1}\|D \boldsymbol{\varphi}\|_{p} \leq F_{1} \delta^{p-1}\|D \boldsymbol{\varphi}\|_{p}
\end{aligned}
$$

where $F_{1}=2 C_{p} m^{p-1}\left(\left\|u_{2}\right\|_{p}+\left\|u_{3}\right\|_{p}\right)^{p-1}$.

$\circ$ Estimate (6.10) together with the Sobolev inequality (3.8) and the Korn inequality (6.1) yield

$$
\begin{aligned}
\left|I_{2}\right| & \leq\left\|\nabla^{\star} \cdot \boldsymbol{\tau}\left(D^{\star} \boldsymbol{u}\right)-\nabla \cdot \boldsymbol{\tau}\left(D^{\star} \boldsymbol{u}\right)\right\|_{p^{\prime}}\|\boldsymbol{\varphi}\|_{p} \\
& \leq 4 m S_{p, p}\left(\|D u\|_{p}^{p-1}+\left\|D_{33}^{\star} \boldsymbol{u}\right\|_{p}^{p-1}+\left\|D_{23}^{\star} \boldsymbol{u}\right\|_{p}^{p-1}\right) \delta\|\nabla \boldsymbol{\varphi}\|_{p} \\
& \leq 12 m S_{p, p}\left\|D^{\star} \boldsymbol{u}\right\|_{p}^{p-1} \delta\|\nabla \boldsymbol{\varphi}\|_{p} \leq F_{2} \delta\|D \boldsymbol{\varphi}\|_{p}
\end{aligned}
$$

where $F_{2}=\frac{12 m}{C_{K, 1}} S_{p, p}\left\|D^{\star} \boldsymbol{u}\right\|_{p}^{p-1}$.

$\circ$ The convective term is estimated similarly

$$
\begin{aligned}
\frac{1}{\mathcal{R} e}\left|I_{3}\right| & =\left|a_{\star}(\boldsymbol{u}, \boldsymbol{u}, \boldsymbol{\varphi})-a(\boldsymbol{w}, \boldsymbol{w}, \boldsymbol{\varphi})\right| \\
& \leq\left|a_{\star}(\boldsymbol{u}-\boldsymbol{w}, \boldsymbol{u}, \boldsymbol{\varphi})\right|+|a(\boldsymbol{w}, \boldsymbol{u}-\boldsymbol{w}, \boldsymbol{\varphi})|+\left|\left(\boldsymbol{w} \cdot \nabla^{\star} \boldsymbol{u}-\boldsymbol{w} \cdot \nabla \boldsymbol{u}, \boldsymbol{\varphi}\right)\right| \\
& \leq\left|a_{\star}(\boldsymbol{u}-\boldsymbol{w}, \boldsymbol{u}, \boldsymbol{\varphi})\right|+|a(\boldsymbol{w}, \boldsymbol{u}-\boldsymbol{w}, \boldsymbol{\varphi})|+\left\|\frac{\delta}{B} \mid \boldsymbol{w}\right\| \boldsymbol{u}\|\boldsymbol{\varphi}\|_{1} \\
& \leq\|\boldsymbol{u}-\boldsymbol{w}\|_{2 p^{\prime}}\left\|\nabla^{\star} \boldsymbol{u}\right\|_{p}\|\boldsymbol{\varphi}\|_{2 p^{\prime}}+\|\boldsymbol{w}\|_{2 p^{\prime}}\|\nabla(\boldsymbol{u}-\boldsymbol{w})\|_{p}\|\boldsymbol{\varphi}\|_{2 p^{\prime}}+\delta m\|\boldsymbol{w}\|_{2 p^{\prime}}\|\boldsymbol{u}\|_{2 p^{\prime}}\|\boldsymbol{\varphi}\|_{p} \\
& \leq\left(1+S_{p, p}\right)\left(S_{p, 2 p^{\prime}}\right)^{2}\left(\|\nabla(\boldsymbol{u}-\boldsymbol{w})\|_{p}\left(\left\|\nabla^{\star} \boldsymbol{u}\right\|_{p}+\|\nabla \boldsymbol{w}\|_{p}\right)+\delta m\|\nabla \boldsymbol{w}\|_{p}\|\nabla \boldsymbol{u}\|_{p}\right)\|\nabla \boldsymbol{\varphi}\|_{p} \\
& \leq F_{3}\left(\delta+\|D(\boldsymbol{u}-\boldsymbol{w})\|_{p}\right)\|D \boldsymbol{\varphi}\|_{p}
\end{aligned}
$$

with $F_{3}=\frac{1+m}{C_{K}^{3}}\left(1+S_{p, p}\right)\left(S_{p, 2 p^{\prime}}\right)^{2}\left(\left\|D^{\star} \boldsymbol{u}\right\|_{p}+\|D \boldsymbol{w}\|_{p}+\|D \boldsymbol{u}\|_{p}\|D \boldsymbol{w}\|_{p}\right)$, and where $C_{K}$ is the Korn constant given in (6.1).

- The estimate associated to $\left|I_{4}\right|$ may be obtained with slight modifications in the proof given in Corollary 4.5 by observing that

$$
\begin{aligned}
\left\|\pi_{1}-\pi_{2}\right\|_{p^{\prime}} & \leq\left\|\tau\left(D^{\star} \boldsymbol{u}\right)-\tau(D \boldsymbol{w})\right\|_{p^{\prime}}+\left\|\nabla^{\star} \cdot \tau\left(D^{\star} \boldsymbol{u}\right)-\nabla^{\star} \cdot \tau\left(D^{\star} \boldsymbol{u}\right)\right\|_{p^{\prime}} \\
& +\mathcal{R} e\left(\|u \otimes u-w \otimes w\|_{p^{\prime}}+\delta\left\|u_{3}^{2}\right\|_{p^{\prime}}\right)+\delta\left\|\sigma\left(w_{3}\right)\right\|_{p^{\prime}} .
\end{aligned}
$$

Taking into account (6.23) and (6.6), we have

$$
\left\|\nabla^{\star} \cdot \tau\left(D^{\star} \boldsymbol{u}\right)-\nabla^{\star} \cdot \tau\left(D^{\star} \boldsymbol{u}\right)\right\|_{p^{\prime}} \leq F_{4,1} \delta
$$

and

$$
\begin{aligned}
\left\|\tau\left(D^{\star} \boldsymbol{u}\right)-\tau(D \boldsymbol{w})\right\|_{p^{\prime}, B} & \leq\left\|\tau\left(D^{\star} \boldsymbol{u}\right)-\tau(D \boldsymbol{w})\right\|_{p^{\prime}} \leq 2 C_{p}\left\|D^{\star} \boldsymbol{u}-D \boldsymbol{w}\right\|_{p}^{p-1} \\
& \leq 2 C_{p}\left(\left\|D^{\star} \boldsymbol{u}-D \boldsymbol{u}\right\|_{p}^{p-1}+\|D \boldsymbol{u}-D \boldsymbol{w}\|_{p}^{p-1}\right) \\
& \leq F_{4,2}\left(\|D \boldsymbol{u}-D \boldsymbol{w}\|_{p}^{p-1}+\delta^{p-1}\right)
\end{aligned}
$$


where $F_{4,2}=2 C_{p}+F_{1}$. Moreover, by using the Sobolev inequality (3.8) with $(q, r)=(2,4)$ and the classical Korn inequality, we obtain

$$
\begin{aligned}
\|u \otimes u-w \otimes w\|_{p^{\prime}}+\delta\left\|u_{3}^{2}\right\|_{p^{\prime}} & \leq\|(u+w) \otimes(u-w)\|_{p^{\prime}}+\delta\left\|u_{3}\right\|_{2 p^{\prime}}^{2} \\
& \leq\|u+w\|_{2 p^{\prime}}\|u-w\|_{2 p^{\prime}}+\delta\left\|u_{3}\right\|_{2 p^{\prime}}^{2} \\
& \leq S_{p, 2 p^{\prime}}\|\nabla(u-w)\|_{p}\|u+w\|_{2 p^{\prime}}+\delta\left\|u_{3}\right\|_{2 p^{\prime}}^{2} \\
& \leq S_{p, 2 p^{\prime}}\|\nabla(\boldsymbol{u}-\boldsymbol{w})\|_{p}\|u+w\|_{2 p^{\prime}}+\delta\left\|u_{3}\right\|_{2 p^{\prime}}^{2} \\
& \leq \frac{S_{p, 2 p^{\prime}}}{C_{K}}\|D(\boldsymbol{u}-\boldsymbol{w})\|_{p}\|u+w\|_{2 p^{\prime}}+\delta\left\|u_{3}\right\|_{2 p^{\prime}}^{2} \\
& \leq F_{4,3}\left(\|D(\boldsymbol{u}-\boldsymbol{w})\|_{p}+\delta\right),
\end{aligned}
$$

where $F_{4,3}=\frac{S_{p, 2 p^{\prime}}}{C_{K, 1}}\|u+w\|_{2 p^{\prime}}+\left\|u_{3}\right\|_{2 p^{\prime}}^{2}$. Due to (3.16), we have

$$
\left\|\sigma\left(w_{3}\right)\right\|_{p^{\prime}} \leq c_{0} E_{\alpha, p}\left\|\nabla w_{3}\right\|_{p}^{\alpha}=F_{4,4} .
$$

Combining these estimates, we deduce that

$$
\begin{aligned}
\left|I_{4}\right| & \leq\left(F_{4,2}\|D \boldsymbol{u}-D \boldsymbol{w}\|_{p}^{p-1}+\mathcal{R} e F_{4,3}\|D \boldsymbol{u}-D \boldsymbol{w}\|_{p}\right)\|\nabla \cdot \boldsymbol{\varphi}\|_{p} \\
& +\left(\left(F_{4,1}+\mathcal{R} e F_{4,3}\right) \delta+F_{5,2} \delta^{p-1}\right)\|\nabla \cdot \boldsymbol{\varphi}\|_{p} \\
& \leq F_{4}\left(\|D(\boldsymbol{u}-\boldsymbol{w})\|_{p}+\|D(\boldsymbol{u}-\boldsymbol{w})\|_{p}^{p-1}+\delta^{p-1}\right)\|\nabla \cdot \boldsymbol{\varphi}\|_{p} .
\end{aligned}
$$

$\circ$ Finally, taking into account (3.15) we obtain

$$
\begin{aligned}
\left|I_{5}\right| & =\delta\left|\left(\sigma\left(w_{3}\right), \varphi_{2}\right)\right| \leq D_{p, \alpha} \delta\left\|\nabla w_{3}\right\|_{p}^{\alpha}\left\|\nabla \varphi_{2}\right\|_{p} \\
& \leq D_{p, \alpha} \delta\left\|\nabla w_{3}\right\|_{2}^{\alpha}\|\nabla \varphi\|_{p} \leq F_{5} \delta\|D \varphi\|_{p},
\end{aligned}
$$

where $F_{5}=\frac{D_{p, \alpha}}{C_{K, 1}}\left\|\nabla w_{3}\right\|_{p}^{\alpha}$.

$\circ$ Combining (6.30)-(6.34), and taking into the estimates associated to $\boldsymbol{u}$ and $\boldsymbol{w}$, we deduce that

$$
\begin{aligned}
& |(\boldsymbol{\tau}(D \boldsymbol{u})-\boldsymbol{\tau}(D \boldsymbol{w}), D \boldsymbol{\varphi})| \\
& \leq\left(F_{1} \delta^{p-1}+F_{2} \delta+\mathcal{R} e F_{3} \delta+F_{5} \delta\right)\|D(\boldsymbol{u}-\boldsymbol{w})\|_{p}+\mathcal{R} e F_{3}\|D(\boldsymbol{u}-\boldsymbol{w})\|_{p}\|D \boldsymbol{\varphi}\|_{p} \\
& +F_{4}\left(\|D(\boldsymbol{u}-\boldsymbol{w})\|_{p}+\|D(\boldsymbol{u}-\boldsymbol{w})\|_{p}^{p-1}+\delta^{p-1}\right)\|\nabla \cdot \boldsymbol{\varphi}\|_{p} \\
& \leq C_{1}\left(\delta^{p-1}\left\|D^{\star}(\boldsymbol{u}-\boldsymbol{w})\right\|_{p, B}+\delta\left\|D^{\star}(\boldsymbol{u}-\boldsymbol{w})\right\|_{p, B}^{p-1}+\mathcal{R} e\left\|D^{\star}(\boldsymbol{u}-\boldsymbol{w})\right\|_{p, B}^{2}+\delta^{p}\right),
\end{aligned}
$$

where $C_{1}$ depends only on $p, \Sigma, m, n, \alpha$ and $c_{0}$. Setting $\boldsymbol{\varphi}=\boldsymbol{u}-\boldsymbol{w}$ and taking into account the estimates associated to $\boldsymbol{u}$ and $\boldsymbol{w}$, we deduce that

$$
\begin{aligned}
& (\boldsymbol{\tau}(D \boldsymbol{u})-\boldsymbol{\tau}(D \boldsymbol{w}), D(\boldsymbol{u}-\boldsymbol{w})) \\
& \leq\left(F_{1} \delta^{p-1}+F_{2} \delta+\mathcal{R} e F_{3} \delta+F_{5} \delta\right)\|D(\boldsymbol{u}-\boldsymbol{w})\|_{p}+\mathcal{R} e F_{3}\|D(\boldsymbol{u}-\boldsymbol{w})\|_{p}^{2} \\
& +F_{4}\left(\|D(\boldsymbol{u}-\boldsymbol{w})\|_{p}+\|D(\boldsymbol{u}-\boldsymbol{w})\|_{p}^{p-1}+\delta^{p-1}\right)\left\|\frac{\delta}{B} u_{2}\right\|_{p} \\
& \leq C_{2}\left(\delta^{p-1}\|D(\boldsymbol{u}-\boldsymbol{w})\|_{p}+\delta\|D(\boldsymbol{u}-\boldsymbol{w})\|_{p}^{p-1}+\mathcal{R} e\|D(\boldsymbol{u}-\boldsymbol{w})\|_{p}^{2}+\delta^{p}\right),
\end{aligned}
$$


where $C_{2}$ depends only on $p, \Sigma, m, n, \alpha$ and $c_{0}$. Using the Young inequality, it follows that for every $\varepsilon>0$, we have

$$
\begin{aligned}
& (\boldsymbol{\tau}(D \boldsymbol{u})-\boldsymbol{\tau}(D \boldsymbol{w}), D(\boldsymbol{u}-\boldsymbol{w})) \\
& \leq\left(\varepsilon+C_{2} \mathcal{R} e\right)\left\|D^{\star} \boldsymbol{u}-D^{\star} \boldsymbol{w}\right\|_{p, B}^{2}+C_{3}(\varepsilon)\left(\delta^{2(p-1)}+\delta^{\frac{2}{3-p}}\right)+C_{1} \delta^{p} .
\end{aligned}
$$

On the other hand, by taking into account (6.8) and the estimates associated to $\boldsymbol{u}$ and $\boldsymbol{w}$, we deduce that there exists a constant $C_{4}$ depending on $p, \Sigma, G$ and $m, \alpha$ and $c_{0}$, but independent of $\delta$, such that

$$
(\boldsymbol{\tau}(D \boldsymbol{u})-\boldsymbol{\tau}(D \boldsymbol{w}), D(\boldsymbol{u}-\boldsymbol{w})) \geq \frac{2\|D(\boldsymbol{u}-\boldsymbol{w})\|_{p}^{2}}{\left(|\Sigma|+\|D \boldsymbol{u}\|_{p}^{p}+\|D \boldsymbol{w}\|_{p}\right)^{\frac{2-p}{p}}} \geq C_{4}\|D(\boldsymbol{u}-\boldsymbol{w})\|_{p}^{2} .
$$

Combining (6.35) and (6.36), observing that $\delta^{p}<\delta^{2(p-1)}$ and $\delta^{\frac{2}{3-p}}<\delta^{2(p-1)}$, choosing $\varepsilon=\frac{C_{4}}{2}$ and assuming that $\mathcal{R} e<\frac{C_{4}}{2 C_{2}}$, we deduce that

$$
\|D(\boldsymbol{u}-\boldsymbol{w})\|_{p, B} \leq C_{5} \delta^{p-1}
$$

and the claimed result is proved.

\section{A Toroidal coordinate system}

Let us consider the new coordinate system, in the variables $\left(\widetilde{x}_{1}, \widetilde{x}_{2}, \widetilde{x}_{3}\right)$, given by the transformations $\left(\widetilde{x}_{1}, \widetilde{x}_{2}, \widetilde{x}_{3}\right) \mapsto\left(\widetilde{y}_{1}, \widetilde{y}_{2}, \widetilde{y}_{3}\right)$ satisfying (2.2) and (2.3). Let $M$ be a generic point such that

$$
M=\widetilde{y}_{1}\left(\widetilde{x}_{1}, \widetilde{x}_{2}, \widetilde{x}_{3}\right) \mathbf{e}_{1}+\widetilde{y}_{2}\left(\widetilde{x}_{1}, \widetilde{x}_{2}, \widetilde{x}_{3}\right) \mathbf{e}_{2}+\widetilde{y}_{3}\left(\widetilde{x}_{1}, \widetilde{x}_{2}, \widetilde{x}_{3}\right) \mathbf{e}_{3} .
$$

Defining the scale vectors

$$
h_{1}=\left|\frac{\partial M}{\partial \widetilde{x}_{1}}\right|, \quad h_{2}=\left|\frac{\partial M}{\partial \widetilde{x}_{2}}\right|, \quad h_{3}=\left|\frac{\partial M}{\partial \widetilde{x}_{3}}\right|
$$

and the local basis

$$
\mathbf{a}_{1}=\frac{1}{h_{1}} \frac{\partial M}{\partial \widetilde{x}_{1}}, \quad \mathbf{a}_{2}=\frac{1}{h_{2}} \frac{\partial M}{\partial \widetilde{x}_{2}}, \quad \mathbf{a}_{3}=\frac{1}{h_{3}} \frac{\partial M}{\partial \widetilde{x}_{3}},
$$

we obtain,

$$
h_{1}=1, \quad h_{2}=1, \quad h_{3}=1+\frac{1}{R} \widetilde{x}_{2}
$$

and

$$
\mathbf{a}_{1}=\mathbf{e}_{3}, \quad \mathbf{a}_{2}=\cos \left(\frac{\widetilde{x}_{3}}{R}\right) \mathbf{e}_{1}+\sin \left(\frac{\widetilde{x}_{3}}{R}\right) \mathbf{e}_{2}, \quad \mathbf{a}_{3}=-\sin \left(\frac{\widetilde{x}_{3}}{R}\right) \mathbf{e}_{1}+\cos \left(\frac{\widetilde{x}_{3}}{R}\right) \mathbf{e}_{2} .
$$

The corresponding derivatives of the vector basis are given by

$$
\begin{array}{lllrl}
\frac{\partial \mathbf{a}_{1}}{\partial \widetilde{x}_{1}}=0, & \frac{\partial \mathbf{a}_{1}}{\partial \widetilde{x}_{2}}=0, & & \frac{\partial \mathbf{a}_{1}}{\partial \widetilde{x}_{3}}=0, \\
\frac{\partial \mathbf{a}_{2}}{\partial \widetilde{x}_{1}}=0, & \frac{\partial \mathbf{a}_{2}}{\partial \widetilde{x}_{2}}=0, & & \frac{\partial \mathbf{a}_{2}}{\partial \widetilde{x}_{3}}=\frac{\mathbf{a}_{3}}{R}, \\
\frac{\partial \mathbf{a}_{3}}{\partial \widetilde{x}_{1}}=0, & \frac{\partial \mathbf{a}_{3}}{\partial \widetilde{x}_{2}}=0, & & \frac{\partial \mathbf{a}_{3}}{\partial \widetilde{x}_{3}}=-\frac{\mathbf{a}_{2}}{R} .
\end{array}
$$

- The gradient of a scalar function $\widetilde{\psi}$ in the rectangular toroidal coordinates is then given by

$$
\widetilde{\nabla} \widetilde{\psi}=\frac{1}{h_{1}} \frac{\partial \widetilde{\psi}}{\partial \widetilde{x}_{1}} \mathbf{a}_{1}+\frac{1}{h_{2}} \frac{\partial \widetilde{\psi}}{\partial \widetilde{x}_{2}} \mathbf{a}_{2}+\frac{1}{h_{3}} \frac{\partial \widetilde{\psi}}{\partial \widetilde{x}_{3}} \mathbf{a}_{3}=\frac{\partial \widetilde{\psi}}{\partial \widetilde{x}_{1}} \mathbf{a}_{1}+\frac{\partial \widetilde{\psi}}{\partial \widetilde{x}_{2}} \mathbf{a}_{2}+\frac{1}{B} \frac{\partial \widetilde{\psi}}{\partial \widetilde{x}_{3}} \mathbf{a}_{3}
$$


where $B=1+\frac{1}{R} \widetilde{x}_{2}$.

- The gradient of a vector $\widetilde{\boldsymbol{v}} \equiv\left(\widetilde{v}_{1}, \widetilde{v}_{2}, \widetilde{v}_{3}\right)$ is defined by

$$
\widetilde{\nabla} \widetilde{\boldsymbol{v}}=\left(\frac{1}{h_{1}} \mathbf{a}_{1} \otimes \frac{\partial}{\partial \widetilde{x}_{1}}+\frac{1}{h_{2}} \mathbf{a}_{2} \otimes \frac{\partial}{\partial \widetilde{x}_{2}}+\frac{1}{h_{3}} \mathbf{a}_{3} \otimes \frac{\partial}{\partial \widetilde{x}_{3}}\right)\left(\mathbf{a}_{1} \widetilde{v}_{1}+\mathbf{a}_{2} \widetilde{v}_{2}+\mathbf{a}_{3} \widetilde{v}_{3}\right)
$$

that is

$$
\begin{aligned}
\widetilde{\nabla} \widetilde{\boldsymbol{v}} & =\sum_{j=1}^{3} \sum_{i=1}^{2} \frac{\partial \widetilde{v}_{j}}{\partial \widetilde{x}_{i}} \mathbf{a}_{i} \otimes \mathbf{a}_{j}+\frac{1}{R B}\left(\widetilde{v}_{2} \mathbf{a}_{3} \otimes \mathbf{a}_{3}-\widetilde{v}_{3} \mathbf{a}_{3} \otimes \mathbf{a}_{2}\right)+\frac{1}{B} \sum_{j=1}^{3} \frac{\partial \widetilde{v}_{j}}{\partial \widetilde{x}_{3}} \mathbf{a}_{3} \otimes \mathbf{a}_{j} \\
& =\left(\begin{array}{lll}
\frac{\partial \widetilde{v}_{1}}{\partial \widetilde{x}_{1}} & \frac{\partial \widetilde{v}_{2}}{\partial \widetilde{x}_{1}} & \frac{\partial \widetilde{v}_{3}}{\partial \widetilde{x}_{1}} \\
\frac{\partial \widetilde{v}_{1}}{\partial \widetilde{x}_{2}} & \frac{\partial \widetilde{v}_{2}}{\partial \widetilde{x}_{2}} & \frac{\partial \widetilde{v}_{3}}{\partial \widetilde{x}_{2}} \\
\frac{1}{B} \frac{\partial \widetilde{v}_{1}}{\partial \widetilde{x}_{3}} & \frac{1}{B}\left(\frac{\partial \widetilde{v}_{2}}{\partial \widetilde{x}_{3}}-\frac{\widetilde{v}_{3}}{R}\right) & \frac{1}{B}\left(\frac{\partial \widetilde{v}_{3}}{\partial \widetilde{x}_{3}}+\frac{\widetilde{v}_{2}}{R}\right)
\end{array}\right) .
\end{aligned}
$$

- Similarly, if $\widetilde{\boldsymbol{v}} \equiv\left(\widetilde{v}_{1}, \widetilde{v}_{2}, \widetilde{v}_{3}\right)$ and $\widetilde{\boldsymbol{w}} \equiv\left(\widetilde{w}_{1}, \widetilde{w}_{2}, \widetilde{w}_{3}\right)$ are two vectors, then the convective term is defined by

$$
\widetilde{\boldsymbol{v}} \cdot \widetilde{\nabla} \widetilde{\boldsymbol{w}}=\left(\mathbf{a}_{1} \widetilde{v}_{1}+\mathbf{a}_{2} \widetilde{v}_{2}+\mathbf{a}_{3} \widetilde{v}_{3}\right) \cdot\left(\sum_{i, j} \mathbf{a}_{i} \otimes \mathbf{a}_{j}(\widetilde{\nabla} \widetilde{\boldsymbol{w}})_{i j}\right)=\sum_{i} \mathbf{a}_{i}\left(\sum_{j} \widetilde{v}_{j}(\widetilde{\nabla} \widetilde{\boldsymbol{w}})_{j i}\right),
$$

that is

$$
\begin{aligned}
\widetilde{\boldsymbol{v}} \cdot \widetilde{\nabla} \widetilde{\boldsymbol{w}}= & \sum_{j=1}^{3} \sum_{i=1}^{2} \widetilde{v}_{i} \frac{\partial \widetilde{w}_{j}}{\partial \widetilde{x}_{i}} \mathbf{a}_{j}+\frac{\widetilde{v}_{3}}{R B}\left(\widetilde{w}_{2} \mathbf{a}_{3}-\widetilde{w}_{3} \mathbf{a}_{2}\right)+\frac{\widetilde{v}_{3}}{B} \frac{\partial \widetilde{\boldsymbol{w}}}{\partial \widetilde{x}_{3}} \\
= & \left(\begin{array}{c}
\widetilde{v}_{1} \frac{\partial \widetilde{w}_{1}}{\partial \widetilde{x}_{1}}+\widetilde{v}_{2} \frac{\partial \widetilde{w}_{1}}{\partial \widetilde{x}_{2}}+\frac{\widetilde{v}_{3}}{B} \frac{\partial \widetilde{w}_{1}}{\partial \widetilde{x}_{3}} \\
\widetilde{v}_{1} \frac{\partial \widetilde{\widetilde{w}}_{2}}{\partial \widetilde{x}_{1}}+\widetilde{v}_{2} \frac{\partial \widetilde{\widetilde{x}}_{2}}{\partial \widetilde{x}_{2}}+\frac{\widetilde{v}_{3}}{B} \frac{\partial \widetilde{w}_{2}}{\partial \widetilde{x}_{3}}-\frac{1}{R B} \widetilde{v}_{3} \widetilde{w}_{3} \\
\widetilde{v}_{1} \frac{\partial \widetilde{w}_{3}}{\partial \widetilde{x}_{1}}+\widetilde{v}_{2} \frac{\partial \widetilde{w}_{3}}{\partial \widetilde{x}_{2}}+\frac{\widetilde{v}_{3}}{B} \frac{\partial \widetilde{w}_{3}}{\partial \widetilde{x}_{3}}+\frac{1}{R B} \widetilde{v}_{3} \widetilde{w}_{2}
\end{array}\right) .
\end{aligned}
$$

- Finally, the divergence of a vector $\widetilde{\boldsymbol{v}} \equiv\left(\widetilde{v}_{1}, \widetilde{v}_{2}, \widetilde{v}_{3}\right)$ is defined by

$$
\begin{aligned}
\widetilde{\nabla} \cdot \widetilde{\boldsymbol{v}} & =\frac{1}{h_{1} h_{2} h_{3}}\left(\frac{\partial}{\partial \widetilde{x}_{1}}\left(h_{2} h_{3} \widetilde{v}_{1}\right)+\frac{\partial}{\partial \widetilde{x}_{2}}\left(h_{3} h_{1} \widetilde{v}_{2}\right)+\frac{\partial}{\partial \widetilde{x}_{3}}\left(h_{1} h_{2} \widetilde{v}_{3}\right)\right) \\
& =\frac{\partial \widetilde{v}_{1}}{\partial \widetilde{x}_{1}}+\frac{\partial \widetilde{v}_{2}}{\partial \widetilde{x}_{2}}+\frac{\widetilde{v}_{2}}{R B}+\frac{1}{B} \frac{\partial \widetilde{v}_{3}}{\partial \widetilde{x}_{3}} .
\end{aligned}
$$

The divergence of a tensor $\widetilde{\mathbf{S}}$ is defined by

$$
\widetilde{\nabla} \cdot \widetilde{\mathbf{S}}=\left(\mathbf{a}_{1} \frac{\partial}{\partial \widetilde{x}_{1}}+\mathbf{a}_{2} \frac{\partial}{\partial \widetilde{x}_{2}}+\mathbf{a}_{3} \frac{1}{B} \frac{\partial}{\partial \widetilde{x}_{3}}\right) \cdot\left(\sum_{i, j} \mathbf{a}_{i} \otimes \mathbf{a}_{j} \widetilde{S}_{i j}\right)
$$

which gives

$$
\begin{aligned}
\widetilde{\nabla} \cdot \widetilde{\mathbf{S}}= & \sum_{j=1}^{3} \sum_{i=1}^{2} \frac{\partial \widetilde{S}_{i j}}{\partial \widetilde{x}_{i}} \mathbf{a}_{j}+\frac{1}{B} \sum_{j=1}^{3} \frac{\partial \widetilde{S}_{3 j}}{\partial \widetilde{x}_{3}} \mathbf{a}_{j}+\frac{1}{R B}\left(\widetilde{S}_{21} \mathbf{a}_{1}+\left(\widetilde{S}_{22}-\widetilde{S}_{33}\right) \mathbf{a}_{2}+\left(\widetilde{S}_{32}+\widetilde{S}_{23}\right) \mathbf{a}_{3}\right) \\
= & \left(\begin{array}{l}
\frac{\partial \widetilde{S}_{11}}{\partial \widetilde{x}_{1}}+\frac{\partial \widetilde{S}_{21}}{\partial \widetilde{x}_{2}}+\frac{1}{B} \frac{\partial \widetilde{S}_{31}}{\partial \widetilde{x}_{3}}+\frac{\widetilde{S}_{21}}{R B} \\
\frac{\partial \widetilde{S}_{12}}{\partial \widetilde{x}_{1}}+\frac{\partial \widetilde{S}_{\widetilde{x}_{2}}}{\partial \widetilde{x}_{2}}+\frac{1}{B} \frac{\partial \widetilde{S}_{32}}{\partial \widetilde{x}_{3}}+\frac{\widetilde{S}_{22}-\widetilde{S}_{33}}{R B} \\
\frac{\partial \widetilde{S}_{13}}{\partial \widetilde{x}_{1}}+\frac{\partial \widetilde{S}_{23}}{\partial \widetilde{x}_{2}}+\frac{1}{B} \frac{\partial \widetilde{S}_{33}}{\partial \widetilde{x}_{3}}+\frac{\widetilde{S}_{32}+\widetilde{S}_{23}}{R B}
\end{array}\right)
\end{aligned}
$$




\section{B Dimensionless system}

For the confort of the reader, the dimensionless equation (2.7) is derived hereafter. To simplify the notation, we consider the fully developed case and will assume that (2.4) and (2.5) are fulfilled. Taking into account (A.3), (A.1) and (2.6), we can easily see that

$$
\begin{aligned}
& \widetilde{\boldsymbol{u}} \cdot \widetilde{\nabla} \widetilde{\boldsymbol{u}}=\left(\begin{array}{l}
\widetilde{u}_{1} \frac{\partial \widetilde{u}_{1}}{\partial \widetilde{x}_{1}}+\widetilde{u}_{2} \frac{\partial \widetilde{u}_{1}}{\partial \widetilde{x}_{2}} \\
\widetilde{u}_{1} \frac{\partial \widetilde{u}_{2}}{\partial \widetilde{x}_{1}}+\widetilde{u}_{2} \frac{\partial \widetilde{u}_{2}}{\partial \widetilde{x}_{2}}-\frac{1}{R B} \widetilde{u}_{3}^{2} \\
\widetilde{u}_{1} \frac{\partial \widetilde{u}_{3}}{\partial \widetilde{x}_{1}}+\widetilde{u}_{2} \frac{\partial \widetilde{u}_{3}}{\partial \widetilde{x}_{2}}+\frac{1}{R B} \widetilde{u}_{3} \widetilde{u}_{2}
\end{array}\right) \\
& =\frac{U_{0}^{2}}{r_{0}} \underbrace{\left(\begin{array}{l}
u_{1} \frac{\partial u_{1}}{\partial x_{1}}+u_{2} \frac{\partial u_{1}}{\partial x_{2}} \\
u_{1} \frac{\partial u_{2}}{\partial x_{1}}+u_{2} \frac{\partial u_{2}}{\partial x_{2}}-\frac{\delta}{B} u_{3}^{2} \\
u_{1} \frac{\partial u_{3}}{\partial x_{1}}+u_{2} \frac{\partial u_{3}}{\partial x_{2}}+\frac{\delta}{B} u_{3} u_{2}
\end{array}\right)}_{u \cdot \nabla^{\star} \boldsymbol{u}}
\end{aligned}
$$

and

$$
\widetilde{\nabla} \widetilde{\pi}=\left(\begin{array}{c}
\frac{1}{r_{0}} \frac{\partial \widetilde{\pi}}{\partial x_{1}} \\
\frac{1}{r_{0}} \frac{\partial \widetilde{\pi}}{\partial x_{2}} \\
-\frac{\widetilde{G}}{B}
\end{array}\right)=\frac{\mu U_{0}}{r_{0}^{2}} \underbrace{\left(\begin{array}{c}
\frac{\partial \pi}{\partial x_{1}} \\
\frac{\partial \pi}{\partial x_{2}} \\
-\frac{G}{B}
\end{array}\right)}_{\nabla \star \pi}
$$

with $B=1+\delta x_{2}$. Similarly, by taking into account (A.2), we obtain

$$
\begin{aligned}
& \widetilde{D} \widetilde{\boldsymbol{u}}=\left(\begin{array}{lll}
\frac{\partial \widetilde{u}_{1}}{\partial \widetilde{x}_{1}} & \frac{1}{2}\left(\frac{\partial \widetilde{u}_{2}}{\partial \widetilde{x}_{1}}+\frac{\partial \widetilde{u}_{1}}{\partial \widetilde{x}_{2}}\right) & \frac{1}{2} \frac{\partial \widetilde{u}_{3}}{\partial \widetilde{x}_{1}} \\
\frac{1}{2}\left(\frac{\partial \widetilde{u}_{2}}{\partial \widetilde{x}_{1}}+\frac{\partial \widetilde{u}_{1}}{\partial \widetilde{x}_{2}}\right) & \frac{\partial \widetilde{u}_{2}}{\partial \widetilde{x}_{2}} & \frac{1}{2}\left(\frac{\partial \widetilde{u}_{3}}{\partial \widetilde{x}_{2}}-\frac{\widetilde{u}_{3}}{R B}\right) \\
\frac{1}{2} \frac{\partial \widetilde{u}_{3}}{\partial \widetilde{x}_{1}} & \frac{1}{2}\left(\frac{\partial \widetilde{u}_{3}}{\partial \widetilde{x}_{2}}-\frac{\widetilde{u}_{3}}{R B}\right) & \frac{\widetilde{u}_{2}}{R B}
\end{array}\right) \\
& =\frac{U_{0}}{r_{0}} \underbrace{\left(\begin{array}{lll}
\frac{\partial u_{1}}{\partial x_{1}} & \frac{1}{2}\left(\frac{\partial u_{2}}{\partial x_{1}}+\frac{\partial u_{1}}{\partial x_{2}}\right) & \frac{1}{2} \frac{\partial u_{3}}{\partial x_{1}} \\
\frac{1}{2}\left(\frac{\partial u_{2}}{\partial x_{1}}+\frac{\partial u_{1}}{\partial x_{2}}\right) & \frac{\partial u_{2}}{\partial x_{2}} & \frac{1}{2}\left(\frac{\partial u_{3}}{\partial x_{2}}-\frac{\delta}{B} u_{3}\right) \\
\frac{1}{2} \frac{\partial u_{3}}{\partial x_{1}} & \frac{1}{2}\left(\frac{\partial u_{3}}{\partial x_{2}}-\frac{\delta}{B} u_{3}\right) & \frac{\delta}{B} u_{2}
\end{array}\right) .}_{D^{\star} \boldsymbol{u}} .
\end{aligned}
$$

It follows that $|\widetilde{D} \widetilde{\boldsymbol{u}}|^{2}=\left(\frac{U_{0}}{r_{0}}\right)^{2}\left|D^{\star} \boldsymbol{u}\right|^{2}$ and

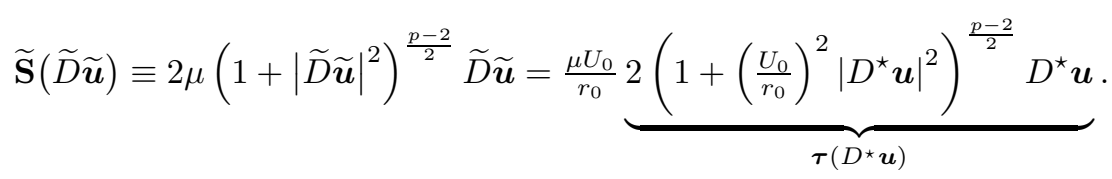


Due to A.5 to the fact that $\widetilde{\mathbf{S}}$ is symmetric and that

$$
\frac{\partial \widetilde{S}_{3 i}}{\partial \widetilde{x}_{3}}=0 \quad i=1,2,3
$$

we deduce that

$$
\begin{aligned}
& \widetilde{\nabla} \cdot(\widetilde{\mathbf{S}}(\widetilde{D} \widetilde{\boldsymbol{u}}))=\left(\begin{array}{l}
\frac{\partial \widetilde{S}_{11}}{\partial \widetilde{x}_{1}}+\frac{\partial \widetilde{S}_{12}}{\partial \widetilde{x}_{2}}+\frac{1}{R B} \widetilde{S}_{12} \\
\frac{\partial \widetilde{S}_{12}}{\partial \widetilde{x}_{1}}+\frac{\partial \widetilde{S}_{22}}{\partial \widetilde{x}_{2}}+\frac{1}{R B}\left(\widetilde{S}_{22}-\widetilde{\tau}_{33}\right) \\
\frac{\partial \widetilde{S}_{13}}{\partial \widetilde{x}_{1}}+\frac{\partial \widetilde{S}_{23}}{\partial \widetilde{x}_{2}}+\frac{2}{R B} \widetilde{S}_{23}
\end{array}\right) \\
& =\frac{1}{r_{0}} \quad\left(\begin{array}{l}
\frac{\partial \widetilde{S}_{11}}{\partial x_{1}}+\frac{\partial \widetilde{S}_{12}}{\partial x_{2}}+\frac{\delta}{B} \widetilde{S}_{12} \\
\frac{\partial \widetilde{S}_{12}}{\partial x_{1}}+\frac{\partial \widetilde{S}_{22}}{\partial x_{2}}+\frac{\delta}{B}\left(\widetilde{S}_{22}-\widetilde{S}_{33}\right) \\
\frac{\partial \widetilde{S}_{13}}{\partial x_{1}}+\frac{\partial \widetilde{S}_{23}}{\partial x_{2}}+\frac{2 \delta}{B} \widetilde{S}_{23}
\end{array}\right) \\
& =\frac{\mu U_{0}}{r_{0}^{2}} \underbrace{\left(\begin{array}{l}
\frac{\partial \tau_{11}}{\partial x_{1}}+\frac{\partial \tau_{12}}{\partial x_{2}}+\frac{\delta}{B} \tau_{12} \\
\frac{\partial \tau_{12}}{\partial x_{1}}+\frac{\partial \tau_{22}}{\partial x_{2}}+\frac{\delta}{B}\left(\tau_{22}-\tau_{33}\right) \\
\frac{\partial \tau_{13}}{\partial x_{1}}+\frac{\partial \tau_{23}}{\partial x_{2}}+\frac{2 \delta}{B} \tau_{23}
\end{array}\right)}_{\nabla^{\star} \cdot\left(\boldsymbol{\tau}\left(D^{\star} \boldsymbol{u}\right)\right)},
\end{aligned}
$$

where we dropped the dependance on $\widetilde{D} \widetilde{\boldsymbol{u}}$ and $D^{\star} \boldsymbol{u}$. Taking into account these identities and substituting in equation $(2.1)_{1}$, we obtain

$$
\frac{\rho U_{0}^{2}}{r_{0}} \boldsymbol{u} \cdot \nabla^{\star} \boldsymbol{u}+\frac{\mu U_{0}}{r_{0}^{2}} \nabla^{\star} \pi=\frac{\mu U_{0}}{r_{0}^{2}} \nabla^{\star} \cdot\left(\boldsymbol{\tau}\left(D^{\star} \boldsymbol{u}\right)\right)
$$

which, by multiplying by $\frac{r_{0}^{2}}{\mu U_{0}}$, gives equation (2.7) $)_{1}$. Finally, by taking into account (A.4), we obtain

$$
\widetilde{\nabla} \cdot \widetilde{\boldsymbol{u}}=\frac{\partial \widetilde{u}_{1}}{\partial \widetilde{x}_{1}}+\frac{\partial \widetilde{u}_{2}}{\partial \widetilde{x}_{2}}+\frac{\widetilde{u}_{2}}{R B}=\frac{U_{0}}{r_{0}} \underbrace{\left(\frac{\partial u_{1}}{\partial x_{1}}+\frac{\partial u_{2}}{\partial x_{2}}+\frac{\delta}{B} u_{2}\right)}_{\nabla^{\star} \cdot \boldsymbol{u}},
$$

showing that $(2.1)_{2}$ implies $(2.7)_{2}$.

\section{References}

[1] C. Amrouche, V. Girault, Decomposition of vector spaces and application to the Stokes problem in arbitrary dimension. Czechoslovak Mathematical Journal, 44 (1994) 109-140.

[2] A. A. Berger, L. Talbot, L.-S. Yao, Flow in curved pipes, Ann. Rev. Fluid Mech., 15 (1983) $461-512$

[3] V. Coscia, A. M. Robertson, Existence and uniqueness of steady, fully developed flows of second order fluids in curved pipes, Math. Models Methods Appl. Sci. 6 (1) (2001) 1055-1071.

[4] P. Daskopoulos, A. M. Lenhoff, Flow in curved ducts: bifurcation structure for stationary ducts, J. Fluid Mech., 203 (1989) 125-148. 
[5] W. R. Dean, Note on the motion of fluid in curved pipe, Philos. Mag., 20 (1927) 208-223.

[6] W. R. Dean, The streamline motion of fluid in curved pipe, Philos. Mag., 30 (1928) 673-695.

[7] J. Eustice, Flow of water in curved pipes, Proc. R. Soc. Lond. A 84 (1910) 107-118.

[8] J. Eustice, Experiments of streamline motion in curved pipes, Proc. R. Soc. Lond. A 85 (1911) 119-131.

[9] Y. Fan, R. I. Tanner, N. Phan-Thien, Fully developed viscous and viscoelastic flows in curved pipes, J. Fluid Mech., 440 (2001) 327-357.

[10] G. P. Galdi, A. M. Robertson, On flow of a Navier-Stokes fluid in curved pipes. Part I: Steady flow, Applied Math. Letters, 18 (2005) 1116-1124.

[11] G. P. Galdi, An introduction to the mathematical theory of the Navier-Stokes equations, Vol. I and II, Springer Tracts in Natural Philosophy 38, 39, 2nd edition, Springer-Verlag, New York, 1998.

[12] H. Ito, Flow in curved pipes, JSME Int. J., 30 (1987) 543-552.

[13] W. Jitchote, A. M. Robertson, Flow of second order fluids in curved pipes, J. Non-Newtonian Fluid Mech. 90, 2000, 91-116.

[14] O. A. Ladyzhenskaya, New equations for the description of motion of viscous incompressible fluids and solvability in the large of boundary value problems for them, Proc. Stek. Inst. Math. 102 (1967) 95-118.

[15] O. A. Ladyzhenskaya, On some modifications of the Navier-Stokes equations for large gradients of Velocity, Zap. Naučn. Sem. Leningrad. Otdel. Mat. Inst. Steklov (LOMI) 7 (1968) 126-154.

[16] O. A. Ladyzhenskaya, The mathematical theory of viscous incompressible flow, Gordon and Beach, New York, 1969.

[17] J.-L. Lions, Quelques méthodes de résolution des problèmes aux limites non linéaires, Dunod, Gauthier-Villars, Paris, 1969.

[18] J. NeČAs, J. Málek, J. Rokyta, M. RuŽiČKa, Weak and measure-valued solutions to evolutionary partial differential equations, Applied Mathematics and Mathematical Computation, Vol. 13, Chapmann and Hall, London, 1996.

[19] A. M. Robertson, On viscous flow in curved pipes of non-uniform cross section, Inter. J. Numer. Meth. fluid., 22 (1996) 771-798.

[20] A. M. Robertson, S. J. Muller, Flow of Oldroyd-B fluids in curved pipes of circular and annular cross-section, Int. J. Non-Linear Mechanics, 31 (1996) 1-20.

[21] W. Y. Soh, S. A. Berger, Fully developed flow in a curved pipe of arbitrary curvature ratio, Int. J. Numer. Meth. Fluids, 7 (1987) 733-755.

[22] H. C. Topakoglu, M. A. Ebadian, On the steady laminar flow of an incompressible viscous fluid in a curved pipe of elliptical cross-section, J. Fluid Mech., 158 (1985) 329-340.

[23] Z. H. Yang, H. B. Keller, Multiple laminar flows through curved pipes, Appl. Numer. Math., 2, (1986) 257-271. 